NBER WORKING PAPER SERIES

\title{
PARALYZED BY FEAR: RIGID AND DISCRETE PRICING UNDER DEMAND UNCERTAINTY
}

\author{
Cosmin L. Ilut \\ Rosen Valchev \\ Nicolas Vincent \\ Working Paper 22490 \\ http://www.nber.org/papers/w22490
NATIONAL BUREAU OF ECONOMIC RESEARCH
1050 Massachusetts Avenue
Cambridge, MA 02138
August 2016

We would like to thank Etienne Gagnon, Han Hong, Nir Jaimovich, John Leahy, Virgiliu Midrigan, Krisztina Molnar, Martin Schneider and Luminita Stevens, as well as seminar and conference participants at the 9th Annual CIGS conference on Macroeconomic Theory and Policy, 22nd Annual Research Conference of the Dutch Central Bank, Ambiguity and Robustness in Macroeconomics and Finance Workshop, Ambiguity and its Implications in Finance and Macroeconomics Conference, Behavioral Aspects of Macroeconomics and Finance Conference, Boston Macro Juniors Workshop, Chicago Fed, CIREQ Montréal Macro conference, Duke, ECB, ESWC, NBER Summer Institute, NBER EFG meeting, Northwestern, SED, Stanford, UC San Diego, U Penn, UT Austin, UVA for helpful discussions and comments. Vincent acknowledges nancial support from the Fondation HEC Montréal. The views expressed herein are those of the authors and do not necessarily reflect the views of the National Bureau of Economic Research.

NBER working papers are circulated for discussion and comment purposes. They have not been peerreviewed or been subject to the review by the NBER Board of Directors that accompanies official NBER publications.

(C) 2016 by Cosmin L. Ilut, Rosen Valchev, and Nicolas Vincent. All rights reserved. Short sections of text, not to exceed two paragraphs, may be quoted without explicit permission provided that full credit, including $\odot$ notice, is given to the source. 
Paralyzed by Fear: Rigid and Discrete Pricing under Demand Uncertainty

Cosmin L. Ilut, Rosen Valchev, and Nicolas Vincent

NBER Working Paper No. 22490

August 2016, Revised in September 2020

JEL No. C1,D8,E3,L11

\begin{abstract}
$\underline{\text { ABSTRACT }}$
We propose a new theory of price rigidity based on firms' Knightian uncertainty about their competitive environment. This uncertainty has two key implications. First, firms learn about the shape of their demand function from past observations of quantities sold. This learning gives rise to kinks in the expected pro $t$ function at previously observed prices, making those prices both sticky and more likely to reoccur. Second, uncertainty about the relationship between aggregate and industry-level inflation generates nominal rigidity. We prove the main insights analytically and quantify the effects of our mechanism. Our estimated quantitative model is consistent with a wide range of micro-level pricing facts that are typically challenging to match jointly. It also implies significantly more persistent monetary non-neutrality than in standard models, allowing it to generate large real effects from nominal shocks.

Cosmin L. Ilut

Department of Economics

Duke University

223 Social Sciences Building

Box 90097

Durham, NC 27708

and NBER

cosmin.ilut@duke.edu

Rosen Valchev

Boston College

Department of Economics

Maloney Hall 396

140 Commonwealth Avenue

Chestnut Hill, MA 02467

US

rosen.valchev@bc.edu

Nicolas Vincent

HEC Montreal

Institute of Applied Economics

3000, chemin de la Côte-Sainte-Catherine

Montréal (Québec), H3T 2A7

Canada

nicolas.vincent@hec.ca
\end{abstract}




\section{Introduction}

Macroeconomists have long recognized that incomplete price adjustment plays a crucial role in the amplification and propagation of macroeconomic shocks. On the one hand, there is ample evidence that aggregate inflation responds only slowly to monetary shocks (e.g. Christiano et al. (2005)). On the other, numerous studies have shown that at the micro level, prices are not as sticky as the aggregates imply. They do, however, display other puzzling characteristics that could play a crucial macro role (e.g. Bils and Klenow (2004)).

In this paper, we propose a new theory of price rigidity based on firms' Knightian uncertainty about the demand for their product. This uncertainty endogenously generates an as-if kink in expected profits, and hence a first-order cost of moving away from a previously posted price. The mechanism not only leads to price stickiness, but also price memory and a number of additional micro-level pricing facts. These features allow it to generate significant monetary non-neutrality despite prices changing relatively frequently, as in the data.

Our economy is composed of a continuum of industries, each populated with monopolistic firms who face uncertainty about their competitive environment. In order to evaluate how demand changes as a function of the nominal price they post, firms need to jointly assess (i) the unknown demand curve, as a function of the relevant relative price; and (ii) the relative price itself, which equals the firm's nominal price minus the unobserved industry price index. Uncertainty about both jointly leads to nominal rigidity.

Standard models abstract from such uncertainty, typically assuming that firms know the structure of the economy and observe the price index of the competition. In contrast, we assume firms face specification doubts about the model of demand. We capture such doubts by drawing on the large experimental and theoretical work motivated by Ellsberg (1961) that distinguishes between risk (uncertainty with known odds) and ambiguity, or Knightian uncertainty (unknown odds). ${ }^{1}$ In particular, we model the aversion to ambiguity using the multiple priors preferences axiomatized by Gilboa and Schmeidler (1989), and characterize the firm's lack of confidence through a set of possible prior distributions over both the unknown demand shape and the unknown relative price.

To this end, we assume that the firm, similar to an econometrician, estimates its unknown demand function from past observations of prices and quantities sold. In doing so, the firm knows demand is a smooth, downward-sloping function, but is not confident (i) that it belongs to a particular parametric family of functions, and (ii) in a unique probability

\footnotetext{
${ }^{1}$ See Machina and Siniscalchi (2014) for a review of related theory and experiments. The latter confirm the basic conjecture in Ellsberg (1961) of prevalent aversion to ambiguity, and includes surveys and experiments specifically involving business managers, such as in Einhorn and Hogarth (1986), March and Shapira (1987), Kunreuther et al. (1993) and Maffioletti and Santoni (2005).
} 
measure over the space of potential demand functions. In particular, while the firm knows that its demand is the sum of a price-sensitive component and a temporary shock, it faces a signal extraction problem because it does not observe each separately. The firm uses its history of quantities sold at past prices, together with its set of priors, to form a set of conditional beliefs about its demand function.

The firm has two sources of information on the unknown industry-wide price level. The first are periodic marketing reviews that fully reveal its current value. The second is the aggregate price level, which the firm observes freely, but is an imperfect signal of the firm's specific industry price, because the link between industry and aggregate prices is uncertain and ambiguous - while the firm understands that the two indices are cointegrated in the long run, it is not confident about their short-run relationship. Specifically, over short horizons, observing a change in the aggregate price level does not convince the firm that the industry price has evolved in the same way. We model this lack of confidence as a set of potential relationships, resulting in a set of conditional beliefs about the current industry price given an observed value for the aggregate price level. ${ }^{2}$

In the face of ambiguity about both its demand function and its effective relative price, the firm optimally selects a nominal price as if nature draws the joint prior distribution that implies the lowest (i.e. worst-case) conditional expected demand. A key result is that this joint worst-case belief changes endogenously around the level of previously posted prices relative to the firm's best, unambiguous estimate of industry inflation. The reason is intuitive: an unambiguous price increase sets in motion a concern for a "double whammy" that nature draws (i) the most locally-elastic demand function allowed by the prior set and (ii) the largest decrease in the unobserved industry price given the relevant set of conditional beliefs. Hence, the firm fears the increase in its relative price is larger than expected and that demand is especially sensitive to it. The opposite concern occurs in the case of a decrease in price - the firm fears that demand is inelastic and the industry price index rose.

This endogenous switch in the worst-case scenario is at the heart of our mechanism: it generates kinks in expected demand and thus price rigidity. ${ }^{3}$ An unambiguous change in the relative price would move the firm away from the safety of previously accumulated information, and therefore expose it to increased uncertainty about the shape of demand.

When interacted with ambiguity about the industry price, and therefore uncertainty

\footnotetext{
${ }^{2}$ Using the BLS' most disaggregated 130 CPI indices as well as aggregate CPI, we present evidence that an econometrician would generally have very little confidence that short-run aggregate inflation is related to industry-level inflation, even though she can be confident that the two are cointegrated in the long-run.

${ }^{3}$ Such endogeneity is the defining feature of the Ellsberg experiment: when the agent evaluates a bet on either a black or a white ball from the ambiguous urn, he does so as if the probability of drawing that ball is less than 0.5 in either case. This behavior is inconsistent with any single probability measure on the associated state space, but can be explained by the multiple-priors model.
} 
about the relative price achieved by a specific choice of nominal price, the rigidity becomes nominal. The key is that the optimal choice robust to the joint uncertainty is to price as if short-run industry inflation is not forecastable, and thus keep nominal prices rigid to take advantage of the perceived kinks in demand. Intuitively, a directly observed change in the industry price index would lead to an immediate adjustment in the nominal price, since it has an unambiguous effect on the relative price. In contrast, the effect of aggregate inflation on the underlying industry price level is ambiguous: if the firm assumes a positive link and responds by increasing its nominal price, this would be precisely the wrong action in case the industry price actually fell, and vice versa if it were to act under the belief that the two are negatively correlated. These fears make aggregate (or other) indexation suboptimal.

In sum, a change in the relative price away from a previously observed value incurs an endogenous, time-varying cost in terms of expected profits, whose properties we derive analytically. First, this cost is locally first-order, so that a firm has an incentive to keep its estimated relative price constant even when hit with marginal-cost shocks. Second, conditional on changing, the firm is inclined to repeat a price it has already posted in the past - such previously estimated relative prices are associated with kinks in expected profits, and become 'reference' price points. Third, the cost is perceived to be larger for prices that have been observed more often in the past, as higher signal-to-noise ratios deepen the kinks. Fourth, given the resulting time-variation in the first-order cost, the firm may find it optimal to implement small or large price changes. Fifth, the perceived cost of changing a posted price increases with the value of the demand shock at that price. Sixth, even though firms are forward-looking, the optimal experimentation strategy may in fact reinforce stickiness.

Since the worst-case belief is that aggregate inflation is uninformative about industry prices, it follows that between marketing review periods, the firm faces a first-order cost of nominal adjustment with similar properties. This results in what looks like "price plans", where the price series tends to bounce around just a few repeated price points. One important difference with standard "price plan" models is that in our framework, the endogenous price plan evolves gradually over time, incorporating new prices one-by-one as the firm experiments and learns about demand at new price points. We document that this novel implication of gradual adjustment in price plans is prevalent in the data, and also show that it has important implications for the aggregate transmission of monetary shocks in the model.

In addition to the analytical results, we evaluate the model quantitatively. We solve numerically for its stochastic steady state and estimate the parameters by targeting standard micro-level pricing moments from the IRI Academic Dataset. We then show that our learning mechanism is quantitatively consistent with a rich set of additional moments that are typically considered challenging to match jointly: (i) memory in prices; (ii) co-existence 
of small and large price changes; (iii) pricing behavior over the product's life-cycle; (iv) downward-sloping hazard function of price changes; as well as a novel implication that (v) a price with a positive demand innovation is less likely to change. ${ }^{4}$

Lastly, we show that our quantitative model predicts large and persistent real effects from a nominal spending shock. These effects occur even though the model is consistent with the observed high frequency and large median absolute size of price changes, typically taken to imply low monetary non-neutrality in standard state-dependent models due to the Golosov and Lucas (2007) selection effect. The reason lies in the endogenous memory of prices, and in particular the slow adjustment of the effective "price plan", an empirically supported feature unique to our model. Because it significantly slows down the transmission of nominal shocks, this type of memory delivers more persistent real effects than in standard price-plan models. As a result, our mechanism has important novel features that can arguably help fit the evidence of persistent monetary policy effects (Christiano et al. (2005)).

Next, we review the literature. Section 2 derives analytical results in a real model, while Section 3 expands them to a nominal model. Section 4 quantifies the mechanism.

\section{Relation to the literature}

By connecting learning under ambiguity to the problem of a firm setting prices, our paper relates to multiple strands of the literature. First is the extensive body of work on theories of real and nominal price rigidity. With respect to the former, it relates to work on kinked demand curves, including Stigler (1947), Stiglitz (1979), Ball and Romer (1990), Kimball (1995) and Dupraz (2016). While in these models the kinks are a feature of the true demand curve, in our setup they arise only as a result of uncertainty about the shape of demand, and an econometrician would not be expected to find evidence of actual kinks.

On nominal rigidity, we connect to the literature that emphasizes the role of imperfect information in generating slow adjustment to aggregate nominal shocks, including Mankiw and Reis (2002), Sims (2003), Woodford (2003), Reis (2006) and Mackowiak and Wiederholt (2009). However, while in order to obtain fully rigid prices these models typically require additional frictions, (e.g. a menu cost), we show that uncertainty alone can lead to inaction. ${ }^{5}$

In testing our mechanism against a rich set of overidentifying restrictions, we connect to

\footnotetext{
${ }^{4}$ Given the importance of controlling for unobserved heterogeneity in recovering the hazard function facts, and the novelty of the role of demand signals for pricing decisions, our detailed documentations of these two particular conditional moments is of independent empirical interest for the pricing literature.

${ }^{5}$ Bonomo and Carvalho (2004) and Knotek and Edward (2010) are early examples of merging information frictions with a physical cost or an exogenous probability of price adjustment. Recent models of rational inattention (e.g. Woodford (2009) or Stevens (2014)) assume that memory, including assessing the passage of time, is costly. Therefore, in periods when the firm is inattentive, it does not index to aggregate inflation.
} 
several literatures on pricing models that have grappled with one or more of these facts. ${ }^{6}$

First, in our model, prices tend to return to previous values, giving rise to discreteness and memory. This empirical regularity has been well documented following the seminal work of Eichenbaum et al. (2011), as it presents a challenge to standard state-dependent pricing theories that rely on a single fixed cost of a price change. To address this, the literature has used exogenously defined price plans (Eichenbaum et al. (2011)), heterogeneous menu costs (Kehoe and Midrigan (2015)), and rational inattention, emphasizing the discrete nature of the optimal signal structure under certain conditions (Matějka (2015) and Stevens (2014)). As discussed in the introduction, our mechanism differs from these frameworks both in terms of its micro-foundations, testable predictions and aggregate implications.

The second set of related models is on pricing under demand uncertainty. The standard approach has been to analyze learning about a parametric demand curve under expected utility. ${ }^{7}$ Unlike our environment, this does not result in kinks in conditional beliefs at old prices, and thus price stickiness and memory. In fact, the objective in introducing learning in existing models has not been to generate stickiness, but instead to match other facts, such as the shape of hazard function (Bachmann and Moscarini (2011), Baley and Blanco (2018)) or the pricing behavior over the product life cycle (Argente and Yeh (2017)). Our model also matches these facts, in addition to others such as stickiness and memory.

At its core, our framework fits within the literature motivated by the classic work of Ellsberg (1961), such as Gilboa and Schmeidler (1989), Dow and Werlang (1992), and Epstein and Schneider (2003). In the field of industrial organization, Bergemann and Schlag (2011) studies a static pricing problem with multiple priors over the distribution of buyers' valuations, while Handel and Misra (2015) extends that analysis to a two-period model with maxmin regret that allows for consumer heterogeneity. In contrast, we simplify the consumer's side of the market and instead develop a tractable learning environment to study how the accumulation of information about a set of demand curves leads to pricing behavior that is empirically supported and of interest for macroeconomic models.

\section{Analytical Model}

In this section, we develop the key insights of our mechanism in the context of a simple, analytically-tractable model that does not distinguish between real and nominal prices. We present the full nominal model in Section 3.

\footnotetext{
${ }^{6}$ In this, we follow the spirit of a broad literature that documents micro-level facts aimed at disciplining theoretical models of rigidity, such as Bils and Klenow (2004), Klenow and Kryvtsov (2008), Nakamura and Steinsson (2008), Klenow and Malin (2010) and Campbell and Eden (2014), among many.

${ }^{7}$ An early contribution is Rothschild (1974), who frames the learning process as a two-arm bandit problem.
} 
We study a monopolistic firm that each period sells a single good, facing the log demand

$$
y\left(p_{t}\right)=x\left(p_{t}\right)+z_{t},
$$

where $p_{t}$ is the log price. Demand consists of two components - the price-sensitive $x\left(p_{t}\right)$ and a price-insensitive component captured by $z_{t}$. The firm's time- $t$ realized profit is:

$$
v_{t}=\left(e^{p_{t}}-e^{c_{t}}\right) e^{y\left(p_{t}\right)}
$$

where we have assumed a linear cost function, with $c_{t}$ denoting the time- $t$ log marginal cost.

The decomposition of demand in (1) serves two purposes. First, it generates a motive for signal extraction. In this respect we assume that the firm only observes total quantity sold, $y\left(p_{t}\right)$, but not the underlying $x\left(p_{t}\right)$ and $z_{t}$ separately. Furthermore, we model $z_{t}$ as iid, and thus past demand realizations serve as noisy signals about the unknown function $x(p)$.

The second purpose is to differentiate between risk and ambiguity. We model $z_{t}$ as purely risky, and give the firm full confidence that it is iid and drawn from the known Gaussian distribution $z_{t} \sim N\left(0, \sigma_{z}^{2}\right)$. On the other hand, the $x\left(p_{t}\right)$ component is ambiguous, meaning that the firm is not fully confident in the distribution from which it has been drawn and does not have a unique prior over it.

Instead, the firm entertains a whole set of possible priors, $\Upsilon_{0}$, which is not restricted to a given parametric family. Each individual prior in the set $\Upsilon_{0}$ is a Gaussian Process distribution, $G P\left(m(p), K\left(p, p_{t}\right)\right)$, with mean function $m(p)$ and covariance function $K\left(p, p_{t}\right)$. A Gaussian Process distribution is the generalization of the Gaussian distribution to infinitesized collections of real-valued random variables, and is thus a convenient choice of a prior for doing Bayesian inference on function spaces. It has the defining feature that for any finite sub-collection of function inputs, e.g. a vector of prices $\mathbf{p}=\left[p_{1}, \ldots, p_{N}\right]^{\prime}$ for some $N>1$, the corresponding vector of quantities demanded $x(\mathbf{p})$ is distributed as

$$
x(\mathbf{p}) \sim N\left(\left[\begin{array}{c}
m\left(p_{1}\right) \\
\vdots \\
m\left(p_{N}\right)
\end{array}\right],\left[\begin{array}{ccc}
K\left(p_{1}, p_{1}\right) & \ldots & K\left(p_{1}, p_{N}\right) \\
\vdots & \ddots & \vdots \\
K\left(p_{N}, p_{1}\right) & \ldots & K\left(p_{N}, p_{N}\right)
\end{array}\right]\right)
$$

where the mean function $m(p)$ controls the average slope of the underlying functions $x(p)$, and the covariance function $K\left(p, p^{\prime}\right)$ controls their smoothness. In other words, this distribution is a cloud of functions dispersed around $m(p)$, according to the covariance function $K\left(p, p^{\prime}\right)$.

We model ambiguity by assuming that all priors have the same covariance function, but different mean functions. We assume that the covariance function is of the widely-used 
squared exponential class (see Rasmussen and Williams (2006)):

$$
K\left(p, p^{\prime}\right)=\operatorname{Cov}\left(x(p), x\left(p^{\prime}\right)\right)=\sigma_{x}^{2} e^{-\psi\left(p-p^{\prime}\right)^{2}} .
$$

The function has two parameters: $\sigma_{x}^{2}$ measures the prior variance about demand at any given price, and $\psi>0$ controls the extent to which information about demand at some price $p$ is informative about its value at a different price $p^{\prime}$. The larger is $\psi$, the faster the correlation between quantity demanded at different prices declines with the distance between those prices. $^{8}$ This covariance function parsimoniously, yet flexibly, captures the natural prior view that there is an imperfect and declining correlation between demand at different prices. Additionally, this prior puts zero probability on demand functions that are not infinitely differentiable - thus any non-differentiability in the firm's eventual worst-case perceptions about demand are fully attributable to the ambiguity-aversion mechanism.

The multiple priors differ in their mean function $m(p)$. We assume that the set of entertained $m(p)$ is centered around the true DGP of a standard log-linear demand function, $x^{D G P}(p)=-b p$, so that the potential $m(p)$ lie within an interval of width $2 \gamma$ around $x^{D G P}(p)$,

$$
m(p) \in[-\gamma-b p, \gamma-b p]
$$

The parameter $\gamma>0$ controls the size of perceived ambiguity and captures the firm's lack of confidence in assigning probability assessments over the mean demand at a given price $p$.

In addition, to preclude any ex ante built-in non-differentiability, we also bound the local variability of admissible $m(p)$. The firm only entertains differentiable $m(p)$ functions with a derivative that lies within an interval centered around the derivative of the true DGP,

$$
m^{\prime}(p) \in[-b-\delta,-b+\delta]
$$

with $\delta>0$ controlling the size of that interval. Throughout we assume that $\delta \leq b$, hence the firm is at least confident that demand is weakly downward-sloping. As an illustration, the left panel of Figure 1 provides some examples of admissible demand schedules $m(p)$, out of the infinite set of functions that satisfy (3) and (4). Later, we explain how an ambiguity-averse firm extracts out of this set the kinked worst-case prior shown in the right panel.

The overall interpretation of our setup is that the firm has some a priori information on the true demand, but is not confident in a single probabilistic weighting of the potential

\footnotetext{
${ }^{8} \mathrm{~A}$ Gaussian Process with a higher $\psi$ has a higher rate of change (i.e. larger derivative) and its value is more likely to experience a bigger change for the same change in $p$. For example, it can be shown that the mean number of zero-crossings over a unit interval is given by $\frac{\psi}{\sqrt{2} \pi}$.
} 


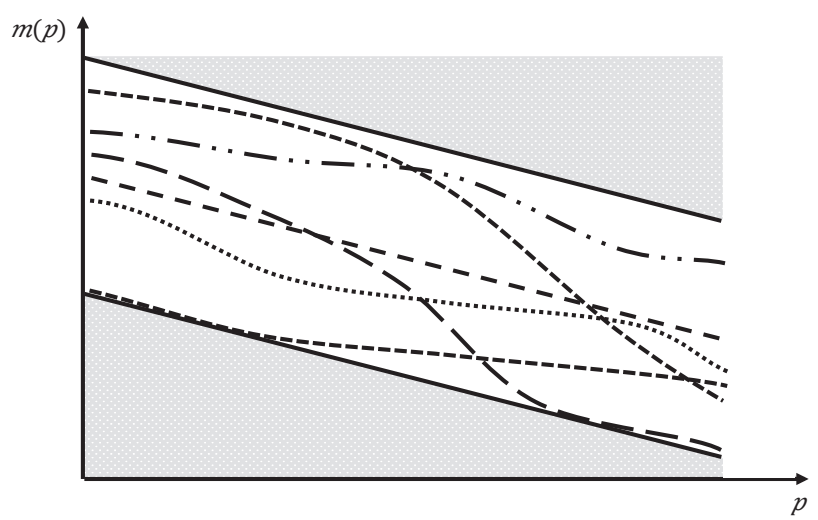

(a) Set of priors

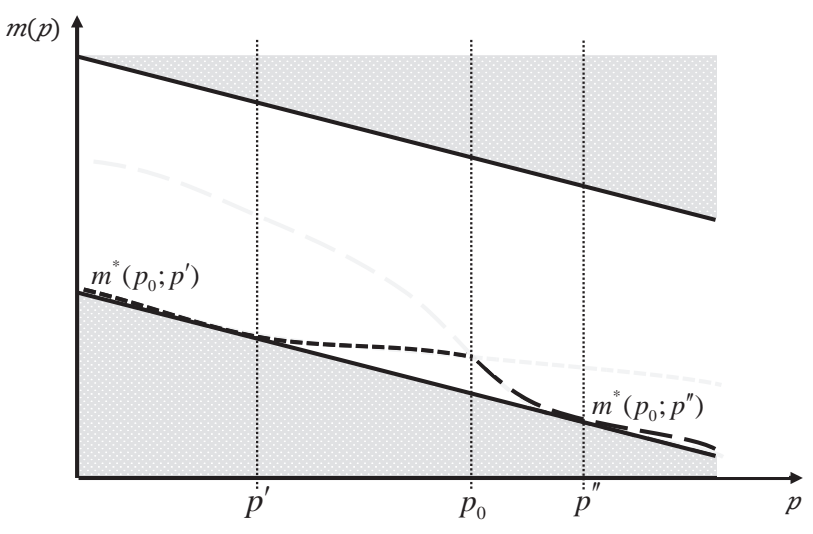

(b) Worst-case prior

Figure 1. Illustrative set of priors and worst-case prior

demand schedules (i.e. a single prior), nor is it able to restrict attention to a particular parametric family of demand functions. The set of admissible beliefs may itself reflect the disagreement between heterogeneous, but otherwise unique, prior beliefs expressed by various agents inside the firm. The agent that takes the pricing decisions is not confident how to probabilistically weigh them as these beliefs are all entertained as reasonable priors. ${ }^{9}$

The parametrization of ambiguity characterizing the sets (3) and (4) serves two purposes. First, it avoids overparameterizing $\Upsilon_{0}$, so that we represent the ambiguity over a nonparametric family of functions using only two parameters, $\gamma$ and $\delta$. Second, it contains the minimal ingredients necessary for our main results. In particular, when $\gamma=0$, the set $\Upsilon_{0}$ collapses to a singleton, hence the firm has a unique prior and there is no ambiguity. On the other hand, with $\delta=0$ the firm faces no ambiguity about the shape of the demand function, which is the key ingredient of our theory. Uncertainty about the local elasticity of demand (i.e. $\delta>0$ ) is at the heart of our mechanism.

\subsection{Information and Preferences}

The timing of choices and revelation of information is as follows: We assume that $c_{t}$ is known at the end of $t-1$ and that it is a continuous random variable following a Markov process with a conditional density function $g\left(c_{t} \mid c_{t-1}\right)$. The firm enters period $t$ with information on the history of all previously-sold quantities $y^{t-1}=\left[y\left(p_{1}\right), \ldots, y\left(p_{t-1}\right)\right]^{\prime}$ and the corresponding prices at which those were observed $p^{t-1}=\left[p_{1}, \ldots, p_{t-1}\right]^{\prime}$, where a superscript denotes history

\footnotetext{
${ }^{9}$ The connection between the set of beliefs about $m(p)$ to the dispersion of prior forecasts made by experts inside a firm allows us to empirically discipline the magnitude of ambiguity in the model's quantitative evaluation. The view that uncertainty is not primarily a probabilistic concept is consistent with the survey and experimental evidence involving business managers (see for example March and Shapira (1987)).
} 
up to that time. The firm updates its beliefs about demand conditional on $\varepsilon^{t-1}=\left\{y^{t-1}, p^{t-1}\right\}$, observes $c_{t}$ and posts a price $p_{t}$ that maximizes expected profits, as detailed further below. At the end of period $t$, the idiosyncratic demand shock $z_{t}$ is realized and the firm updates its information set with the resulting quantity sold $y\left(p_{t}\right)$, and the new cost $c_{t+1}$.

The firm uses the available data $\varepsilon^{t-1}$ to update the set of initial priors $\Upsilon_{0}$. Learning occurs through standard Bayesian updating, prior-by-prior - for each prior in the initial set $\Upsilon_{0}$, the firm uses the new information and Bayes' Rule to obtain a posterior distribution. Given that there is a set of priors, the Bayesian update results in a set of posteriors. In particular, we denote by $x_{t-1}(p ; m(p))$ the posterior Gaussian distribution of $x(p)$, conditional on $\varepsilon^{t-1}$ and a particular prior $m(p)$. We denote the conditional mean and variance of demand as:

$$
\begin{aligned}
\widehat{x}_{t-1}\left(p_{t} ; m(p)\right) & :=E\left[x(p) \mid \varepsilon^{t-1} ; m(p)\right] \\
\widehat{\sigma}_{t-1}^{2}(p) & :=\operatorname{Var}\left[x(p) \mid \varepsilon^{t-1}\right] .
\end{aligned}
$$

While the conditional expectation depends on the prior $m(p)$, the variance is the same for all priors, as they differ only in their means. The evolution of beliefs is analytically tractable and follows the standard Bayesian-updating formulas derived in Online Appendix A.1.

The firm is owned by an agent who is ambiguity averse and has recursive multiple priors utility (Epstein and Schneider (2003)), so that she values the firm's profits as:

$$
V\left(\varepsilon^{t-1}, c_{t}\right)=\max _{p_{t}} \min _{m(p) \in \Upsilon_{0}} E\left[v\left(\varepsilon_{t}, c_{t}\right)+\beta V\left(\varepsilon^{t}, c_{t+1}\right) \mid \varepsilon^{t-1}, c_{t}\right]
$$

where $v\left(\varepsilon_{t}, c_{t}\right)$ is the per-period profit defined in (2). The firm forms its conditional beliefs and evaluates the expected profits and continuation utility using the available information $\varepsilon^{t-1}$ and the prior $m^{*}\left(p ; p_{t}\right)$ that achieves the worst-case belief, given a pricing choice $p_{t}$.

Importantly, the minimization over the priors is conditional on the choice of $p_{t}$. We conjecture and verify that the minimizing prior $m^{*}\left(p ; p_{t}\right)$ is such that, for a given price $p_{t}$ and history $\varepsilon^{t-1}$, it implies the lowest admissible expected demand $\widehat{x}_{t-1}\left(p_{t} ; m^{*}\left(p ; p_{t}\right)\right)$ at that price. Thus, for any price $p_{t}$ the firm worries that, given the data it has seen, the underlying demand is low and hence maximizes over $p_{t}$ under the worst-case belief $\widehat{x}_{t-1}\left(p_{t} ; m^{*}\left(p ; p_{t}\right)\right)$.

\subsection{As-if kinks in demand from learning}

To gain intuition on how updating and the basic mechanism work, we start by considering the simplest case, where the information set $\varepsilon^{t-1}$ contains only observations of demand at a single price point $p_{0}$ that has been seen $N_{0}$ times, and has an associated average demand realization $\bar{y}_{0}=x\left(p_{0}\right)+\frac{1}{N_{0}} \sum_{i=1}^{N_{0}} z_{i}$. For a given prior $m(p)$, the joint distribution of the 
signal and the unknown demand function $x$ at any price $p$ is:

$$
\left[\begin{array}{c}
x(p) \\
\bar{y}_{0}
\end{array}\right] \sim N\left(\left[\begin{array}{c}
m(p) \\
m\left(p_{0}\right)
\end{array}\right],\left[\begin{array}{cc}
\sigma_{x}^{2} & \sigma_{x}^{2} e^{-\psi\left(p-p_{0}\right)^{2}} \\
\sigma_{x}^{2} e^{-\psi\left(p-p_{0}\right)^{2}} & \sigma_{x}^{2}+\sigma_{z}^{2} / N_{0}
\end{array}\right]\right) .
$$

The distribution of $x(p)$ conditional on $\bar{y}_{0}$ is also Gaussian, and its expectation and variance are given by the familiar prior plus signal-updating formulas:

$$
\begin{aligned}
E\left(x(p) \mid \bar{y}_{0}, m(p)\right) & =m(p)+\alpha_{t-1}(p)\left[\bar{y}_{0}-m\left(p_{0}\right)\right] \\
\operatorname{Var}\left(x(p) \mid \bar{y}_{0}\right) & =\sigma_{x}^{2}\left(1-\alpha_{t-1}(p)\right)
\end{aligned}
$$

where the signal-to-noise ratio used to update beliefs of demand at a given price $p$ is

$$
\alpha_{t-1}(p)=\frac{\sigma_{x}^{2}}{\sigma_{x}^{2}+\sigma_{z}^{2} / N_{0}} e^{-\psi\left(p-p_{0}\right)^{2}}
$$

Thus, the Bayesian update of the conditional expectation in equation (6) combines the prior for demand at that price, $m(p)$, with the information revealed by the difference between the observed signal realization $\bar{y}_{0}$, and the prior expected demand at that price, $m\left(p_{0}\right)$. Also note that with $\psi>0$, the signal-to-noise ratio $\alpha_{t-1}(p)$ and the resulting reduction in uncertainty is largest right at the observed price $p_{0}$ : as the correlation of quantity demanded at different prices decreases with the distance between them, the information obtained from the signal at $p_{0}$ is most useful in updating the firm's beliefs about demand around that price.

\section{Worst-case prior}

The firm minimizes the conditional expectation of demand over the priors $m(p) \in \Upsilon_{0}$. The resulting worst-case prior $m^{*}\left(p ; p_{t}\right)$ depends on the price $p_{t}$ at which the firm computes its expected demand. From equation (6) we see that the conditional expectation of demand at $p_{t}=p_{0}$ is decreasing in $m\left(p_{0}\right)$, since $\alpha_{t-1}(p) \in(0,1)$. Hence, the worst-case belief corresponds to the prior with the lowest value of $m\left(p_{0}\right)$, so $m^{*}\left(p_{0} ; p_{t}\right)=-\gamma-b p_{0}$ by equation (3).

When updating demand at a price $p_{t} \neq p_{0}$, the firm minimizes over $m\left(p_{t}\right)$ and $m\left(p_{0}\right)$, as both appear in the updating equation. It is useful to re-write equation (6) as

$$
E\left(x\left(p_{t}\right) \mid \bar{y}_{0}, m(p)\right)=\underbrace{\left(1-\alpha_{t-1}\left(p_{t}\right)\right) m\left(p_{t}\right)}_{\text {Prior demand at } p_{t}}+\underbrace{\alpha_{t-1}\left(p_{t}\right)\left(\bar{y}_{0}+m\left(p_{t}\right)-m\left(p_{0}\right)\right)}_{\text {Signal at } p_{0}+\Delta \text { in Demand between } p_{t} \text { and } p_{0}},
$$

since it makes clear that uncertainty over the prior $m(p)$ affects both the overall level of expected demand (through the first term), and how the firm interprets its signal $\bar{y}_{0}$ (second 
term). The uncertainty about the shape of the demand function implies a lack of confidence in how information about demand at $p_{0}$ translates into information about the quantity demanded at $p_{t}$. Consequently, the firm minimizes over both the prior level of demand at $p_{t}$ and its likely change between $p_{t}$ and $p_{0}$, the position of the observed signal.

First, minimizing over the prior at the entertained price, $m\left(p_{t}\right)$, is straightforward - the worst-case is that it lies at the lower bound of the set $\Upsilon_{0}$, so that

$$
m^{*}\left(p_{t} ; p_{t}\right)=-\gamma-b p_{t}
$$

Second, the firm is worried that demand changes for the worse as the price moves from $p_{0}$ to $p_{t}$, implying a low value of $m\left(p_{t}\right)-m\left(p_{0}\right)$. Thus, the worst-case for $m\left(p_{0}\right)$ is to be as high as possible given the constraints on the level and derivatives of the admissible $m(p)$ and the worst-case level for $m\left(p_{t}\right)$. Crucially, this implies a switch in the worst-case demand shape between $p_{t}$ and $p_{0}$, depending on whether the firm considers a price increase or a decrease.

Conditional on a price increase, i.e. $p_{t}>p_{0}$, the worst-case is that demand is elastic, since this generates a larger drop in demand. The drop from $m\left(p_{0}\right)$ to $m\left(p_{t}\right)$ is disciplined by the constraints on $\Upsilon_{0}$, which restrict both the derivative of $m(p)$ at any price $p$, and the maximal level of $m\left(p_{0}\right)$. Therefore, the worst-case prior for $m\left(p_{0}\right)$ when $p_{t}>p_{0}$ is

$$
m^{*}\left(p_{0} ; p_{t}\right)=\min \left[\gamma-b p_{0},-\gamma-b p_{t}+(b+\delta)\left(p_{t}-p_{0}\right)\right]
$$

On the other hand, when the firm considers a price cut, i.e. $p_{t}<p_{0}$, it worries that demand is inelastic and that the price decrease generates as small of an increase in demand as possible. The worst-case is again restricted by the constraints on $\Upsilon_{0}$, and in particular the lower bound on the admissible derivative of demand in (4). Effectively, the firm worries demand is flat to the left of $p_{0}$, hence, the worst-case $m^{*}\left(p_{0} ; p_{t}\right)$ in this case is

$$
m^{*}\left(p_{0} ; p_{t}\right)=\min \left[\gamma-b p_{0},-\gamma-b p_{t}+(b-\delta)\left(p_{t}-p_{0}\right)\right]
$$

\section{Worst-case conditional expectation and kinks}

Having characterized the worst-case prior, we can now plug it in equation (6) to obtain the worst-case conditional expectation at any entertained price $p_{t}$. Since the worst-case prior changes depending on whether $p_{t}$ is above or below $p_{0}$, the conditional expectation $\widehat{x}_{t-1}^{*}\left(p_{t}\right) \equiv E\left(x\left(p_{t}\right) \mid q_{0}, m^{*}\left(p ; p_{t}\right)\right)$ equals the following piecewise function

$$
\widehat{x}_{t-1}^{*}\left(p_{t}\right)= \begin{cases}-\gamma-b p_{t}+\alpha_{t-1}\left(p_{t}\right)\left[\bar{y}_{0}-\left(-\gamma-b p_{0}\right)\right]-\alpha_{t-1}\left(p_{t}\right) \delta\left|p_{t}-p_{0}\right| & \text { if } p_{t} \in[\underline{p}, \bar{p}] \\ -\gamma-b p_{t}+\alpha_{t-1}\left(p_{t}\right)\left[\bar{y}_{0}-\left(\gamma-b p_{0}\right)\right] & \text { if } p_{t} \notin[\underline{p}, \bar{p}]\end{cases}
$$


where $\underline{p}=p_{0}-\frac{2 \gamma}{\delta}$ and $\bar{p}=p_{0}+\frac{2 \gamma}{\delta}$. For prices $p_{t} \in[\underline{p}, \bar{p}]$, the worst-case prior demand at $p_{0}$ is obtained by moving away from $m^{*}\left(p_{t} ; p_{t}\right)=-\gamma-b p_{t}$ along the steepest (flattest) possible demand curve, when $p_{t}$ is higher (lower) than $p_{0}$. At the threshold prices $\underline{p}, \bar{p}$, moving along these worst-case elasticities intersects the upper bound of the set $\Upsilon_{0}$, so the solution to the worst-case prior in equations (8) and (9) for prices $p_{t}$ outside $[p, \bar{p}]$ is given by $\gamma-b p_{0}$.

Thus, the multiple priors endogenously generate a kink in expected demand at $p_{0}$, as captured by the absolute value term $\left|p_{t}-p_{0}\right|$ in (10). In essence, the overall worst-case expectation is the result of splicing two different priors together - an elastic one when evaluating prices to the right of $p_{0}$, and an inelastic one to its left - which creates a kink, even though all individual priors are differentiable. Going back to Figure 1, panel (b) illustrates this splicing when entertaining setting some $p^{\prime}<p_{0}$ or $p^{\prime \prime}>p_{0}$, conditional on seeing a signal equal to the true DGP at a single price point $p_{0}$ and facing the set of priors in panel (a).

Putting everything together, the left panel of Figure 2 shows the resulting worst-case expected demand at any price $p_{t}$ (i.e. it plots equation (10)). Extending these derivations to the case where $\varepsilon^{t-1}$ contains observations at more than one price point is straightforward - Online Appendix A.1 describes the general formulas and an analytical approach to finding the worst-case prior. The intuition is the same as for the case of a single previously observed price: the worst-case is to set the prior at the entertained $p_{t}$ equal to the lowest bound of $\Upsilon_{0}$, and the level of the prior at the prices in $\varepsilon^{t-1}$ as high as admissible, given the restrictions on $\Upsilon_{0}$. The main difference is that because the endogenous switch in the worst-case priors now applies more generally at all previously-observed prices, the firm perceives kinks at all of them. To illustrate, panel (b) of Figure 2 plots the worst-case expectation when the firm has observed demand signals at two distinct price points $p_{0}$ and $p_{1}$, and naturally the worst-case expectation is kinked around both of these prices.

\subsection{An as-if cost of changing the price}

When choosing its price to maximize expected profits under the worst-case beliefs, the problem of the firm is dynamic: posting a price today affects not only current profits, but also next period's information set. Solving the full infinite horizon optimization problem is difficult numerically, because the size of the state space is unbounded, and explodes as the number of posted prices increases over time. For this reason, we split our analysis in three parts. In this section, we analyze a myopic problem that ignores the continuation value of information, but provides a tight analytical characterization of the first-order forces at play. Then in Section 2.4 we provide analytical results for a tractable approximation to the forward-looking problem, before numerically analyzing it extensively in Section 4. 


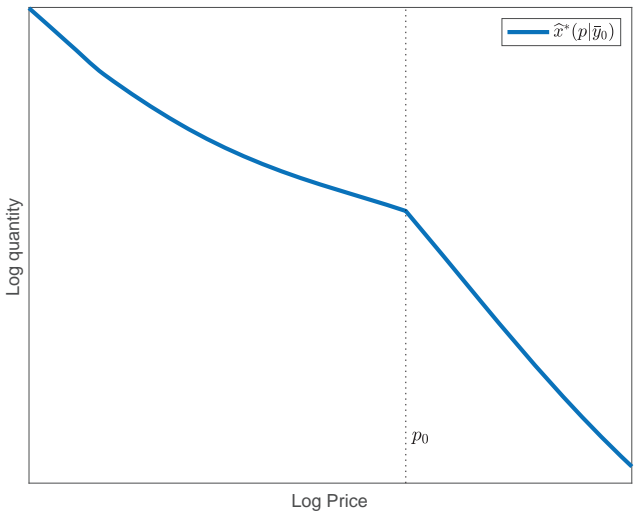

(a) One previously-observed price

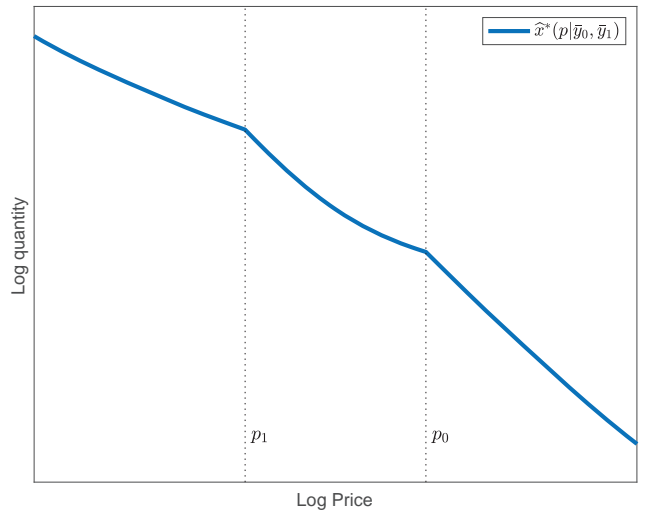

(b) Two previously-observed prices

Figure 2. Worst-case Expected Demand

A myopic firm chooses $p_{t}$ to maximize time-t's worst-case expected profit

$$
\max _{p_{t}} \min _{m(p) \in \Upsilon_{0}} E\left[v\left(\varepsilon_{t}, c_{t}\right) \mid \varepsilon^{t-1}, c_{t}\right]=\max _{p_{t}} \underbrace{\left(e^{p_{t}}-e^{c_{t}}\right) e^{\widehat{x}_{t-1}\left(p_{t} ; m^{*}\left(p ; p_{t}\right)\right)+.5 \widehat{\sigma}_{t-1}^{2}\left(p_{t}\right)+.5 \sigma_{z}^{2}}}_{=\nu^{*}\left(\varepsilon^{t-1}, c_{t}, p_{t}\right)} .
$$

The optimal behavior crucially hinges on the history of observations $\varepsilon^{t-1}$, which is an endogenous object, as it depends on the past actions of the firm. In order to describe analytically the key mechanics of the model, in this section we take $\varepsilon^{t-1}$ as given. We expand the analysis to the case where $\varepsilon^{t-1}$ is endogenous in Section 4 .

We start with the simplest case for the firm's information set and assume $\varepsilon^{t-1}$ contains a single price $p_{0}$, observed for $N_{0}$ number of times with an average quantity sold of $\bar{y}_{0}$. As shown before, this results in a kink in the as-if expected demand, and in turn this provides the firm with an incentive to keep its price rigid even when faced with variations in costs. To show this insight analytically, in Proposition 1 we consider a log-linear approximation of expected profits around $p_{0}$, which reveals a first-order loss of moving away from $p_{0}$.

Proposition 1. Define $\delta^{*}=\delta \operatorname{sgn}\left(p_{t}-p_{0}\right)$. For a given realization of $c_{t}$, the difference in worst-case expected profits at $p_{t}$ and $p_{0}$, up to a first-order approximation around $p_{0}$, is

$$
\ln v^{*}\left(\varepsilon^{t-1}, c_{t}, p_{t}\right)-\ln v_{0}^{*}\left(\varepsilon^{t-1}, c_{t}, p_{0}\right) \approx\left[\frac{e^{p_{0}}}{e^{p_{0}}-e^{c_{t}}}-\left(b+\alpha_{t-1}\left(p_{0}\right) \delta^{*}\right)\right]\left(p_{t}-p_{0}\right) .
$$

Proof. The switch in sign of $\delta^{*}$ follows from the worst-case expected demand in (10). Also, the marginal effect $\frac{\partial \alpha_{t-1}\left(p_{t}\right)}{\partial p_{t}}=0$ at $p_{t}=p_{0}$. For details, see Online Appendix A.2. 
Proposition 1 shows the locally-evaluated tradeoff of moving the price away from $p_{0}$. The first term in the squared brackets is the direct effect of a change in price, holding demand constant. The second term is the demand effect of a price change, by moving along the perceived demand elasticity. The fact that the elasticity switches by $\alpha_{t-1}\left(p_{0}\right) \delta^{*}$ around $p_{0}$, as indicated by the signum function, is the key mechanism in our model.

We now describe the main results that stem from this property.

\section{Result \#1: There exists an inaction region around previously-posted prices}

Given the first-order loss arising from the switch in elasticity around $p_{0}$, a direct implication (as derived explicitly in Corollary 1) is that there is a positive interval of $c_{t}$ realizations, around $c_{0}^{*} \equiv p_{0}-\ln \left(\frac{b}{b-1}\right)$, for which the firm keeps its current price fixed at $p_{t}=p_{0}$.

Corollary 1. Under the approximation in Proposition 1, $p_{0}$ is a local maximizer for any $c_{t} \in$ $\left(\underline{c}_{t-1,0}, \bar{c}_{t-1,0}\right)$, where $\underline{c}_{t-1,0}=c_{0}^{*}+\ln \left[\frac{b}{b-1} \frac{b-\alpha_{t-1}\left(p_{0}\right) \delta-1}{b-\alpha_{t-1}\left(p_{0}\right) \delta}\right]$ and $\bar{c}_{t-1,0}=c_{0}^{*}+\ln \left[\frac{b}{b-1} \frac{b+\alpha_{t-1}\left(p_{0}\right) \delta-1}{b+\alpha_{t-1}\left(p_{0}\right) \delta}\right]$.

Proof. For any $c_{t} \in\left(\underline{c}_{t-1,0}, \bar{c}_{t-1,0}\right)$ we have $\frac{e^{p_{0}}}{e^{p_{0}-e^{c_{t}}}} \in\left(b-\alpha_{t-1}\left(p_{0}\right) \delta, b+\alpha_{t-1}\left(p_{0}\right) \delta\right)$. Thus, the derivative in Proposition 1 is negative for $p_{t}>p_{0}$ when $\delta^{*}=\delta$, and positive for $p_{t}<p_{0}$ when $\delta^{*}=-\delta$. This gives the necessary and sufficient conditions for $p_{0}$ to be a local maximizer.

To gain intuition, consider an increase in cost to some $c_{t}>c_{0}^{*}$. This lowers the markup if the price remains at $p_{0}$, which gives the firm a reason to consider an increase in the price. However, when the firm entertains a higher price $p_{t}>p_{0}$, it perceives a discrete increase in demand elasticity to $b+\alpha_{t-1}\left(p_{0}\right) \delta$, which lowers the optimal markup the firm targets. As long as costs do not increase too much, so that $c_{t} \leq \bar{c}_{t-1,0}$, the implied markup at $p_{0}$ is in fact still higher than the new target markup. Hence, the firm finds it optimal to keep its price fixed and let the markup decline. If the cost eventually moves higher than the threshold $\bar{c}_{t-1,0}$, the fall in markup would be too big, inducing the firm to change its price.

The logic is similar for a decrease in cost below $c_{0}^{*}$. As the firm entertains lowering its price from $p_{0}$, it perceives the discretely-flatter elasticity $b-\alpha_{t-1}\left(p_{0}\right) \delta$. Facing this decrease in elasticity, the firm finds it optimal to keep its price fixed and let the markup increase until $c_{t}$ falls to the lower bound $\underline{c}_{t-1,0}$. Only for a cost realization below this threshold is the implied increase in markup big enough to incentivize the firm to lower its price and move along the flatter demand curve it perceives below $p_{0}$.

Proposition 1 implies that rigidity arises if and only if there is ambiguity about the demand shape. If that is not the case, i.e. $\delta=0$, the interval of costs for which $p_{0}$ is the local optimizer is the singleton set $\left\{c_{0}^{*}\right\}$, and thus the probability that $p_{0}$ is a local maximizer becomes zero. With ambiguity, this probability becomes strictly positive. 
Unlike a fixed cost of changing the price, the as-if first-order perceived cost that emerges in our model is history dependent. There are two fundamental dimensions along which past information matters for this perception, which we now turn our attention to.

\section{Result \#2: The inaction region widens as a price gets observed more often}

The first dimension is that the perceived demand loss of changing the price increases with the signal-to-noise ratio $\alpha_{t-1}\left(p_{0}\right)$ (see Proposition 1). Intuitively, increasing the precision of the information available at $p_{0}$ makes the firm more confident in its estimate of $x\left(p_{0}\right)$, effectively amplifying the perceived increase in uncertainty when moving away from $p_{0}$. This translates in a larger difference between the worst-case demand elasticities on either side of $p_{0}$, which in turn raises the first-order loss of changing prices. Since $\alpha_{t-1}\left(p_{0}\right)$ increases with $N_{0}$, (by equation (7)), it follows that holding everything else constant, having seen the price $p_{0}$ more often in the past leads to a larger inaction region, as summarized in Corollary 2.

Corollary 2. The interval, defined in Corollary 1, of cost shock realizations $c_{t}$ for which $p_{0}$ is a local maximizer widens with $N_{0}$ :

$$
\frac{\partial \underline{c}_{t-1,0}}{\partial N_{0}}<0 ; \quad \frac{\partial \bar{c}_{t-1,0}}{\partial N_{0}}>0
$$

Proof. Follows from Corollary 1 and from $\frac{\partial \alpha_{t-1}\left(p_{t}\right)}{\partial N_{0}}>0$ in equation (7).

\section{Result \#3: Prices display memory}

Another crucial property of history dependence is that when past information $\varepsilon^{t-1}$ contains more than one unique price point, the general updating formulas discussed in Section 2.2 imply that there exist kinks in the as-if expected demand around each previously observed price level $p_{i} \in \varepsilon^{t-1}$. These kinks lead to qualitatively similar first-order losses in the expected profit around all such prices. This result is formalized in Proposition 2.

Proposition 2. Let $\delta_{i}^{*} \equiv \delta \operatorname{sgn}\left(p_{t}-p_{i}\right)$ for all $p_{i} \in \varepsilon^{t-1}$. For a given realization of $c_{t}$, up to a first-order approximation around each such $p_{i} \in \varepsilon^{t-1}$ :

$$
\ln v^{*}\left(\varepsilon^{t-1}, c_{t}, p_{t}\right)-\ln v_{0}^{*}\left(\varepsilon^{t-1}, c_{t}, p_{i}\right) \approx\left[\frac{e^{p_{i}}}{e^{p_{i}}-e^{c_{t}}}-\left(b+\alpha_{t-1, i}\left(p_{i}\right) \delta^{*}+A_{i}\right)\right]\left(p_{t}-p_{i}\right) .
$$

Proof. The switch in $\delta^{*}$ follows directly from the worst-case expected demand detailed in Online Appendix A.1. There we also define the term $\alpha_{t-1, i}(p)$ which denotes the weight put on the past demand realization at $p_{i}$ in the conditional expectation, i.e. its effective signal- 
to-noise ratio when updating with multiple signals. Lastly, $A_{i}$ collects additional derivative terms that do not depend on the sign of $\left(p_{t}-p_{i}\right)$, for details see Online Appendix A.2.

Letting $c_{i}^{*}=p_{i}-\ln \left(\frac{b}{b-1}\right)$ for all $p_{i} \in \varepsilon^{t-1}$, a direct counterpart to Corollary 1 follows.

Corollary 3. Under the first-order approximation in Proposition 2, for each $p_{i} \in \varepsilon^{t-1}$ there exists the interval $\left(\underline{c}_{t-1, i}, \bar{c}_{t-1, i}\right)$, where $\underline{c}_{t-1, i}=c_{i}^{*}+\ln \left[\frac{b}{b-1} \frac{b-\alpha_{t-1, i}\left(p_{i}\right) \delta+\hat{\alpha}-1}{b-\alpha_{t-1, i}\left(p_{i}\right) \delta+\hat{\alpha}}\right]$ and $\bar{c}_{t-1, i}=$ $c_{i}^{*}+\ln \left[\frac{b}{b-1} \frac{b+\alpha_{t-1, i}\left(p_{i}\right) \delta+\hat{\alpha}-1}{b+\alpha_{t-1, i}\left(p_{i}\right) \delta+\hat{\alpha}}\right]$, such that for all $c_{t} \in\left(\underline{c}_{t-1, i}, \bar{c}_{t-1, i}\right) p_{i}$ is a local maximizer.

Proof. For any $c_{t}$ in this interval, the first order derivative of the change in profits in Proposition 2 is negative for $p_{t}>p_{i}$ and positive for $p_{t}<p_{i}$, for all $p_{i} \in \varepsilon^{t-1}$.

Thus, Proposition 2 and Corollary 3 imply that the firm is not only reluctant to change its current price, but is in general inclined to repeat prices posted in the past, since there are kinks in the profit function there as well. This generates 'memory' in the price series.

\section{Result \#4: Good demand signals make a price change less likely}

Lastly, the analysis so far has focused on the first-order effect of price deviations around any of the $p_{i} \in \varepsilon^{t-1}$, showing that keeping the price fixed at previous levels is a local optimum. Next, we show that there is an additional interaction between the level of the past quantity sold $\bar{y}_{i}$ and the change in the perceived demand slope around the price $p_{i}$ at which that signal was observed. This interaction is of second-order, thus washes away in the local analysis above, but can matter for finding the global optimum, as we do in Section 4 .

This interaction arises from the fact that the signal-to-noise ratio $\alpha_{t-1, i}(p)$ declines with the distance between $p$ and $p_{i}$. Intuitively, because the levels of demand at different prices are imperfectly correlated, the information about demand at some price $p_{i}$ is most useful for updating beliefs at prices in its neighborhood. This naturally arises from the fact that demand does not come from a particular parametric family - when learning nonparametrically, information is inherently local, as it does not update beliefs about parameters that control the underlying function globally. The non-linearity of $\alpha_{t-1, i}(p)$ is of second-order locally, but matters when thinking about the global maximum.

The second cross-derivative of the worst-case expected demand, with respect to price and the perceived innovation at $p_{i}$, denoted by $\widehat{z}_{i} \equiv \bar{y}_{i}-\left(-\gamma-b p_{i}\right)$, is given by

$$
\frac{\partial^{2} \widehat{x}_{t-1}^{*}\left(p_{t} ; m^{*}\left(p ; p_{t}\right)\right)}{\partial p_{t} \partial \widehat{z}_{i}}=-2 \psi \alpha_{t-1, i}\left(p_{t}\right)\left(p_{t}-p_{i}\right) .
$$

Note that the derivative of the worst-case expected demand to the right (left) of $p_{i}$ becomes more negative (positive) as the perceived innovation $\widehat{z}_{i}$ increases. Hence, a higher 
signal innovation $\widehat{z}_{i}$ amplifies the effects of the endogenous switch in the worst-case demand elasticity. Intuitively, positive demand news shift up the conditional belief about demand at all prices, but the weight put on the signal decreases with $\left|p_{t}-p_{i}\right|$, so that beliefs about demand shift up the most locally. In Section 4 we investigate this interaction empirically and quantify how much a firm that observes a particularly good (bad) demand realization is more likely to keep (change) its posted price. This asymmetry in the effect of demand news on the probability of changing a price stands in contrast to most state-dependent mechanisms, such as a standard menu-cost model, where both positive and negative shocks make the firm more likely to reprice as they raise the gap between the current and optimal prices.

\subsection{Incorporating forward-looking behavior}

Next, we consider how forward-looking behavior affects optimal pricing, and stickiness in particular. The current price choice $p_{t}$ and demand realization $y_{t}$ become state variables in next period's problem, as they are incorporated in the future information set $\varepsilon^{t}$. This gives rise to a new incentive: posting a price for the sake of obtaining new information. ${ }^{10}$

To characterize this exploration motive, we need to analyze the continuation value in (5). This presents a technical problem - the relevant state $\varepsilon^{t-1}$ is the whole history of prices and demand realizations, which is infinitely long, thus making the general form of the dynamic problem intractable. To get around this, we assume the firm understands that its action today (time $t$ ) will change its information set in the future, but thinks that none of its future pricing decisions $(t+k)$ will affect its information set again - that is, $\varepsilon^{t+k}=\varepsilon^{t}, \forall k \geq 1$. We denote the resulting continuation value of the recursive problem from $t+1$ onward, when the firm does not face any more changes in the endogenous state $\varepsilon^{t}$ but still faces the fluctuations in exogenous cost process $c_{t+k}$, as $\tilde{V} \cdot{ }^{11}$ Plugging it into (5), the firm solves

$$
V\left(\varepsilon^{t-1}, c_{t}\right)=\max _{p_{t}} \min _{m(p) \in \Upsilon_{0}} E\left[\nu\left(\varepsilon_{t}, c_{t}\right)+\beta \int \tilde{V}\left(\varepsilon^{t}, c_{t+1}\right) g\left(c_{t+1} \mid c_{t}\right) d c_{t+1} \mid \varepsilon^{t-1}\right]
$$

This approximation makes the dynamic problem tractable, while featuring two important conceptual advantages. First, the firm is forward-looking into the discounted infinite future

\footnotetext{
${ }^{10}$ Conceptually, our environment is related to the multi-arm bandit literature. Here the payoffs of the arms (i.e. price choices) are correlated since $\psi>0$, and evaluated under multiple priors. See Bergemann and Valimaki (2008) for a survey of related applications of bandit problems studied under expected utility.

${ }^{11} \tilde{V}($.$) is the solution to the following recursive problem, with details presented in Online Appendix A.3$

$$
\tilde{V}\left(\varepsilon^{t}, c_{t+1}\right)=\max _{p_{t+1}} \min _{m(p) \in \Upsilon_{0}} E\left[\nu\left(\varepsilon_{t+1}, c_{t+1}\right)+\beta \int \tilde{V}\left(\varepsilon^{t}, c_{t+2}\right) g\left(c_{t+2} \mid c_{t+1}\right) d c_{t+2} \mid \varepsilon^{t}\right]
$$
}


in terms of the cost process $c_{t+k}$, hence does not only consider the likely cost next period as it would in a simple two-period model. Second, the approximation leaves the history $\varepsilon^{t-1}$ completely unrestricted. Thus, it avoids any ad hoc assumptions limiting the firms' memory, which could lead to built-in conclusions on how firms learn and the resulting pricing decisions. Instead, leaving it unrestricted allows us to evaluate in Section 4 the long-run properties of the model at its stochastic steady state, where that history is fully endogenous and long. In this section, however, we will focus on analyzing the qualitative features of the economic forces shaping the exploration motive, and to this end we treat the history $\varepsilon^{t-1}$ as given.

The experimentation motive is driven by a desire for information that is both new and relevant. On the one hand, the firm would like to obtain information on new parts of the demand curve where its ex-ante uncertainty is high. On the other hand, the firm values relevant information, i.e. signals that would affect beliefs about demand near prices that are likely to be posted in the future. The balance of these two forces determines whether the exploration incentives lead to the selection of a brand new price $p_{t}$ or revisiting one of the previously observed prices. Which one dominates depends crucially on the information the firm enters the period with $-\varepsilon^{t-1}$.

To gain analytical insight into this trade-off, we consider the special case where i) $\psi=\infty$, so that beliefs about demand at different prices are uncorrelated; and ii) there is perfect foresight that future costs are constant at some arbitrary level $c>0$, i.e. $c_{t+k}=c$ for all $k \geq$ 1. Under these assumptions, we can characterize analytically the expected continuation value $E\left[\tilde{V}\left(\left\{\varepsilon^{t-1}, p_{t}, y_{t}\right\}, c\right) \mid \varepsilon^{t-1}, p_{t}\right]$ as a function of $p_{t}$ (the expectation is over the realizations of the new signal $y_{t}$ ), and prove two results that illustrate how the exploration incentive could be maximized either away from or exactly at one of the previously observed prices.

The composition of the history of observations $\varepsilon^{t-1}$ is key to determining whether the optimal exploration strategy is to stay put or try something new. To illustrate, we consider two cases that would also help understand the numerical results in Section 4 where $\varepsilon^{t-1}$ is endogenous. First, let $\varepsilon^{t-1}=\varepsilon^{0}$ contain demand realizations at only one distinct price level $p_{0}$. To make the point starker, we assume that the realization of the observed signal $\bar{y}_{0}$ is good enough (i.e. $\left.\bar{y}_{0}>-\gamma-b p_{0}+\frac{\sigma_{x}^{2}}{2}\right)$, so that when the cost equals $c_{0}^{*}=p_{0}-\ln \left(\frac{b}{b-1}\right), p_{0}$ is not just locally optimal, but is in fact the global static profit-maximizer conditional on $\varepsilon^{0}$.

In Proposition 3, we characterize the current price $p_{t}$ that maximizes the expected continuation value when $c=\bar{c}_{0}$.

Proposition 3. The expected continuation value $E\left[\tilde{V}\left(\left\{\varepsilon^{0}, p_{t}, y_{t}\right\}, c_{0}^{*}\right) \mid \varepsilon^{0}, p_{t}\right]$ achieves its maximum at

$$
p_{t}^{*}=\arg \min _{p}\left(p-p_{0}\right)^{2} \text { s.t. } p \neq p_{0}
$$


Proof. We provide intuition in the text below, see Online Appendix A.3 for details.

Intuitively, choosing $p_{t}^{*}$ today ensures that the new signal $y_{t}$ will be informative about a price as close as possible to the ex-ante expected optimal $p_{0}$ - this makes the new information highly relevant. As a result, if the realization $\widehat{z}_{t}$ at the new signal is above a threshold $\bar{z}_{t}\left(p_{t}^{*}\right)$, characterized in the proof, then the firm will stick with this price in the future, set $p_{t+k}=p_{t}^{*}$, and take advantage of the unexpectedly high demand at that price while remaining near its ex-ante optimal markup level. On the other hand, if the signal realization happens to be bad, the firm can safely switch back to the ex-ante optimal $p_{0}$, where the belief about demand is not affected by $\widehat{z}_{t}$, and still offers lower uncertainty and the preferred markup.

The reason for not picking $p_{t}=p_{0}$ is that a bad realization of the new signal erodes the ex-ante best pricing option, $p_{0}$, while the firm does not have a good fall-back alternative, as it has no observations of demand at other prices. Because of this, it is best to experiment with a brand new price, though the desire for relevant information keeps the firm near $p_{0}$.

Proposition 3 describes a case where the value of new information is maximized away from $p_{0}$. However, next we show that this is not a general result, but depends on whether the firm has seen one or more distinct prices in the past. In particular, let $\varepsilon^{t-1}=\varepsilon^{1}$ contain demand realizations at two distinct prior prices, $p_{0}$ and $p_{1}$. Also, to simplify the exposition we assume that the information received at these prices is of the same quality - demand at each price has been observed the same number of times $\left(N_{1}=N_{0}\right)$, and the observed signals, $\bar{y}_{0}$ and $\bar{y}_{1}$, imply equally-good news, i.e. the same perceived innovation: $\widehat{z}_{0}=\widehat{z}_{1}=\widehat{z}$.

Proposition 4 shows that when the previously observed demand at $p_{0}$ and $p_{1}$ has been good enough, the continuation value is maximized at $p_{0}$ for a range of cost shocks around $c_{0}^{*}$. Thus, forward-looking behavior reinforces the static stickiness result (Corollary 1).

Proposition 4. There is a non-singleton interval of costs $(\underline{c}, \bar{c})$ around $c_{0}^{*}$, and a threshold $\chi>0$, such that if $\widehat{z}>\chi$, then for any $c \in(\underline{c}, \bar{c})$ :

$$
p_{0}=\arg \max _{p_{t}} E\left[\tilde{V}\left(\left\{\varepsilon^{1}, p_{t}, y_{t}\right\}, c\right) \mid \varepsilon^{1}\right] .
$$

Moreover, the threshold $\chi$ is decreasing in $\left|p_{1}-p_{0}\right|$.

Proof. We provide intuition in the text below, see Online Appendix A.3 for details.

The reason for this result is two-fold. First, information about demand at $p_{0}$ is the most relevant since that is the price expected to be optimal in the future. Second, even if the firm receives a 'disappointing' new signal $y_{t}$ at $p_{0}$, it has a good fall-back option as it has also accumulated information (and thus reduced uncertainty) at the price level $p_{1}$. Thus, the firm can set $p_{t}=p_{0}$ and further reduce uncertainty about demand at the most likely future price, 
safe with the knowledge that it has a good alternative in case the new information is bad. The value of the fall-back option is important - in particular, the perceived innovation in the average past demand realization at $p_{1}$ must exceed a threshold $\chi$ (which we characterize in the proof). This threshold is lower when $p_{0}$ and $p_{1}$ are closer to each other, because then their implied markups are more similar, making the two price choices closer substitutes, and thus $p_{1}$ a more attractive fall-back option.

Our analytical results show that forward-looking behavior can both counteract and reinforce the previous stickiness result derived from static maximization. The resulting

overall effect depends crucially on the structure of the prior history $\varepsilon^{t-1}$, which highlights the importance of taking into account the endogeneity of that history. To that end, Section 4 numerically analyzes the stochastic steady state of a general version of our forward-looking model, with $\psi<\infty$ and stochastic cost shocks. We find that experimentation is not only consistent with significant price stickiness, but also helps generate an empirically relevant (i) life-cycle profile of pricing behavior and (ii) size distribution of price changes.

\section{Quantitative Model and Nominal Rigidity}

In this section, we embed our mechanism in a macroeconomic model with monopolistic competition. The key elements are that firms are uncertain about both (i) their demand curve and (ii) the competitors' price index. We first show analytically that this two-dimensional uncertainty gives rise to as-if kinks in demand in terms of nominal prices. Then, in the next section, we quantify the ability of our mechanism to match micro-level moments and generate monetary non-neutrality. In what follows, all lower case variables are in logs.

\subsection{Structure of competition}

The first primitive of the economic framework is the firm's set of direct competitors. We assume that firm $i$ sells to a single industry $j$ and in doing so, competes against a continuum of other monopolistically competitive firms who do the same. Each industry $j$ has a representative final-good firm that aggregates the varieties $i$. Its cost-minimization problem implies a demand schedule $x_{j}($.) for the good of firm $i$ in industry $j$

$$
y_{i, t}=x_{j}\left(p_{i, t}-p_{j, t}, y_{j, t}, z_{i, t}\right)
$$


where $p_{i, t}$ is the $\log$ price set by firm $i$, and the log industry price index $p_{j, t}$ is such that $e^{p_{j, t}+y_{j, t}}=\int e^{p_{i, t}+y_{i, t}} d i{ }^{12}$ The $z_{i, t}$ term is an idiosyncratic demand shock for good $i$ which is unobserved by firm $i$ but known to be distributed as $N\left(0, \sigma_{z}^{2}\right)$. The demand curve in equation (11) is a generalization of the typical CES structure, with the familiar result that the demand for a given intermediate good $i$ is a function of the firm's price relative to the industry average, $p_{i, t}-p_{j, t}$; overall industry output $y_{j, t}$; and demand shocks $z_{i, t}$.

At the aggregate level, a representative household consumes a final good produced by a competitive firm that buys from the continuum of industries $j$. The household's consumption basket and the associated aggregate price index are given by the standard CES structures $y_{t}=\frac{b}{b-1} \ln \left(\int e^{y_{j, t} \frac{b-1}{b}} d j\right)$ and $p_{t}=\frac{1}{1-b} \ln \left(\int e^{p_{j, t}(1-b)} d j\right)$. Cost minimization by the final good producer implies a standard demand curve for the industry $j$ composite good

$$
y_{j, t}=y_{t}+b\left(p_{t}-p_{j, t}\right)
$$

We denote the relative prices that enter as arguments in the demand curves for firm $i$ in equation (11) and for industry $j$ in equation (12), respectively, as

$$
r_{i, t} \equiv p_{i, t}-p_{j, t} ; \quad r_{j, t} \equiv p_{t}-p_{j, t}
$$

\subsection{Information about competition}

We model a firm that has Knightian uncertainty over the joint assessment of (i) its demand curve $x_{j}$ as a function of its own relative price $p_{i, t}-p_{j, t}$, and (ii) the price index of its direct competitors $p_{j, t}$. Each firm $i$ observes the full history of its own prices and quantities, $p_{i, t}$ and $y_{i, t}$, as well as the aggregate output and price levels, $y_{t}$ and $p_{t}$. Intuitively, our framework is meant to capture the idea that since firms do not know the exact structure of the demand they face, they also do not know how to precisely aggregate over the prices of their direct competitors to build the relevant price index they compete against. Thus, uncertainty about the competitive environment manifests itself in uncertainty over the shape of the demand curve, but also the relevant price index that determines a specific firm's relative price.

\footnotetext{
${ }^{12}$ In the background, the technology is modeled as $e^{y_{j, t}}=f_{j}^{-1}\left(\int f_{j}\left(e^{y_{i, t}}\right) g_{j}\left(e^{z_{i, t}}\right) d i\right)$, where each industry $j$ has potentially different production functions $f_{j}$ and $g_{j}$. Solving the cost-minimization problem of the final good firm in industry $j$ yields $y_{i, t}=\ln \left[f_{j}^{\prime-1}\left(e^{p_{i, t}-p_{j, t}} \frac{f_{j}^{\prime}\left(e^{y_{j, t}}\right)}{g_{j}\left(e^{z i, t}\right)}\right)\right]$. In equation (11) we summarize the effective demand curve as $x_{j}$ and note that it is a transformation of the functions $f_{j}$ and $g_{j}$.
} 


\section{Ambiguity about the demand curve}

For tractability, we assume the firm understands that the industry demand $y_{j, t}$ and the demand shocks $z_{i, t}$ enter multiplicatively in the unknown function $x_{j}$ in equation (11). Since firm $i$ also knows the structure of the aggregate consumption basket, it can substitute out industry output $y_{j, t}$ from equation (12) to obtain the demand schedule

$$
y_{i, t}=x_{j}\left(r_{i, t}\right)+b r_{j, t}+y_{t}+z_{i, t}
$$

where the relative prices $r_{i, t}$ and $r_{j, t}$ are defined in (13).

Ambiguity about the demand curve $x_{j}$ is modeled as in equations (3) and (4): there is a set of multiple priors, each of which is a GP distribution with mean function $m\left(r_{i}\right)$ so that

$$
m\left(r_{i}\right) \in\left[-\gamma-b r_{i}, \gamma-b r_{i}\right] ; \quad m^{\prime}\left(r_{i}\right) \in[-b-\delta,-b+\delta]
$$

\section{Ambiguity about the relative price}

In our model, the firm does not directly observe its direct competitors' price index $p_{j, t}$. It does, however, have two relevant sources of information. These sources differ in the perceived ambiguity about their informational content. In particular, the firm is confident, i.e perceives no ambiguity, about the first source, which consists of marketing reviews that perfectly reveal the value of $p_{j, t}$. We model reviews as occurring with some exogenous probability $\lambda_{T}$. Here we implicitly assume that there are some technological constraints on the ability to perform frequent reviews (e.g. the necessary data may not be observed every period); or simply that reviews are costly, leading the firm to perform them infrequently. ${ }^{13}$

In addition, the firm observes the aggregate price level $p_{t}$ at all time. However, unlike in a rational expectations ( $\mathrm{RE}$ ) framework, we assume the firm is not confident about how $p_{t}$ relates to the unknown $p_{j, t}$, and perceives their relationship as ambiguous. In particular, we assume that while the firm is certain that aggregate and industry prices are cointegrated and thus must keep pace with each other in the long-run, the firm is uncertain in the shortrun structural relationship between the two. Putting together the firm's two sources of information, it perceives the evolution of $p_{j t}$ as

$$
p_{j, t}=\widetilde{p}_{j, t}+\phi\left(p_{t}-\widetilde{p}_{j, t}\right)
$$

\footnotetext{
${ }^{13}$ As long as reviews do not happen every period, using deterministic or state-dependent review lags would not change our analysis significantly. The modeling advantage over a deterministic timing is computational: we find that stochastic review times achieve faster convergence towards the stationary distribution. The advantage over a state-dependent setup is tractability, as it avoids modeling a cost-benefit analysis of reviews.
} 
where $\widetilde{p}_{j, t}$ is the most recent review signal as of time $t$, and the function $\phi$ summarizes the unknown and ambiguous structural relationship between $p_{t}$ and $p_{j, t}$. Indeed, our assumption that firms do not know the exact industrial structure (i.e. the function $x_{j}$ ) implies that they also do not know the exact equilibrium relationship between $p_{t}$ and $p_{j, t}$ - different industry production functions imply different such structural relationships.

Ambiguity about $\phi$ is modeled with the same tools as the uncertainty about the demand function $x_{j}$. Specifically, we assume that the priors on $\phi$ are GP distributions, with mean functions that lie in a set $\Omega_{\phi}$ around the true DGP $\phi\left(p_{t}-\widetilde{p}_{j, t}\right)=p_{t}-\widetilde{p}_{j, t}$. For tractability, we focus on the limiting case in which the variance function of the GP distributions over $\phi$ goes to zero almost everywhere. Given the resulting Dirac priors, we can simplify notation and specify the set of priors directly as a set of possible $\phi$ 's the firm entertains.

In particular, we specify that for small inflationary pressure, i.e. when $\left|p_{t}-\widetilde{p}_{j, t}\right|$ is less than some threshold $\Gamma$, the relationship is uncertain and the function $\phi$ lies in the interval

$$
\phi\left(p_{t}-\widetilde{p}_{j, t}\right) \in\left[-\gamma_{p}, \gamma_{p}\right], \text { for }\left|p_{t}-\widetilde{p}_{j, t}\right| \leq \Gamma
$$

This captures the idea that observing a small change in the aggregate price $p_{t}$ does not convince the firm that the unobserved industry price has also changed. Online Appendix A.7, shows that such uncertainty about the local relationship between aggregate and industry inflation is well supported by the data. Lastly, to ensure that under all admissible priors $p_{t}-p_{j, t}$ is stationary, we make the set of potential $\phi$ grow with $p_{t}-p_{j, t}$ as inflation rises:

$\phi\left(p_{t}-\tilde{p}_{j, t}\right) \in\left[-\gamma_{p}+p_{t}-\tilde{p}_{j, t}-\Gamma \operatorname{sgn}\left(p_{t}-\tilde{p}_{j, t}\right), \gamma_{p}+p_{t}-\tilde{p}_{j, t}-\Gamma \operatorname{sgn}\left(p_{t}-\tilde{p}_{j, t}\right)\right]$, for $\left|p_{t}-\tilde{p}_{j, t}\right| \geq \Gamma$.

\section{Unambiguous estimates of relevant relative prices and demand}

The review signal $\widetilde{p}_{j, t}$ is the only unambiguous estimate of $p_{j, t}$. The firm can use this signal to construct unambiguous estimates of the relative prices of interest, $r_{i, t}$ and $r_{j, t}$, as

$$
\widetilde{r}_{i, t} \equiv p_{i, t}-\widetilde{p}_{j, t} ; \quad \widetilde{r}_{j, t} \equiv p_{t}-\widetilde{p}_{j, t}
$$

Here, $\widetilde{r}_{i, t}$ represents the firm's estimate of the relevant relative price driving its own demand curve, constructed using the firm's observed nominal price $p_{i, t}$ and the review signal $\widetilde{p}_{j, t}$. In turn, $\widetilde{r}_{j, t}$ is the estimate of the relative price that enters the industry $j$ demand curve.

Using these expressions, we can decompose the relative prices $r_{i, t}$ and $r_{j, t}$ into a component over which the firm is confident and one that is perceived as ambiguous. Specifically, given the law of motion of $p_{j, t}$ in equation (16), the unknown relative prices $r_{i, t}$ and $r_{j, t}$ 
defined in (13), and their unambiguous estimates in (18), the decomposition is given by

$$
r_{i, t}=\widetilde{r}_{i, t}-\phi\left(\widetilde{r}_{j, t}\right) ; \quad r_{j, t}=\widetilde{r}_{j, t}-\phi\left(\widetilde{r}_{j, t}\right)
$$

Substituting the decompositions of the unobserved $r_{i, t}$ and $r_{j, t}$ in (19) into the demand equation (14) leads to

$$
y_{i, t}=\underbrace{x_{j}\left(\widetilde{r}_{i, t}-\phi\left(\widetilde{r}_{j, t}\right)\right)-b \phi\left(\widetilde{r}_{j, t}\right)}_{\text {ambiguous components of demand }}+b \widetilde{r}_{j, t}+y_{t}+z_{i, t},
$$

which isolates the unambiguous and ambiguous components of demand. The former are given by the observed aggregate output $y_{t}$, the risky demand shock $z_{i, t}$ and the unambiguous estimates of the relative prices $\left(\widetilde{r}_{i, t}, \widetilde{r}_{j, t}\right)$. The ambiguous components are due to ambiguity over (i) the demand curve $x_{j}$ itself, with the set of priors given in equation (15); and over (ii) the relative prices $\left(r_{i, t}, r_{j, t}\right)$, arising from the set of priors for $\phi$ in (17).

\subsection{Optimization problem}

Having described the competitive and information structures, we now turn our attention to the profit maximization problem. We assume that the production function of the intermediate-good firm $i$ is given by $y_{i, t}=\omega_{i, t}+a_{t}+l_{i, t}$, where $\omega_{i, t}$ and $a_{t}$ are an idiosyncratic and aggregate productivity shock respectively; and $l_{i, t}$ represents the (log) hours supplied by the household to firm $i$. The processes for these shocks are known:

$$
\omega_{i, t}=\rho_{\omega} \omega_{i, t-1}+\varepsilon_{i, t}^{\omega} ; \quad a_{t}=\rho_{a} a_{t-1}+\varepsilon_{t}^{a}
$$

where $\varepsilon_{i, t}^{\omega}$ is iid $N\left(0, \sigma_{\omega}^{2}\right)$ and $\varepsilon_{t}^{a}$ is iid $N\left(0, \sigma_{a}^{2}\right)$. Because we are ultimately interested in how nominal shocks affect the firm's pricing decisions, nominal spending is the other aggregate shock in this economy: the log nominal aggregate spending $s_{t}=p_{t}+y_{t}$ is assumed to follow a random walk with drift, $s_{t}=\mu+s_{t-1}+\epsilon_{t}^{s}$, where $\epsilon_{t}^{s}$ is iid $N\left(0, \sigma_{s}^{2}\right)$.

The aggregate side of the model is standard. We assume linear labor disutility for the representative household, which then equalizes the wage to consumption, which in turn is simply equal to aggregate output by market clearing (as detailed in the Online Appendix A.4). Given the log-demand in equation (20), the real flow profit of firm $i$ becomes

$$
v_{i, t}=\left(e^{p_{i, t}-p_{t}}-e^{y_{t}-\omega_{i, t}}\right) e^{y_{i, t}} .
$$

The firm's problem can be summarized as follows: The firm enters period $t$ with 
knowledge of the history of all its previous quantities sold, the corresponding nominal prices at which those quantities were observed and its history of industry price review signals. In addition, the firm sees the history of aggregate prices and output. At the start of time $t$, the idiosyncratic productivity $\omega_{i, t}$ and aggregates $\left(p_{t}, y_{t}\right)$ are observed. If a new review is conducted, $\widetilde{p}_{j, t}$ equals $p_{j, t}$ and equals $\widetilde{p}_{j, t-1}$ otherwise. Given the history of observables and current states, the firm optimizes over its action, $p_{i, t}$, taking into account the ambiguity over its demand in equation (20). At the end of period $t$, the demand shock $z_{i, t}$ is realized and the firm updates its information set with the observed quantity sold $y_{i, t}$ at price $p_{i, t}$.

Finally, the firm needs to conjecture a law of motion of $p_{t}$ to forecast future profits. We close the model by assuming that the ambiguity-averse firms are measure zero, while the rest of the economy is populated by rational-expectations firms. This makes aggregate determination simple, as the equilibrium $p_{t}$ is given by $p_{t}^{R E}$. The implication of this assumption is that by ignoring strategic complementaries in price setting, the quantitative benchmark of Section 4 can be seen as a lower bound on the degree of monetary non-neutrality.

For a transparent comparison between our model and the RE benchmark, we assume a simple DGP where each variety $i$ faces the same demand function coming from industry $j$ in (14), given by $x_{j}\left(r_{i, t}\right)=-b r_{i, t}$. Using this knowledge of the demand function in equation (20), it follows that a RE firm has full knowledge that its demand is $y_{i, t}^{R E}=-b\left(p_{i, t}-p_{t}\right)+y_{t}+z_{i, t}$. The resulting optimal $\mathrm{RE}$ nominal price takes the familiar form $p_{i, t}^{R E}=\log \frac{b}{b-1}+p_{t}-\omega_{i, t}$, where the aggregate price (up to a constant) is $p_{t}^{R E}=s_{t}-a_{t}$.

\section{Aggregate price level and profits}

Note that the aggregate price $p_{t}$ affects real profits in equation (21) through three possible channels. The first is the standard effect of deflating nominal profits by $p_{t}$. The other two effects show up in the demand equation (20). On the one hand, when observing a higher $p_{t}$, holding $\widetilde{p}_{j, t}$ constant, the firm estimates that the industry $j$ 's composite good is relatively cheaper. As a result, the firm expects higher demand for industry $j$ 's composite good, which in turn, holding everything else constant, translates into a higher demand for firm $i$. This demand shifter is given by $b \widetilde{r}_{j, t}$ in equation (20). On the other hand, the same observation of a higher $p_{t}$ may change the firm's perception of the unobserved $p_{j, t}$, through their structural relationship $\phi\left(\widetilde{r}_{j, t}\right)$. In particular, holding constant $\widetilde{p}_{j, t}$, a larger $p_{t}$ indicates to the firm that the aggregate price index is higher than the firm's unambiguous estimate of $p_{j, t}$. While in a

RE model this observation would fully convince the firm that $p_{j, t}$ must have also risen, our ambiguity-averse firm lacks such confidence, as summarized in the set of beliefs in (17). 


\subsection{Joint worst-case beliefs}

To illustrate the key intuition analytically, for the rest of this section we zero-in on the special case of a myopic firm born at time $t=0$ that is in its second period of life (i.e. $t=1$ ). Hence, its information set contains just one previous price point, $p_{i, 0}$, and the quantity sold at that price, $y_{i, 0} \cdot{ }^{14}$ In addition, the firm observes the history of aggregates, $\left\{y_{0}, y_{1}, p_{0}, p_{1}\right\}$, and signals on the industry price level, $\left\{\widetilde{p}_{j, 0}, \widetilde{p}_{j, 1}\right\}$. These observables are used by the firm to form the unambiguous estimates of the relative prices, $\left\{\widetilde{r}_{i, 0}, \widetilde{r}_{j, 0}, \widetilde{r}_{j, 1}\right\}$, as defined in (18).

Abstracting from the unambiguous terms, expected demand is given by

$$
m\left(\widetilde{r}_{i, 1}-\phi\left(\widetilde{r}_{j, 1}\right)\right)-b \phi\left(\widetilde{r}_{j, 1}\right)+\alpha\left\{y_{i, 0}-\left[m\left(\widetilde{r}_{i, 0}-\phi\left(\widetilde{r}_{j, 0}\right)\right)-b \phi\left(\widetilde{r}_{j, 0}\right)\right]\right\},
$$

where $\alpha=\frac{\sigma_{x}^{2}}{\sigma_{x}^{2}+\sigma_{z}^{2}} .15$ The expectation is simply a combination of a prior belief about demand at the current relative price $r_{i, 1}$ (i.e. $\left.m\left(\widetilde{r}_{i, 1}-\phi\left(\widetilde{r}_{j, 1}\right)\right)-b \phi\left(\widetilde{r}_{j, 1}\right)\right)$ and an update given the realized quantity $y_{i, 0}$ at the previous price $r_{i, 0}$. Since the industry prices $p_{j, 0}$ and $p_{j, 1}$ are uncertain, expected demand depends on the priors for both the demand curve, $m($.$) , and$ the structural relationship between industry and aggregate prices, $\phi($.$) .$

The analysis of the worst-case priors is similar to the one in the real model in that it can be decomposed in jointly considering the worst-case priors for (i) the level of demand at the current relative price $r_{i, 1}$ and (ii) the change in demand between $r_{i, 1}$, and the previous $r_{i, 0}$ (where the quantity sold $y_{i, 0}$ was observed). However, the firm now needs to account for the joint ambiguity over both the demand shape, i.e. $m$, and the industry-aggregate price relationship $\phi$, since the latter affects perceptions about the unknown $p_{j, 0}$ (the industry price when $y_{i, 0}$ was realized) and $p_{j, 1}$ (the industry price that determines the current relative price).

The worst-case prior for the level of demand at the current price $r_{i, 1}$ is straightforward it is equal to the lower bound of (15), given by $-\gamma-b\left(\widetilde{r}_{i, 1}-\phi\left(\widetilde{r}_{j, 1}\right)\right)$. Under this prior, the first two components of expected demand in (22) simplify to $-\gamma-b \widetilde{r}_{i, 1}$, as the $b \phi\left(\widetilde{r}_{j, 1}\right)$ terms cancel out. This reflects our assumption that while the firm faces ambiguity about the local elasticity of its demand schedule, its beliefs are centered around the true $x_{j}\left(r_{i, t}\right)=-b r_{i, t}$.

Still, the ambiguity about the shape of demand impacts the firm's evaluation of the change in demand from $r_{i, 1}$ to $r_{i, 0}$, and thus its update based on the realization of $y_{i, 0}$. In

\footnotetext{
${ }^{14}$ In Online Appendix B.2 (on the authors' website) we show how the conceptual analysis is extended to having multiple past prices, and Section 4 shows numerical results for the general case of forward-looking firms with unrestricted history.

${ }^{15}$ To simplify notation and the analysis, for the rest of this section we suppress the local information effects by working with $\psi=0$. We relax this assumption in Section 4 .
} 
fact, there are two sources of ambiguity affecting this update, since the surprise in $y_{i, 0}$ is

$$
y_{i, 0}-[\underbrace{m\left(\widetilde{r}_{i, 0}-\phi\left(\widetilde{r}_{j, 0}\right)\right)-m\left(\widetilde{r}_{i, 1}-\phi\left(\widetilde{r}_{j, 1}\right)\right)}_{\text {Ambiguous change along firm's demand curve }}]+\underbrace{b\left[\phi\left(\widetilde{r}_{j, 0}\right)-\phi\left(\widetilde{r}_{j, 1}\right)\right]}_{\text {Ambiguous change in industry's relative price }}
$$

The first source of ambiguity in the update is the firm's uncertainty over the shape of its demand curve between $r_{i, 0}$ and $r_{i, 1}$, governed by the set of priors $m$. The second source of ambiguity is due to the unobserved change in the industry price level from $p_{j, 0}$ to $p_{j, 1}$, and is governed by the set of priors on the industry-aggregate price structural relationship $\phi$. Moreover, the unknown industry inflation impacts the firm's update in two places: (i) it affects the actual change in the relative price $r_{i, t}$ and enters the argument of the prior on the demand function $m$, and (ii) introduces a shift in the firm's demand schedule, as movements in $p_{j, t}$ against the aggregate price $p_{t}$ change the economy's demand for the composite good of industry $j$, shifting demand for all firms in that industry (the second term in (23)).

Once the relevant constraints on the admissible functions $m$ and $\phi$ are taken into account, determining the joint worst case for the ambiguous update in (22) reduces to

$$
\min _{\delta^{\prime} \in[-\delta, \delta]} \min _{\phi\left(\widetilde{r}_{j, t}\right) \in\left[-\gamma_{p}, \gamma_{p}\right]}-\delta^{\prime}\left\{\widetilde{r}_{i, 1}-\widetilde{r}_{i, 0}-\left[\phi\left(\widetilde{r}_{j, 1}\right)-\phi\left(\widetilde{r}_{j, 0}\right)\right]\right\}
$$

The joint worst-case prior beliefs depend on whether the firm considers a price that raises or lowers $\widetilde{r}_{i, 1}$ relative to $\widetilde{r}_{i, 0}$. When it entertains an action that increases $\widetilde{r}_{i, t}$, it sets in motion a concern that its effective demand is sensitive to this action. This concern manifests itself in a joint worst-case belief that both (i) the unknown demand curve $m$, is steep, i.e. $\delta^{*}=\delta$, and that (ii) there was a decline in the unknown price index of its direct competition within industry $j$. Hence, if $\widetilde{r}_{i, 1} \geq \widetilde{r}_{i, 0}$, the minimizing priors are

$$
\delta^{*}=\delta ; \quad \phi^{*}\left(\widetilde{r}_{j, 1}\right)=-\gamma_{p} ; \quad \phi^{*}\left(\widetilde{r}_{j, 0}\right)=\gamma_{p}
$$

In contrast, when the firm entertains decreasing its estimated relative price $\widetilde{r}_{i, 1}$, it worries about the opposite situation: that (i) its unknown demand curve is flat (i.e. $\delta^{*}=-\delta$ ), and (ii) it is facing an increase in the unknown price index of the competition. Hence, if $\widetilde{r}_{i, 1} \leq \widetilde{r}_{i, 0}$,

$$
\delta^{*}=-\delta ; \quad \phi^{*}\left(\widetilde{r}_{j, 1}\right)=\gamma_{p} ; \quad \phi^{*}\left(\widetilde{r}_{j, 0}\right)=-\gamma_{p}
$$

It is worth pointing out that the worst-case belief about the change in $p_{j, t}$ is not always that the firm's competition has lowered prices. The reason is that the industry price affects the firm's demand in two ways: (i) it determines the relevant relative price (the argument of $x_{j}$ ), and (ii) acts as a demand shifter since lower overall prices in industry $j$ boost demand 
for all firms inside the industry. These two effects go in opposite directions, and which one dominates depends on the perceived elasticity of the demand function $x_{j}$. When $x_{j}$ takes on the average elasticity $b$, these two effects cancel out. However, when the firm is contemplating a price increase or decrease, the worst-case local elasticity of $x_{j}$ changes away from $b$. As a result, the worst-case belief about $\phi$ endogenously depends on the firm's action $\widetilde{r}_{i, 1}$.

The key implication of equations (25) and (26) is that the joint worst-case beliefs over the demand curve and the industry-aggregate price relationship endogenously induce a kink in the worst-case conditional demand schedule around $\widetilde{r}_{i, 0}$, - notice they give rise to an absolute value term $-\alpha \delta\left|\widetilde{r}_{i, 1}-\widetilde{r}_{i, 0}\right|$ in (24). The reason behind the emergence of the kink is similar to the real model, and in particular equation (10). The difference is that while in the real model the relevant relative price was uniquely determined by the firm's action, here this is not the case. Intuitively, the firm is facing an identification problem, as it is uncertain about both the argument and the shape of the demand function. Faced with this joint ambiguity, it turns out that the robust solution is to estimate the demand curve in terms of the unambiguous estimate of the relative price $\widetilde{r}_{i, t}$. Due to the uncertainty about the local shape of the demand function, a kink at the previously observed $\widetilde{r}_{i, 0}$ emerges.

\subsection{Learning and nominal rigidity}

The kink in the worst-case expected demand leads to a first-order expected loss of having an estimated relative price $\widetilde{r}_{i, 1}$ different from $\widetilde{r}_{i, 0}$. The firm can avoid this loss by posting a nominal price $p_{i, 1}$ such that $\widetilde{r}_{i, 1}=\widetilde{r}_{i, 0}$, or

$$
p_{i, 1}-\widetilde{p}_{j, 1}=p_{i, 0}-\widetilde{p}_{j, 0}
$$

Naturally, rigidity in $\widetilde{r}_{i, t}$ has implications for the optimal nominal price.

First, consider the case in which a new review of the industry price does not occur at time $t=1$, so that $\widetilde{p}_{j, 1}=\widetilde{p}_{j, 0}$. In that case, if the firm finds it optimal to take advantage of the kink in estimated relative prices, equation (27) shows that the firm will do so by keeping its nominal price fixed and set $p_{i, 1}=p_{i, 0}$. This makes the nominal price rigid.

Of particular importance is the result that the optimal nominal price $p_{i, 1}^{*}$ may stay fixed at its previous value $p_{i, 0}$ even as the aggregate price changes. The current aggregate price $p_{1}$ is one of the state variables that affects profits, as shown in equation (21) and discussed in Section 3.3, and thus affects pricing decisions. However, due to the kink in the worst-case expected demand, captured by the $-\alpha \delta\left|\widetilde{r}_{i, 1}-\widetilde{r}_{i, 0}\right|$ term discussed above, we show (see details in Proposition A1 in Online Appendix A.5) that there is a range of values of aggregate inflation for which it is optimal for the firm to keep $p_{i, 1}=p_{i, 0}$. A similar argument leads to 
nominal rigidity conditional on changes in the other state variables as well.

Alternatively, consider periods when the firm observes the industry price through a review signal, i.e. $\widetilde{p}_{j, 1}=p_{j, 1}$. To take advantage of the kink at $\widetilde{r}_{i, 0}$, the firm changes its nominal price away from $p_{i, 0}$ in response to the information that is revealed by the difference $p_{j, 1}-\widetilde{p}_{j, 0}$. Unless by chance $p_{j, 1}=\widetilde{p}_{j, 0}$, rigidity in the estimated relative price leads to a nominal adjustment.

\section{Indexation is suboptimal}

Our model makes indexation to the aggregate price level suboptimal, even in the absence of external costs of changing prices. Intuitively, this is because the unambiguous estimate of the relevant relative price, $p_{i, t}-\widetilde{p}_{j, t}$, is conceptually different from the real price, $p_{i, t}-p_{t}$. The firm optimally seeks to minimize exposure to ambiguity about its demand. Since demand is a function of the relative price $p_{i, t}-p_{j, t}$, the firm optimally keeps constant the unambiguous estimate of this relative price, not the real price as measured against the aggregate price. The two concepts of relative prices are materially different in our model because the observed aggregate price $p_{t}$ is neither (i) the direct competitors' price index, nor (ii) an unambiguous estimate of it.

To see this, note that the observed aggregate price can be used to deflate all nominal prices to obtain real prices. We denote the resulting real versions of the price of the firm, the current industry $j$ price, and the industry price observed at the last marketing review as

$$
p_{i, t}^{\text {real }} \equiv p_{i, t}-p_{t} ; \quad p_{j, t}^{\text {real }} \equiv p_{j, t}-p_{t} ; \quad \widetilde{p}_{j, t}^{\text {real }} \equiv \widetilde{p}_{j, t}-p_{t}
$$

The key point is that as long as the competitive and information structure defined in Sections 3.1 and 3.2 are maintained, such re-normalization of prices based on variables in the firm's information set leads to the same optimal solution as derived above. Therefore, the nominal rigidity is not an artifact of a lack of properly deflating nominal prices or nominal illusion.

We can rewrite all of the basic relationships of our model using the deflated prices in (28). For example, the demand curve faced by the individual firm can be written as

$$
y_{i, t}=x_{j}\left[p_{i, t}^{\text {real }}-\widetilde{p}_{j, t}^{\text {real }}-\phi\left(-\widetilde{p}_{j, t}^{\text {real }}\right)\right]-b\left[\widetilde{p}_{j, t}^{\text {real }}+\phi\left(-\widetilde{p}_{j, t}^{\text {real }}\right)\right]+y_{t}+z_{i, t}
$$

where the set of beliefs over the functions $x_{j}($.$) and \phi($.$) is the same as in (15) and (17).$ Indeed, in the short run the firm still entertains that $\phi\left(-\widetilde{p}_{j, t}^{\text {real }}\right) \in\left[-\gamma_{p}, \gamma_{p}\right]$. In our two-period case, equation (29) leads to a kink in the worst-case expected demand around the estimated 
relative real price $p_{i, 0}^{\text {real }}-\widetilde{p}_{j, 0}^{\text {real }}$. The local profit maximizer around that kink is to set

$$
p_{i, 1}^{\text {real }}-p_{i, 0}^{\text {real }}=\widetilde{p}_{j, 1}^{\text {real }}-\widetilde{p}_{j, 0}^{\text {real }}
$$

But since aggregate inflation $p_{1}-p_{0}$ cancels out on both sides of this equation, we obtain back the optimal nominal price solution given by equation (27).

Intuitively, when there is no review, the RHS of equation (30) implies that the observed aggregate inflation rate reduces the unambiguous estimate of the industry $j$ real price oneto-one. To keep demand at the same estimated real price relative to the industry average, the firm finds it optimal to keep its nominal price unchanged, i.e. $p_{i, 1}=p_{i, 0}$, and thus let its real price also decrease one-to-one, as implied by the LHS of equation (30).

Suppose on the contrary that the firm were to index its nominal price using aggregate inflation and follow a pricing policy that sets $p_{i, 1}^{\text {real }}=p_{i, 0}^{\text {real }}$, and keeps its real price constant. Positive aggregate inflation implies a lower estimate of the real industry price $\widetilde{p}_{j, 1}^{\text {real }}$, compared to the last period. Keeping its own real price constant while that estimate has decreased is equivalent to an increase in the firm's real relative price vis-a-vis its direct within industry competitors, i.e. $p_{i, 1}^{\text {real }}-\widetilde{p}_{j, 1}^{\text {real }}$ goes up. This is precisely what the firm wishes to avoid, as movements in this relative price expose it to first-order losses arising from the ambiguity about the demand shape, as discussed above. ${ }^{16}$ Thus, such indexation is suboptimal.

\section{Stickiness and memory in nominal prices}

The result of equation (27) extends in a straightforward fashion once we move beyond the example of a firm in its second period of life. The unrestricted history of estimated relative prices $\widetilde{r}_{i}^{t-1}$ and realized quantities forms the information set used to update beliefs about demand. Given past history $\widetilde{r}_{i}^{t-1}$, the worst-case beliefs feature kinks at all previously observed $\widetilde{r}_{i} \in \widetilde{r}_{i}^{t-1}$. When a review does not occur this period so that $\widetilde{p}_{j, t}=\widetilde{p}_{j, t-1}$, the kinks in expected demand occur at the same set of nominal prices as last period.

These kinks make stickiness in nominal prices akin to "price plans", where posted prices tend to bounce around a few "reference prices". However, unlike other frameworks such as Eichenbaum et al. (2011), price plans adjust gradually over time: as shocks push the firm to visit a price it has not posted previously, it is added to its "price plan". Importantly, in the future the firm is still likely to revisit the older prices, as the signals it has accumulated there

\footnotetext{
${ }^{16}$ Formally, these first-order losses dominate the standard markup and aggregate-demand effects of a change in $p_{t}$, as shown in Propositions A1 and A2, Online Appendix A.5. Naturally, we can modify either one of our two key primitives that (i) the economy is sub-divided in industries so that the set of firm's direct competitors is different from the set of all firms in the economy, and thus $p_{j, t} \neq p_{t}$, or that (ii) the firms are not confident that movements in $p_{t}$ translate one-to-one in movements in $p_{j, t}$, in ways that would recover full nominal flexibility. Online Appendix B.3 (on the authors' website) has details on such alternative economies.
} 
remain in its information set. As we discuss in Section 4.3, this feature has implications for the persistence of the real effects of monetary shocks.

\section{Quantitative evaluation}

Next, we evaluate quantitatively the empirical relevance of the model described in the previous section by testing its implications against a rich set of conditional and unconditional moments. This requires solving numerically the general infinite horizon decision problem of the ambiguity-averse firms. As discussed earlier, the dimensionality of the space grows with

the length of the history $\varepsilon^{t-1}$, and to handle this problem we use the same $\tilde{V}$ approximation as outlined in Section 2.4. The advantage of this approach is that we can leave $\varepsilon^{t-1}$ completely unrestricted, hence do not need to impose any ad-hoc assumptions limiting the memory of the firms. This way, we can evaluate the performance of our mechanism in the long-run, at the stochastic steady state of the model, where the history of observations $\varepsilon^{t-1}$ is both endogenous, reflecting past optimal choices, and long.

\subsection{Calibration}

The model period is a week. We calibrate $\beta=0.97^{(1 / 52)}$ to match an annual interest rate of $3 \%$. The mean growth rate of nominal spending $\mu=0.00046$ is set to match an annual inflation of $2.4 \%$, and we pick the standard deviation $\sigma_{s}=0.0015$ to generate an annual standard deviation of nominal GDP growth of 1.1\%. Following the calibration in Vavra (2014) we set the persistence and standard deviation of aggregate productivity $\rho_{a}=0.91^{(1 / 13)}=0.9928$ and $\sigma_{a}=0.0017$ to match the quarterly persistence and standard deviation of average labor productivity, as measured by non-farm business output per hour. We choose an elasticity of substitution of $b=6$, implying a (flexible price) markup of $20 \%$.

We choose the remaining parameters by targeting micro-level pricing moments from the IRI Academic Dataset. The dataset consists of scanner data for the 2001 to 2011 period collected from over 2,000 grocery stores and drugstores in 50 U.S. markets. The products cover a range of almost thirty categories, mainly food and personal care products. For our purposes, we focus on nine markets and six product categories. ${ }^{17}$ Because our model does not feature a rationale for sales, all reported moments are based on "regular price" series in

\footnotetext{
${ }^{17}$ The markets are Atlanta, Boston, Chicago, Dallas, Houston, Los Angeles, New York City, Philadelphia and San Francisco. The categories are beer, cold cereal, frozen dinner entrees, frozen pizza, salted snacks and yogurt. A more complete description of the dataset is available in Bronnenberg et al. (2008).
} 
which temporary sales are filtered out. ${ }^{18}$

\section{Learning parameters and stochastic shocks}

Our mechanism emphasizes non-parametric learning under ambiguity, which creates a rich learning environment characterized by six parameters $\left\{\delta, \gamma_{p}, \gamma, \sigma_{x}^{2}, \psi, \lambda_{T}\right\}$. With a focus on limiting the associated degrees of freedom, we set two of the learning parameters to values corresponding to natural limiting cases, and freely estimate the remaining four parameters.

First, regarding ambiguity over the demand function, we assume that the firm is confident that the mean demand function cannot be locally upward sloping, hence $\delta \leq b$. To minimize degrees of freedom, we thus simply fix $\delta=b$. Second, in terms of ambiguity over the unobserved industry price index, the parameter $\gamma_{p}$ controls the size of the entertained set of cointegration relationships in equation (17). As detailed in Section 3, a positive $\gamma_{p}$ is the reason why the joint worst-case beliefs about the demand function and the relative price lead to nominal rigidity in the short run. However, once the worst-case is determined and the firm engages in learning through the perceived relative price $\widetilde{r}_{i}$, the value of $\gamma_{p}$ only enters as a price-independent demand shifter in the worst-case expectation. Its quantitative role is therefore limited and thus we study the limit of $\gamma_{p} \rightarrow 0$. This leaves four learning parameters $\left\{\gamma, \sigma_{x}^{2}, \psi, \lambda_{T}\right\}$ that we estimate by targeting micro-level moments, as detailed below.

The only modeling difference relative to the environment described in Section 3 is the assumption that with probability $\lambda_{\phi}$, firm $i$ exits and a newly-born firm takes its place in industry $j$. New firms have no information on the demand function beyond the timezero prior, thus exit resets the information capital of firms. ${ }^{19}$ This assumption serves two purposes. First, with an infinitely growing history of signals, conditional beliefs are nonstationary, making it difficult to evaluate behavior at the stochastic steady state. Second, it allows us to study pricing behavior over the firm's life-cycle, which serves as an additional set of untargeted moment restrictions on our learning mechanism. Here, we set the exit probability $\lambda_{\phi}=0.0075$, following Argente and Yeh (2017), who provide a detailed analysis of the duration of a UPC-store pair in the same IRI dataset that we use.

The firm's quantity sold is subject to demand shocks, with a standard deviation of $\sigma_{z}$. We calibrate this parameter by using empirical evidence on the accuracy of predicting one-

\footnotetext{
${ }^{18}$ We use the methodology of Nakamura and Steinsson (2008) which aims to eliminate V-shaped sales. Also, as is usual with scanner datasets, we obtain the unit price by dividing weekly revenue by quantity sold. In order to minimize the probability that we identify spurious price changes due to middle-of-theweek repricing, the use of coupons, loyalty cards, etc., we take the conservative approach of eliminating any observations that feature a price with fractional cents.

${ }^{19}$ As such, we interpret reseting the informational capital as a broad concept, which includes any shock that makes the firm unsure that past observations are still informative, including major changes in the competitive landscape, the introduction of rival substitutes or technological change.
} 
period-ahead quantity. This involves estimating the demand regression:

$$
q_{i j t}=\beta_{0}+\beta_{1} q_{i, j, t-1}+\beta_{2} p_{i j t}+\beta_{3} p_{i j t}^{2}+\beta_{4} c p i_{t}+\text { week }_{t}^{\prime} \theta_{1}+\text { store }_{j}^{\prime} \theta_{2}+i t e m_{i}^{\prime} \theta_{3}+z_{i j t}
$$

where $q_{i j t}$ and $p_{i j t}$ are quantities and prices in $\operatorname{logs}$ for item $i$ in store $j$ at time $t$; $c p i_{t}$ is the (log) consumer price index for food and beverages; while $w_{e e} k_{t}$, store $_{j}$ and item $_{i}$ are vectors of week, store and item dummies respectively. ${ }^{20}$ We then compute the empirical standard deviation of the residuals $z_{i j t}$ leading us to set $\sigma_{z}=0.613 .^{21}$

The firms also face idiosyncratic productivity shocks, whose persistence and volatility (respectively $\rho_{w}$ and $\sigma_{w}$ ) we estimate via moment matching.

\section{Simulated method of moments}

We estimate the six free parameters, $\left\{\rho_{w}, \sigma_{w}, \sigma_{x}, \psi, \lambda_{T}, \gamma\right\}$, via simulated method of moments, by targeting the six pricing moments listed in Table II. For the most part, these are basic pricing moments widely used in the literature to discipline price-setting models. Throughout, we define the 'reference price' as the modal price within a 13-week window period, as in Gagnon et al. (2012). The last moment, the mean duration of a pricing regime, appeals to the fact that in our model, the kinks in expected demand turn basic stickiness into price plans. In both actual and simulated data, we identify these price plans using the method in Stevens (2014). ${ }^{22}$ Table I presents all parameters values, while Table II shows the outcomes for moments targeted in the estimation. ${ }^{23}$ The model matches the targeted moments very well and, naturally, it does so through a positive ambiguity parameter $\gamma$, which is the necessary source for any price stickiness in the model. ${ }^{24}$

\subsection{Testable implications}

Next, we analyze the ability of the model to match various features of the data that were not directly targeted in the estimation, yet speak to the mechanisms at the heart of our model.

\footnotetext{
${ }^{20}$ Given the high (weekly) frequency of our data and the fact that we do not find evidence of middle-ofthe-week price changes, endogeneity is unlikely to be a significant issue here.

${ }^{21}$ The regression is run and the volatility measure is computed first for each of the 54 category/market pairs, before being aggregated using revenue weights.

${ }^{22}$ The methodology modifies the Kolmogorov-Smirnov test to identify shifts in the distribution of price changes over time. In order to have enough observations from which to identify regimes when applying to the data, we ignore quote-lines that have missing price data or less than 104 weekly observations (2 years). We use Stevens (2014)' standard critical value of 0.61 throughout our regime identification exercises, for both actual and simulated data. Also, in both cases, we eliminate regular price changes of less than $1 \%$.

${ }^{23}$ The estimation is based on a simulated panel of 5000 time periods with 1000 active firms in each period.

${ }^{24}$ Online Appendix A.6 shows that the estimated $\gamma$ implies an empirically plausible amount of ambiguity, as it generates dispersion in prior demand forecasts that matches the evidence in Gaur et al. (2007).
} 
Table I. Parameter Values

\begin{tabular}{lllllll|llllll}
\hline \hline \multicolumn{4}{c}{ Calibrated Parameters } & \multicolumn{5}{c}{ Estimated Parameters } \\
\hline \hline$\beta$ & $\mu_{s}$ & $\sigma_{s}$ & $\rho_{a}$ & $\sigma_{a}$ & $\sigma_{z}$ & $\lambda_{\phi}$ & $\rho_{w}$ & $\sigma_{w}$ & $\sigma_{x}$ & $\psi$ & $\lambda_{T}$ & $\gamma$ \\
\hline 0.9994 & 0.00046 & 0.0015 & 0.993 & 0.0017 & 0.613 & 0.0075 & 0.998 & 0.008 & 0.691 & 4.609 & 0.018 & 0.614
\end{tabular}

All model moments are computed at the stochastic steady state. An important characteristic of the steady state is that the typical information set of a firm turns out to feature demand observations at a relatively small number of distinct price points. Because of this endogenous sparsity of the information set, the typical firm has several price points associated with low uncertainty that are both sticky and likely to be revisited, but also still faces substantial residual demand uncertainty away from those price points. Online Appendix A.8 provides a detailed discussion of the typical information set at the stochastic steady state.

We start by covering moments that have previously been analyzed in the literature, before turning to more novel features. The results are presented in Table III. All empirical moments are obtained by weighing results across markets and categories.

Table II. Targeted moments - Data vs model

\begin{tabular}{lll}
\hline \hline & Data & Model \\
\hline Frequency of regular price changes & 0.107 & 0.105 \\
Median size of absolute regular price changes & 0.149 & 0.154 \\
75th pctile of the distribution of non-zero absolute price changes & 0.274 & 0.277 \\
Fraction of non-zero price changes that are increases & 0.535 & 0.533 \\
Frequency of modal price changes (13-week window) & 0.027 & 0.026 \\
Mean duration of pricing regimes & 29.90 & 30.54 \\
\hline \hline
\end{tabular}

Table III. Untargeted moments - Data vs model

\begin{tabular}{llll}
\hline \hline & & Data & Model \\
\hline Panel A & Prob. modal $P$ is max $P$ & 0.819 & 0.740 \\
& Fraction of weeks at modal $P$ (13-week window) & 0.829 & 0.880 \\
& Prob. price moves to modal $P$ & 0.592 & 0.646 \\
\hline Panel B & Prob. revisiting old price $(26$-week window) & 0.478 & 0.493 \\
& uni (26-week window) & 0.774 & 0.822 \\
\hline Panel C & Avg hazard slope & -0.011 & -0.015 \\
\hline Panel D & Kurtosis( $\Delta P$ distribution) & 3.00 & 2.16 \\
& Prob. modal P revisits old modal P (52-week window) & 0.085 & 0.112 \\
& Prob. revisiting price from before last modal P change & 0.269 & 0.253 \\
& Prob. revisiting price from before brand new price & 0.327 & 0.387 \\
\hline \hline
\end{tabular}




\subsubsection{Reference prices, memory and size distribution}

We first show that our model matches well a number of moments analyzed in the literature.

Reference prices. Panel A of Table III shows that the model correctly predicts that the typical modal price is generally also the highest price in a given 13-week window - the probability of that occurring in the data is $82 \%$ vs. $74 \%$ in the model. Second, within each 13-week window, we also compute the average fraction of weeks that the regular price spends at the reference (modal) price: while in the data the regular price spends $83 \%$ of the time at the modal price, in the model this fraction equals $88 \%$. We confirm that this is not simply a by-product of pervasive price stickiness: the probability that a non-modal regular price change ends at the modal price, and not at some other regular price, is $65 \%$ in the simulations compared to $59 \%$ in the data.

Discreteness and memory. In our model, the first-order perceived cost of moving away from any of the previously-observed prices implies that prices display memory (see Corollary 3). Panel B of Table III reports the probability that, conditional on a price change, the firm posts a regular price that it has already visited within the last six months (26 weeks): this probability is $49 \%$ in our model, and $48 \%$ in the data. Note that a standard menu cost or Calvo model would feature no such price memory and the probability would be $0 \%$.

A related empirical observation is that firms tend to cycle through a relatively limited, discrete set of prices as opposed to posting a lot of new unique prices. To test this property, we produce a novel statistic that takes into account price stickiness: for each product $i$ (a given UPC) sold in a specific store $j$, we compute the number of unique prices and price changes observed within the 26-week window centered around week $t$, and denote them by $u_{i j t}$ and $c_{i j t}$ respectively. We then define the ratio $u n i_{i j t} \equiv u_{i j t} /\left(c_{i j t}+1\right){ }^{25}$ Note that if all price changes end up at a price that had not been visited before within a specific window, the ratio $u n i_{i j t}$ is equal to 1 . Yet, in the data we see that the average value of this moment is 0.774 , as reported in Table III. This is very close to the simulated moment of 0.822 .

Size distribution of price changes In our model, the perceived cost of changing prices is history dependent and a function of the absolute size of the price change. As a result, our model allows for the co-existence of large and small price changes. This property is evident from Figure 3, which plots the distribution of the size of price changes in the model (left

\footnotetext{
${ }^{25}$ In both the data and the model, we drop from the computation any window that features no price change. We thank an anonymous referee for suggesting this moment to us.
} 
panel) and the data (right panel). While parameter heterogeneity across firms would allow for a smoother distribution, our model clearly can generate price changes of various sizes.
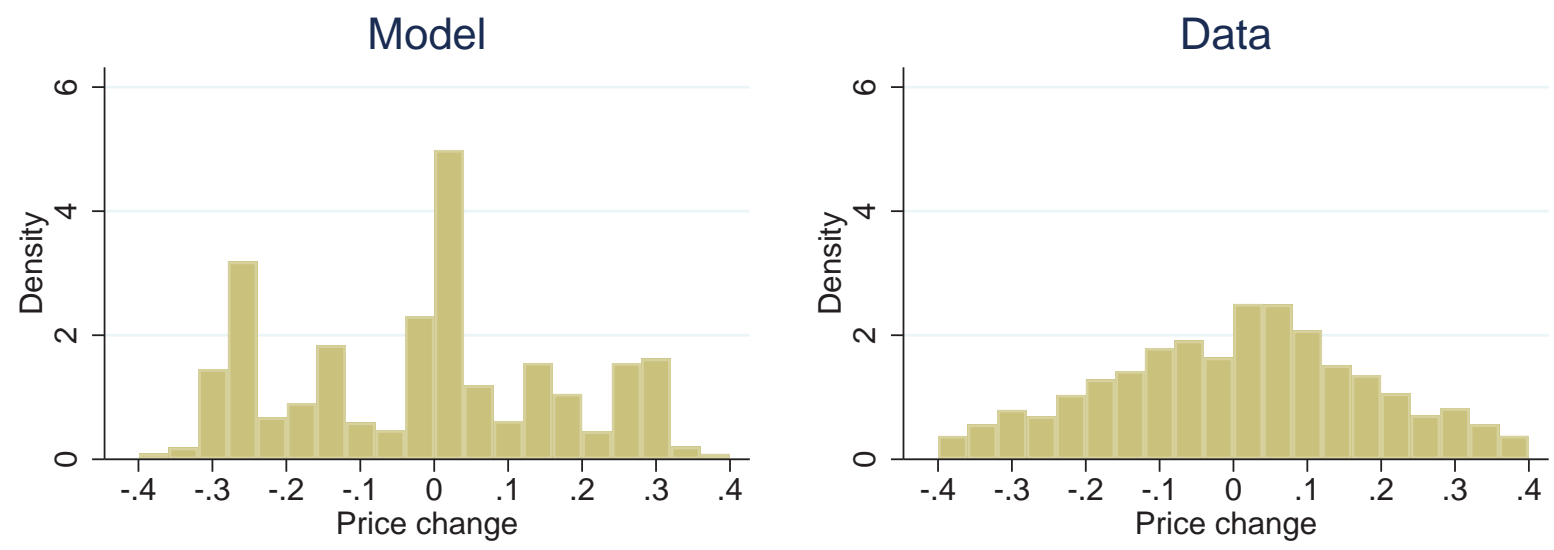

Figure 3. Distribution of the absolute size of price changes. Data vs. simulations.

\subsubsection{Hazard function of price changes}

An important force in our setup is that, all else equal, a firm is less willing to move away from a price that it has stayed at for longer and thus acquired more information about. This naturally gives rise to a declining hazard function of price changes: the probability of a price change conditional on the price having survived $\tau$ periods is decreasing in $\tau$.

The shape of the hazard function has been heavily discussed in the price-setting literature. Nakamura and Steinsson (2008), for example, estimate a downward-sloping hazard using U.S. CPI data, a characteristic that they consider represents a challenge to many popular pricesetting mechanisms. Some, however, have argued that this finding could be a by-product of heterogeneity: as noted by Klenow and Kryvtsov (2008), "[t]he declining pooled hazards could simply reflect a mix of heterogeneous flat hazards, that is, survivor bias."

In light of this word of caution, our approach is to employ a linear probability model (LPM) with a rich set of fixed effects to control for heterogeneity in unconditional price change frequencies that may mechanically generate downward-sloping hazard functions. The linear regression circumvents the incidental parameters problem that arises with the use of fixed effects in non-linear models, such as a proportional hazard framework or a probit. ${ }^{26}$

For each category/market, we run a separate regression of the type:

$$
\mathbb{1}\left(p_{i, j, t} \neq p_{i, j, t-1}\right)=\alpha+\beta \tau_{i, j, t}+\gamma_{i}+\gamma_{j}+\gamma_{t}+u_{i, j, t}
$$

\footnotetext{
${ }^{26}$ In Online Appendix B.4 (on the authors' website), we apply our econometric approach to panels of simulated data and show that it allows us to recover the true value of the slope of the hazard function, even in the presence of pervasive heterogeneity.
} 
where the symbol $\mathbb{1}($.$) denotes the indicator function. Since \tau_{i, j, t}$ is the length of the price spell (i.e. the number of weeks since the price has last been changed), the coefficient $\beta$ therefore represents the estimate of the slope of the hazard function. Finally, $\gamma_{i}, \gamma_{j}$ and $\gamma_{t}$ are product, store and week fixed effects respectively. These shifters control for any systematic heterogeneity that would bias downwards the slope of the hazard. We run the regression on spells of 26 weeks or less, where the vast majority of observations lie.

Panel C of Table III shows that we obtain a slope estimate $\widehat{\beta}$ of -0.011 , once averaged across the 54 category/market pairs. This value implies that each additional week that a spell survives lowers the probability of observing a price change by about 1.1 percentage point. The estimated slope coefficients are negative and statistically significant at the $1 \%$ level in all category/market pairs, whether we use unweighted or weighted observations. ${ }^{27}$

To evaluate the model's ability to match the empirical hazard, we estimate the same LPM regression on the data simulated by the model. At -0.015, the slope of the simulated hazard is steeper, yet compares well with its empirical counterpart.

In Online Appendix A.9 we apply the alternative approach of Campbell and Eden (2014) and reach the same conclusion: the hazard of regular price changes is downward sloping and of similar magnitude in both model and data.

\subsubsection{Pricing behavior over the product life-cycle}

Using the same dataset as ours, Argente and Yeh (2017) find that both the frequency and size of price changes decline significantly with the age of the typical the product. In our model, the price behavior over the life-cycle of the product/firm is shaped by the history dependence of the optimal pricing decision through the interaction of two forces. First, at the beginning of its life, the firm does not have much information about the demand curve of the product it sells and has therefore not yet established any deep perceived kink in expected demand. Second, the fact that the firm has very little information about demand increases the relative value of experimentation, as discussed in Section 2.4. Both of these forces imply that price flexibility decreases with age: newly-born firms tend to change prices more frequently than firms that have been in existence for a while and have accumulated significant information capital at past prices. Similarly, the experimentation motive implies that the average size of price changes for young firms is larger than that of older firms.

We quantify the life-cycle properties of the frequency and size of price changes in our

\footnotetext{
${ }^{27}$ We cluster standard errors at the store level, the cluster which yields the highest standard errors. Also, in line with the literature, we drop all left-censored spells from the sample. Lastly, in Figure B.1 of the Online Appendix B.5 (on the authors' website), we plot the distribution of coefficient estimates $\widehat{\beta}$ across the 54 category/market pairs.
} 
model by running the following two regressions on the simulated data:

$$
\begin{gathered}
\mathbb{1}\left(p_{i, t} \neq p_{i, t-1}\right)=\beta_{0}^{\text {freq }}+\beta_{1}^{\text {freq }} \mathbb{1}\left(\text { age }_{i, t} \leq 26\right)+\varepsilon_{i, t} \\
\left|\Delta p_{i t}\right|=\beta_{0}^{\text {size }}+\beta_{1}^{\text {size }} \mathbb{1}\left(a g e_{i, t} \leq 26\right)+\varepsilon_{i, t},
\end{gathered}
$$

where the coefficients of interest are $\beta_{1}^{\text {freq }}$ and $\beta_{1}^{\text {size }}$, which capture respectively the frequency and size of price changes in the first 6 months of a firm's life relative to the next half a year. ${ }^{28}$

In both cases, we find positive and statistically-significant coefficients: $\widehat{\beta}_{1}^{\text {freq }}=0.23$ and $\widehat{\beta}_{1}^{\text {size }}=0.09$. In other words, our model predicts that both the frequency and size of price changes fall as a new product ages, in line with the evidence from Argente and Yeh (2017).

\subsubsection{Past demand realizations and price-setting decisions}

The focus so far has been on price-related moments, as is common in the literature. Yet, our model also has stark and unique implications about the relationship between quantities and prices. In particular, the perceived cost of changing the last posted price increases with the realized value of the demand shock at that price (see Result 4): a firm that observes a particularly good demand realization is more likely to stay put, while bad demand realizations raise the likelihood of a price reset.

To test this prediction, we first extract demand innovations in the data by using regression (31), which was described earlier. The object of interest is the residual $z_{i j t}$, the unexplained or "surprise" demand component for item $i$ in store $j$ at time $t$. We then construct two indices that capture how attractive a given price may be from the perspective of the firm. We define the $z$-score of price $p_{i j t}$ as:

$$
\text { zscore }_{i j t}=\frac{\sum_{\tau=0}^{26}\left[\text { valid }_{i j, t-\tau} \times z_{i j, t-\tau}\right]}{\sum_{\tau=0}^{26} \text { valid }_{i j, t-\tau}} .
$$

The indicator valid $_{i j, t-\tau}=1$ if $p_{i j t}=p_{i j, t-\tau}$, that is, if the price at time $t-\tau$ is the same as the one we compute the $z$-score for. Conceptually, the $z$-score of price $p_{i j t}$ corresponds to the average of the demand innovations at that price. ${ }^{29}$ It is also useful to define a version of the zscore that only incorporates demand innovations up to $t-1$, and which therefore

\footnotetext{
${ }^{28}$ Focusing on first 12 months of life helps isolate the life-cycle effects. The estimates are even more pronounced if we do not censor on the right. As is typical with any moments on the size of price changes, the second regression only considers time periods with a non-zero price change.

${ }^{29}$ We truncate the window to 26 weeks to capture the idea that demand realizations very far back are likely to be of little value to the firm. We also tried to geometrically discount past observations; this has little impact on the results.
} 
informs the price choice at $t$ :

$$
\text { score }_{i j t}^{l a g}=\frac{\sum_{\tau=1}^{26}\left[\text { valid }_{i j, t-\tau} \times z_{i j, t-\tau}\right]}{\sum_{\tau=1}^{26} \text { valid }_{i j, t-\tau}} .
$$

Finally, we define $w_{s c o r} e_{i j t}$, which captures how often a price has been posted in the past:

$$
\text { wscore }_{i j t}=\sum_{\tau=0}^{26} \operatorname{valid}_{i j, t-\tau} .
$$

In order to test whether the firm is less (more) likely to move away from a price that experienced an unexpectedly good (bad) demand realization, we run the following regression:

$$
\mathbb{1}\left(p_{i, j, t} \neq p_{i, j, t-1}\right)=\beta_{0}+\beta_{1}\left(\text { zscore }_{i j, t-1}-\text { zscore }_{i j, t-1}^{l a g}\right)+\beta_{2} \text { wscore }_{i j, t-1}+f_{i j}+\varepsilon_{i j t} .
$$

The LHS equals 1 when the price at $t$ is different than at $t-1$, and 0 otherwise. The regressor of interest, $z s c o r e_{i j, t-1}-z s c o r e_{i j, t-1}^{l a g}$, corresponds to the change in the $z$-score of the price posted at $t-1$ : a positive value indicates that all else equal, the firm was hit by a relatively good demand realization at time $t-1 .^{30}$ We also control for the $w$-score, which is the proper way of controlling for the declining hazard under the null hypothesis of our mechanism. When run on the actual data, the panel regression includes either category/market or product/store fixed effects $f_{i j}$ in order to control for the heterogeneity in price change frequency.

For both the model and the data, we run two main regressions. The first one imposes no additional restrictions. The second uses only observations for which sscore $_{i j, t-1} \leq 12$, so that the price the firm is considering leaving has been posted for at most half of the periods within the backward-looking 26-week window. This distinction is driven by our model prediction that new demand realizations are less likely to influence the decision to change a price that has been observed more often in the past (high $w$-score).

Table IV presents the results of running the regression in equation (33) on both the actual and simulated data. To ease the interpretation, the coefficients are reported as marginal effects: the impact of a one-standard-deviation deviation in the $z$ - or $w$-score on the likelihood of a price change. All coefficients are statistically significant at the $1 \%$ level. Three observations on the $z$-score effect are worth highlighting.

First, the effect is negative in all regressions: a good (bad) demand realization at the posted price that lifts (lowers) the $z$-score decreases (increases) the chance of moving away from that price. This is in contrast to most state-dependent mechanisms, such as a standard

\footnotetext{
${ }^{30}$ To minimize the risk that changes in the $z$-score are driven by some complex non-linearity in the demand function, we focus on observations for which there was no price change at $t-1$, i.e. $p_{i, j, t-1}=p_{i, j, t-2}$.
} 
menu-cost model: in these environments, both positive and negative shocks make the firm more likely to reprice as they raise the gap between the current and optimal prices.

Second, the effect is indeed larger for more "recent" prices (low $w$-score): while a onestandard-deviation change in the $z$-score decreases the probability of a price change by between 80 to 90 basis points when we condition on sscore $_{i j, t-1} \leq 12$, the effect is only around 55 basis points with wscore $_{i j, t-1} \leq 25$. The effects are also economically meaningful, as a $80 \mathrm{bp}$ increase in the probability of a price change is about $10 \%$ of the unconditional probability of a price change in the data.

Third, the $z$-score effects in the data and the model are similar: for younger prices, the absolute impact on the price change frequency is $83 \mathrm{bp}$, almost perfectly in line with the 86$87 \mathrm{bp}$ effect in the data. They also compare favorably when conditioning on $w_{s c o r e} e_{i j, t-1} \leq 25$ (65bp vs. $57-58 b p$ in the data).

Table IV. Results from the $z$-score regressions

\begin{tabular}{lcccccc}
\hline \hline & \multicolumn{3}{c}{ Data } & \multicolumn{3}{c}{ Model } \\
wscore $_{i j, t-1} \leq x$ & \multicolumn{1}{c}{$x=12$} & $x=25$ & $x=12$ & $x=25$ \\
\hline score $_{i j, t-1}-z$ score $_{i j, t-1}^{\text {lag }}$ & -0.0087 & -0.0086 & -0.0058 & -0.0057 & -0.0083 & -0.0065 \\
wscore $_{i j, t-1}$ & -0.0373 & -0.0290 & -0.0466 & -0.0264 & -0.0253 & -0.0195 \\
\hline $\begin{array}{l}\text { Category/market FE } \\
\text { Product/store FE }\end{array}$ & $\mathrm{X}$ & $\mathrm{X}$ & $\mathrm{X}$ & $\mathrm{X}$ & & \\
\hline \hline
\end{tabular}

Note: The dependent variable equals 1 when $p_{i, j, t} \neq p_{i, j, t-1}, 0$ otherwise. The empirical regressions include either both category and market fixed effects, or item/store fixed effects. We report marginal effects: the impact of a one-standard-deviation in the independent variable on the likelihood of a price change. Standard errors are clustered at the category-market level. All coefficients are statistically significant at $1 \%$ level.

\subsection{Monetary non-neutrality}

In this section, we argue that our theory is relevant not only due to its successful micro-level predictions, but also because the model alters the standard relationships highlighted in the literature between micro-level moments and the propagation of nominal shocks.

We quantify the degree of monetary non-neutrality by computing the impulse response of total output produced by the measure-zero set of ambiguity-averse firms to an innovation in aggregate nominal spending. Note that because all other firms have rational expectations, our exercise arguably represents a lower bound on the size and persistence of monetary non-neutrality since it ignores any strategic complementaries in price setting. 


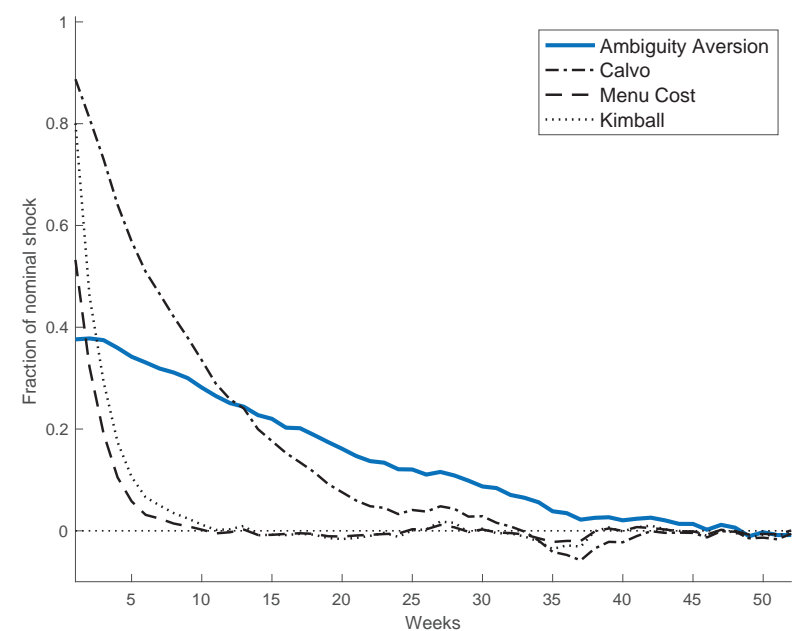

(a) Matching regular price change frequency

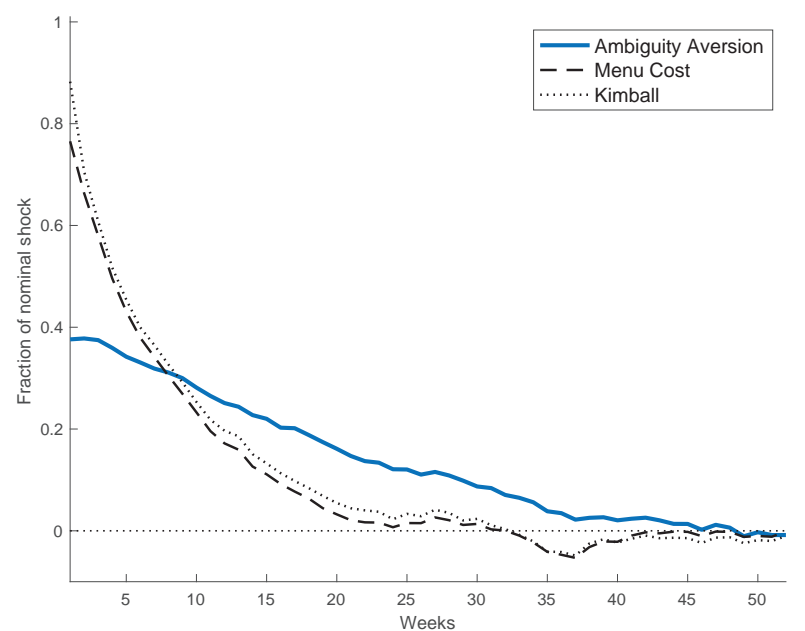

(b) Matching reference price change frequency

Figure 4. Nominal spending effects on real output.

We estimate the impulse responses via Jordá (2005) projections, an approach well suited to the high degree of non-linearity in our model. In the expression below, we regress the $t+k$ output of ambiguity-averse firms on the nominal shock $\epsilon_{t}^{s}$

$$
\ln \left(\int Y_{i, t+k} d i\right)=\alpha_{k}+\beta_{k} \epsilon_{t}^{s}+u_{j, t+k} .
$$

The coefficients $\beta_{k}$ represent the impulse response of output to the nominal shock, plotted by the solid line in Figure 4, panel (a). The response is shown as a fraction of the shock. We find that, for a $1 \%$ shock, real output increases by $0.38 \%$ on impact, and this increase is persistent, with full a cumulative output effect of $7.2 \%$ after 52 weeks.

\section{Alternative models}

To provide context for our results, we contrast our mechanism with three simple and widely used alternative mechanisms of nominal rigidity. ${ }^{31}$ The first is a Calvo model, where the firm can change its price with an exogenous probability. The second is a menu cost version, where the firm can change its price at any time by paying a fixed cost. The third model uses Kimball (1995) preferences which create a smoothed version of a kink in demand - a form of real rigidity. To generate nominal rigidity in that model, we follow the literature and assume

\footnotetext{
${ }^{31}$ Online Appendix B.7 (on the authors' website) details instead comparative statics within our model, focusing on the resulting nuanced link between price flexibility, memory, and non-neutrality.
} 
that the firm must pay a small menu cost. ${ }^{32}$ Besides these differences, all specifications share the same economic framework: we study a similar measure-zero sector and assume the same cost processes as in our benchmark setup.

The Kimball model represents a particularly useful comparison because even if it shares a similar kinked-demand flavor, the underlying mechanism is very different from ours. For one, our framework generates stickiness without additional fixed costs since it features kinks that are not smoothed-out. Second, our model generates perceived kinks in demand; in a Kimball world, an econometrician would be expected to find evidence of actual kinks in demand schedules. This property is not innocuous as it has proven difficult to find evidence for the large super-elasticity values that are needed to jointly generate a significant persistence of monetary shocks and plausible micro price facts (see the evidence in Dossche et al. (2010) and the analysis in Klenow and Willis (2016)).

Our model of rigidity is also consistent with a range of facts, such as experimentation and effects of the level of past demand realizations on price setting, that are driven by our learning forces which are absent in the three considered alternative models. A crucial property here is that in the alternative models, conditional on a price change the gap towards the new optimal frictionless price is perfectly closed and the probability that the firm visits an old price is zero. Instead, in our framework, the firm does not typically eliminate this gap, preferring to return to a previously visited "safe" price due to ambiguity aversion. As we discuss below, this type of price memory has important implications for monetary non-neutrality.

Our first set of comparisons are based on calibrating the respective free parameter of each of the alternative models (Calvo probability or menu cost) to the same $10.5 \%$ frequency of regular price changes as in our ambiguity model. The impulse responses corresponding to these models are marked by the non-solid lines in Figure 4, panel (a). Overall, the cumulative real effect in our model is similar to that of the Calvo model $(7.58 \%)$, and significantly larger than in the menu cost and Kimball models (1.12\% and $1.81 \%$ respectively).

\section{Impulse responses}

The most striking difference is in the degree of persistence. In our model, the real effect only dies out after 48 weeks and has a half-life of 18 weeks. In contrast, in the menu cost and Kimball models the real effects have half-lives of less than 3 weeks, and disappears within 10 weeks, while in Calvo it has a half-life of 8 weeks and dies out 32 weeks in. The different persistence across these standard models is due to the Golosov and Lucas (2007) selection effect - the adjusting firms in the Calvo framework are chosen randomly, but are

\footnotetext{
${ }^{32}$ See for example Klenow and Willis (2016), whom we follow to use a value of 10 for the demand superelasticity (elasticity of the demand's price elasticity) in the Kimball aggregator.
} 
self-selected in the two other versions and hence adjust by a lot on average, leading to a quick transmission of the shock.

The fundamental reason for the significantly larger persistence in our model is that, consistent with the data, it features memory in nominal prices: a substantial proportion of price movements occurs between perceived demand kinks that have formed prior to the shock. As the firm revisits old price points, aggregate adjustment is slowed down, even though prices look very flexible at the micro level. Indeed, our environment features something akin to a "price plan" - a collection of low-uncertainty prices that the firm has visited in the past and switches between relatively flexibly.

This flexibility when switching between low-uncertainty, previously-visited prices accounts for the muted real effect on impact of the nominal spending shock. Intuitively, as noted by Alvarez and Lippi (2019), in models that have price plan-like behavior (like ours) firms actively use their free but imperfect margin of adjustment, leading to significant flexibility on impact. Yet, in our model, the blunted real effect on impact is more than made up for by the high persistence, leading to a large cumulative real effect, as well as to impulse response dynamics that are not well approximated by any of the three alternative models.

\section{Frequency and kurtosis of regular price changes}

Alvarez et al. (2016) show that the cumulative degree of nominal non-neutrality in a wide range of models, including the three alternative frameworks we study, is proportional to the kurtosis of the regular price change distribution. Intuitively, this proportionality arises because in this class of models the derivative of the density of price gaps at the adjustment thresholds, and implicitly the Golosov and Lucas (2007) selection effect, becomes small only for sufficiently leptokurtic distributions of price changes.

In contrast, as noted by Alvarez and Lippi (2019), frameworks that feature price plans, like ours, do not fit the class of models analyzed in Alvarez et al. (2016). Indeed, we find that our mechanism does not abide by this sufficient statistic relationship, in a manner that is quantitatively important. As we report in Panel D of Table III, the distribution of the regular price changes has a kurtosis of 3 in the data, while our model generates a kurtosis of 2.16. The standard analysis would predict that such a model-implied kurtosis, if anything an underprediction of its empirical value, would be associated with weak real effects of nominal shocks. However, even though the kurtosis in our model is significantly lower than in the Calvo version, (2.16 versus 5.7), the cumulative real effects are very similar $(7.2 \%$ versus $7.6 \%)$. In fact, our kurtosis is much closer to that of the menu cost and Kimball models, at 1.23 and 1.79 respectively, yet our model implies a cumulative real effect that is six times larger than in the menu cost model. 
The broad observation that price-plan-like behavior is important for mapping moments of regular price changes to the propagation of nominal shocks is not unique to our model - see previous discussion in Eichenbaum et al. (2011) (EJR) and Kehoe and Midrigan (2015). Yet, we argue next that the nature of price memory in our model is fundamentally different than in existing frameworks, in a way that has important implications for aggregate dynamics.

\section{Gradual adjustments in price plans}

If our mechanism was simply an endogenous version of the EJR "price plan", then the persistence of the real output effect to a money shock would be governed by the frequency of changes in reference prices. In particular, Alvarez and Lippi (2019) show that a simple menu cost model calibrated to the frequency of reference price changes provides a useful upper bound on the effect of such a "price plan" model. But that is not true for our model - panel (b) of Figure 4 shows that our model's real effects are significantly more persistent and as a result cumulatively larger ( $7.2 \%$ versus $5.5 \%$ and $6 \%$, respectively) than those from a menu cost or Kimball model matched to the same frequency of reference price changes.

The reason is that the price plans in our model adjust gradually. In a standard price-plan model like EJR, once a firm decides to update its plan, it resets all prices within the plan. In our model, instead, the price plans evolve slowly, with new prices being added to the effective plan as the firm experiments with and learns about demand at new price points. The reason is that when shocks drive a firm to post a price outside of the set it has been visiting in the past, this does not destroy the information capital it has built up at its old price points. Hence, when idiosyncratic shocks mean-revert, the firm is likely to revisit those old price points. This is important: the fact that the firm returns to known prices, even after sampling a new portion of the price space, is what slows down the evolution of the aggregate price level beyond what is captured by the frequency of reference price changes.

We quantify the importance of these gradual price-plan adjustments in both the model and the data in three ways, which we report in Panel D of Table III. First, we consider memory in reference, as opposed to regular, prices: we compute the probability that a change in the reference price (i.e. the modal price in a 13-week window) revisits one of the old reference prices set in the past 52 weeks. The previous literature has not studied potential memory in reference prices; under the implicit assumption that reference price changes capture price plan resets, such as in EJR, such memory should be zero. Instead, we

find that these probabilities are $8.5 \%$ and $11.2 \%$ in the data and model respectively, pointing at significant memory in reference prices.

Next, we try to identify gradual price plan changes. Consider that a reference price change occurs at time $t$. We compute the probability that the first price reset following the 
reference price change leads to a price that had already been posted before period $t$. In standard memory models such as EJR, this probability would be $0 \%$, as firms reset their whole price plan at once. Yet, we find this probability to be $27 \%$ in the data, compared to $26 \%$ time in the model. We also consider an alternative approach to capturing partial price plan changes. We first identify occurrences of "brand new prices", which are defined as prices that have not been posted in the previous 52 weeks. We then compute the probability that the first price change that follows a time- $t$ brand new price revisits a price that was posted before $t$ (ostensibly from before the shift in the "price plan"). This probability is equal to $33 \%$ in the data, and $39 \%$ in the model.

\section{Summary}

Overall, our theory offers a micro-foundation for price memory that delivers novel implications at both the micro and macro levels. First, we show that our unified theory of rigidity and memory jointly matches a host of over-identifying restrictions on microlevel pricing behavior. These additional moments not only provide external validity of the underlying theory, but also imply a more nuanced picture of how the observed microlevel price flexibility maps into the aggregate propagation of nominal shocks. Second, our framework endogenously generates slow and gradually adjusting price plans. This feature implies much more persistent real effects from monetary shocks than in a benchmark menu cost model calibrated instead to the frequency of reference price changes. As a result, we believe that our mechanism may be better suited at capturing the high persistence of monetary policy effects estimated in the data (Christiano et al. (2005)).

\section{Conclusion}

In this paper we show how firms' specification doubts about their perceived model of demand leads to a novel theory of price stickiness. We find strong empirical support for our theory by subjecting the mechanism to a rich set of micro-level implications. The parsimony and quantitative relevance of the mechanism make it a promising step towards building macroeconomic models that can be used for counterfactual analysis. Importantly, the theory has novel predictions about the way in which nominal shocks affect the aggregate economy.

\section{References}

Alvarez, F., H. Le Bihan, And F. Lippi (2016): "The real effects of monetary shocks in sticky price models: a sufficient statistic approach," American Economic Review, 106, 
$2817-51$.

Alvarez, F. And F. Lippi (2019): "Temporary Price Changes, Inflation Regimes and the Propagation of Monetary Shocks," American Economic Journal: Macroeconomics, forthcoming.

Argente, D. And C. Yeh (2017): "Product's Life Cycle, Learning, and Nominal Shocks," Manuscript, Minneapolis Fed.

Bachmann, R. And G. Moscarini (2011): "Business cycles and endogenous uncertainty," Manuscript, Yale University.

Baley, I. AND J. A. Blanco (2018): "Firm uncertainty cycles and the propagation of nominal shocks," AEJ: Macroeconomics, forthcoming.

BAll, L. AND D. Romer (1990): "Real rigidities and the non-neutrality of money," The Review of Economic Studies, 57, 183-203.

Bergemann, D. And K. Schlag (2011): "Robust monopoly pricing," Journal of Economic Theory, 146, 2527-2543.

Bergemann, D. And J. Valimaki (2008): "Bandit problems," The New Palgrave Dictionary of Economics, 2nd ed. Macmillan Press.

Bils, M. And P. Klenow (2004): "Some evidence on the importance of sticky prices," Journal of Political Economy, 112, 947-985.

Bonomo, M. and C. Carvalho (2004): "Endogenous time-dependent rules and inflation inertia," Journal of Money, Credit and Banking, 1015-1041.

BronnenberG, B., M. Kruger, And C. Mela (2008): "Database paper: The IRI Marketing Data Set," Marketing Science, 27, 745-748.

Campbell, J. R. And B. Eden (2014): "Rigid prices: Evidence from US scanner data," International Economic Review, 55, 423-442.

Christiano, L., M. Eichenbaum, And C. Evans (2005): "Nominal Rigidities and the Dynamic Effects of a Shock to Monetary Policy," Journal of Political Economy, 113.

Dossche, M., F. Heylen, And D. Van den Poel (2010): "The kinked demand curve and price rigidity: Evidence from scanner data," Scandinavian Journal of Economics, 112, $723-752$.

Dow, J. And S. Werlang (1992): "Uncertainty Aversion, Risk Aversion, and the Optimal Choice of Portfolio," Econometrica, 60, 197-204.

Dupraz, S. (2016): "A Kinked-Demand Theory of Price Rigidity," Mimeo, Banque de France. 
Eichenbaum, M., N. Jaimovich, and S. Rebelo (2011): "Reference Prices, Costs, and Nominal Rigidities," American Economic Review, 101, 234-62.

Einhorn, H. J. AND R. M. Hogarth (1986): "Decision making under ambiguity," Journal of Business, S225-S250.

EllsberG, D. (1961): "Risk, Ambiguity, and the Savage Axioms," The Quarterly Journal of Economics, 643-669.

Epstein, L. G. And M. Schneider (2003): "Recursive Multiple-Priors," Journal of Economic Theory, 113, 1-31.

Gagnon, E., D. López-SAlido, And N. Vincent (2012): "Individual Price Adjustment along the Extensive Margin," NBER Macroeconomics Annual, 27, 235-281.

Gaur, V., S. Kesavan, A. Raman, And M. L. Fisher (2007): "Estimating demand uncertainty using judgmental forecasts," Manufacturing 83 Service Operations Management, 9, 480-491.

Gilboa, I. And D. Schmeidler (1989): "Maxmin Expected Utility with Non-unique Prior," Journal of Mathematical Economics, 18, 141-153.

Golosov, M. And R. E. LuCas (2007): "Menu costs and phillips curves," Journal of Political Economy, 115, 171-199.

Handel, B. R. And K. Misra (2015): "Robust new product pricing," Marketing Science, $34,864-881$.

JordÁ, O. (2005): "Estimation and Inference of Impulse Responses by Local Projections," American Economic Review, 95, 161-182.

Kehoe, P. And V. Midrigan (2015): "Prices are sticky after all," Journal of Monetary Economics, 75, 35-53.

Kimball, M. S. (1995): "The Quantitative Analytics of the Basic Neomonetarist Model," Journal of Money, Credit, and Banking, 27.

Klenow, P. J. And O. Kryvtsov (2008): "State-Dependent or Time-Dependent Pricing: Does It Matter for Recent US Inflation?" The Quarterly Journal of Economics, 863-904.

Klenow, P. J. And B. A. Malin (2010): "Microeconomic Evidence on Price-Setting," Handbook of Monetary Economics, 3, 231-284.

Klenow, P. J. AND J. L. Willis (2016): "Real rigidities and nominal price changes," Economica, 83, 443-472.

Knotek, I. And S. Edward (2010): "A Tale of Two Rigidities: Sticky Prices in a StickyInformation Environment," Journal of Money, Credit and Banking, 42, 1543-1564. 
Kunreuther, H., R. Hogarth, and J. Meszaros (1993): "Insurer ambiguity and market failure," Journal of Risk and Uncertainty, 7, 71-87.

Machina, M. J. And M. Siniscalchi (2014): "Ambiguity and ambiguity aversion," in Handbook of the Economics of Risk and Uncertainty, Elsevier, vol. 1, 729-807.

Mackowiak, B. And M. Wiederholt (2009): "Optimal Sticky Prices under Rational Inattention," American Economic Review, 99, 769-803.

Maffioletti, A. And M. Santoni (2005): "Do trade union leaders violate subjective expected utility? Some insights from experimental data," Theory and Decision, 59, 207253.

Mankiw, N. G. AND R. ReIs (2002): "Sticky Information versus Sticky Prices: A Proposal to Replace the New Keynesian Phillips Curve," The Quarterly Journal of Economics, 117, $1295-1328$.

MARCh, J. G. AND Z. ShaPIRA (1987): "Managerial perspectives on risk and risk taking," Management science, 33, 1404-1418.

MatĚJKA, F. (2015): "Rationally inattentive seller: Sales and discrete pricing," The Review of Economic Studies, 83, 1125-1155.

Nakamura, E. And J. Steinsson (2008): "Five facts about prices: A reevaluation of menu cost models," The Quarterly Journal of Economics, 123, 1415-1464.

Raman, A., M. Fisher, And A. McClelland (2001): Supply chain management at World Co., Ltd, Harvard Business School Boston, MA.

Rasmussen, C. E. And C. K. Williams (2006): Gaussian processes for machine learning, vol. 1, MIT press Cambridge.

ReIs, R. (2006): "Inattentive producers," The Review of Economic Studies, 73, 793-821.

Rothschild, M. (1974): "A two-armed bandit theory of market pricing," Journal of Economic Theory, 9, 185-202.

Sims, C. A. (2003): "Implications of rational inattention," Journal of monetary Economics, $50,665-690$.

Stevens, L. (2014): "Coarse Pricing Policies," Manuscript, Univ. of Maryland.

Stigler, G. J. (1947): "The kinky oligopoly demand curve and rigid prices," The Journal of Political Economy, 432-449.

Stiglitz, J. E. (1979): "Equilibrium in product markets with imperfect information," The American Economic Review, 339-345.

VAvRA, J. (2014): "Inflation Dynamics and Time-Varying Volatility: New Evidence and an Ss Interpretation," The Quarterly Journal of Economics, 129, 215-258. 
Woodford, M. (2003): "Imperfect Common Knowledge and the Effects of Monetary Policy," Knowledge, Information, and Expectations in Modern Macroeconomics: In Honor of Edmund S. Phelps, 25.

(2009): "Information-constrained state-dependent pricing," Journal of Monetary Economics, 56, S100-S124. 


\section{A Online Appendix A}

\section{A.1 Updating with more observed prices}

We can readily expand the updating formulas that we have developed in Section 2.2 for one observed price to the case of multiple observed past price points. Let the firm's information set $\varepsilon^{t-1}$ contain $T$ unique price points collected in the vector $\mathbf{p}_{T}=\left[p_{1}, \ldots, p_{T}\right]^{\prime}$, where $T>0$ is arbitrarily large but finite. We label the average realized quantity sold at each of these unique price points $\bar{y}_{i}$, and similarly collect them in the vector $\mathbf{y}_{T}=\left[y_{1}, \ldots, y_{T}\right]^{\prime}$. Lastly, let $N_{i}$ be the number of times the firm has seen price point $p_{i}$ in the past, and thus this is the number of signals at $p_{i}$ the firm has. The vector $\mathbf{N}_{T}=\left[N_{1}, \ldots, N_{T}\right]^{\prime}$ collects these values.

The joint distribution between demand at any price $p$ and the vector of signals $\mathbf{y}$ is similarly joint Normal:

$$
\left[\begin{array}{c}
x(p) \\
\mathbf{y}_{T}
\end{array}\right] \sim N\left(\left[\begin{array}{c}
m(p) \\
m\left(\mathbf{p}_{T}\right)
\end{array}\right], \Sigma\left(p, \mathbf{p}_{T}\right)\right)
$$

where the variance-covariance matrix is given by

$$
\Sigma\left(p, \mathbf{p}_{T}\right)=\left[\begin{array}{cc}
\sigma_{x}^{2} & K\left(p, \mathbf{p}_{T}\right) \\
K\left(\mathbf{p}_{T}, p\right) & K\left(\mathbf{p}_{T}, \mathbf{p}_{T}\right)+\operatorname{diag}\left(\mathbf{N}_{T}\right)^{-1} \sigma_{z}^{2}
\end{array}\right]
$$

The conditional expectation of $x(p)$ given a prior mean function $m(p)$ and the vector of signals $\mathbf{y}_{T}$, follows from applying the standard formula for conditional Gaussian expectations:

$$
E\left(x(p) \mid \mathbf{y}_{T}, m(p)\right)=m(p)+K\left(p, \mathbf{p}_{T}\right)\left(K\left(\mathbf{p}_{T}, \mathbf{p}_{T}\right)+\operatorname{diag}\left(\mathbf{N}_{T}\right)^{-1} \sigma_{z}^{2}\right)^{-1}\left(\mathbf{y}_{T}-m\left(\mathbf{p}_{T}\right)\right)
$$

Expanding the above expression, we can show that the conditional expectation is again linear in the prior and a weighted sum of the demeaned signals, leading to

$$
E\left(x(p) \mid \mathbf{y}_{T}, m(p)\right)=m(p)+\alpha_{1}(p)\left(y_{1}-m\left(p_{1}\right)\right)+\cdots+\alpha_{T}(p)\left(y_{T}-m\left(p_{T}\right)\right)
$$

where $\alpha_{i} \in(0,1)$ is the $i$-th element of the $1 \mathrm{x} T$ vector $K\left(p, \mathbf{p}_{T}\right)\left(K\left(\mathbf{p}_{T}, \mathbf{p}_{T}\right)+\operatorname{diag}\left(\mathbf{N}_{T}\right)^{-1} \sigma_{z}^{2}\right)^{-1}$.

Without loss of generality, assume the prices in $\mathbf{p}$ are sorted in ascending order, with the last element being the largest price value. In building the worst case expectation, one can work from back to front and first characterize the worst case prior $m^{*}\left(p ; p_{t}\right)$ for entertained price values $p_{t}>p_{T}$. The firm wants the prior level of demand at the entertained price $p_{t}, m^{*}\left(p_{t} ; p_{t}\right)$, to be the lowest possible so it sets it equal to the lower bound of $\Upsilon_{0}$ so that

$$
m^{*}\left(p_{t} ; p_{t}\right)=-\gamma-b p_{t}
$$

Again similar to the case of only one previously observed price, the firm is worried that demand 
decreases a lot as it increases its price away from its previous observations. Now, however, this worry does not apply only to the closest signal at the price value of $p_{T}$, but to all previous signals. Since all previous signals were observed at prices below $p_{t}$, the worst case $m^{*}\left(p ; p_{t}\right)$ for any $p<p_{t}$ is given by:

$$
m^{*}\left(p ; p_{t}\right)=\min \left[\gamma-b p,-\gamma-b p_{t}+(b+\delta)\left(p_{t}-p\right)\right]
$$

Next consider, $p_{t} \in\left(p_{T-1}, p_{T}\right]$. The worst case $m^{*}\left(p_{t} ; p_{t}\right)$ is again at the lower bound of the admissible set $\Upsilon_{0}$. And the basic intuition for the rest of the worst-case prior is similar to before - the firm worries that setting the price $p_{t}$ away from its previous observations $\mathbf{p}_{T}$ makes demand change for the worse. Thus, the firm is worried that $m^{*}\left(\mathbf{p}_{T} ; p_{t}\right)$ is the highest possible level, given constraints on the admissible set $\Upsilon_{0}$ and the fact that $m^{*}\left(p_{t} ; p_{t}\right)=-\gamma-b p_{t}$. This concern yields

$$
m^{*}\left(p ; p_{t}\right)= \begin{cases}\min \left[\gamma-b p,-\gamma-b p_{t}+(b+\delta)\left(p_{t}-p\right)\right] & \text { for } p<p_{t} \\ \min \left[\gamma-b p,-\gamma-b p_{t}+(b-\delta)\left(p_{t}-p\right)\right] & \text { for } p \geq p_{t}\end{cases}
$$

Hence for all price points below the currently entertained price $p_{t}$, the worst-case prior is restricted by the maximum admissible derivative $b+\delta$, while for prices above $p_{t}$ it is restricted by the lowest admissible derivative $b-\delta$.

Substituting this worst case prior in (34), it is easy to evaluate the worst-case expectation $\widehat{x}^{*}\left(p_{t} \mid \mathbf{y}_{T}, m^{*}\left(p ; p_{t}\right)\right)$. Given the piecewise nature of $m^{*}\left(p ; p_{t}\right)$, it follows that there is a kink in the worst-case expected demand $\widehat{x}^{*}\left(p_{t} \mid \mathbf{y}_{T}, m^{*}\left(p ; p_{t}\right)\right)$ around any $p \in \mathbf{p}_{T}$.

\section{A.2 Proofs for Section 2}

Proposition 1. Define $\delta^{*}=\delta \operatorname{sgn}\left(p_{t}-p_{0}\right)$. For a given realization of $c_{t}$, the difference in worstcase expected profits at $p_{t}$ and $p_{0}$, up to a first-order approximation around $p_{0}$, is

$$
\ln v^{*}\left(\varepsilon^{t-1}, c_{t}, p_{t}\right)-\ln v_{0}^{*}\left(\varepsilon^{t-1}, c_{t}, p_{0}\right) \approx\left[\frac{e^{p_{0}}}{e^{p_{0}}-e^{c_{t}}}-\left(b+\alpha_{t-1}\left(p_{0}\right) \delta^{*}\right)\right]\left(p_{t}-p_{0}\right)
$$

Proof. Consider $\ln v^{*}\left(\varepsilon^{t-1}, c_{t}, p_{t}\right)$ at some $p_{t} \in\left[p_{0}-\frac{2 \gamma}{\delta}, p_{0}+\frac{2 \gamma}{\delta}\right]$. When $p_{t}>p_{0}$, we have

$$
\ln \left(e^{p_{t}}-e^{c_{t}}\right)+\left\{-\gamma-b p_{t}+\alpha_{t-1}\left(p_{t}\right) \widehat{z}_{0}-\alpha_{t-1}\left(p_{t}\right) \delta\left(p_{t}-p_{0}\right)+.5 \widehat{\sigma}_{t-1}^{2}\left(p_{t}\right)+.5 \sigma_{z}^{2}\right\}
$$

while at $p_{t}<p_{0}$, this equals

$$
\ln \left(e^{p_{t}}-e^{c_{t}}\right)+\left\{-\gamma-b p_{t}+\alpha_{t-1}\left(p_{t}\right) \widehat{z}_{0}+\alpha_{t-1}\left(p_{t}\right) \delta\left(p_{t}-p_{0}\right)+.5 \widehat{\sigma}_{t-1}^{2}\left(p_{t}\right)+.5 \sigma_{z}^{2}\right\}
$$

where for convenience we have defined $\widehat{z}_{0} \equiv-\gamma-b p_{0}$. In turn, $\ln v^{*}\left(\varepsilon^{t-1}, c_{t}, p_{0}\right)$ equals

$$
\ln \left(e^{p_{t}}-e^{c_{t}}\right)+\left\{-\gamma-b p_{0}+\alpha_{t-1}\left(p_{t}\right) \widehat{z}_{0}+.5 \widehat{\sigma}_{t-1}^{2}\left(p_{0}\right)+.5 \sigma_{z}^{2}\right\}
$$


Fix some $c_{t}$ and take a first-order approximation of $\ln v^{*}\left(\varepsilon^{t-1}, c_{t}, p_{t}\right)$ with respect to $p_{t}$, evaluated at $p_{0}$. Since this function is not differentiable at $p_{0}$, we analyze its right and left derivative separately. The former derivative equals

$$
\frac{e^{p_{0}}}{e^{p_{0}}-e^{c_{t}}}-b-\alpha_{t-1}\left(p_{0}\right) \delta+\frac{\partial \alpha_{t-1}\left(p_{t}\right)}{\partial p_{t}}\left[\widehat{z}_{0}-\delta\left(p_{t}-p_{0}\right)\right]+.5 \frac{\partial \widehat{\sigma}_{t-1}^{2}\left(p_{t}\right)}{\partial p_{t}}
$$

where the partial derivatives $\frac{\partial \alpha_{t-1}\left(p_{t}\right)}{\partial p_{t}}$ and $\frac{\partial \widehat{\sigma}_{t-1}^{2}\left(p_{t}\right)}{\partial p_{t}}$ are evaluated locally at $p_{0}$. In particular, given that

$$
\alpha_{t-1}\left(p_{t}\right)=\frac{\sigma_{x}^{2}}{\sigma_{x}^{2}+\sigma_{z}^{2} / N_{0}} e^{-\psi\left(p_{t}-p_{0}\right)^{2}} ; \widehat{\sigma}_{t-1}^{2}\left(p_{t}\right)=\sigma_{x}^{2}\left(1-\alpha_{t-1}\left(p_{t}\right)\right),
$$

then these two functions are differentiable $p_{0}$, with marginal effects equal to zero at $p_{0}$. Therefore, the local approximation to the right of $p_{0}$ simplifies to

$$
\frac{e^{p_{0}}}{e^{p_{0}}-e^{c_{t}}}-\left[b+\alpha_{t-1}\left(p_{0}\right) \delta\right]
$$

The first term in the brackets reflects the effect of changing the price on profits, while the second captures the movement of demand along a curve with elasticity $-b$. The third term arises from the effect of demand of moving along a steeper demand curve, which is a characteristic of the worst-case belief about the demand elasticity.

Therefore, we obtain the local approximation to the right of $p_{0}$

$$
\ln v^{*}\left(\varepsilon^{t-1}, c_{t}, p_{t}\right)-\ln v_{0}^{*}\left(\varepsilon^{t-1}, c_{t}, p_{0}\right) \approx\left[\frac{e^{p_{0}}}{e^{p_{0}}-e^{c_{t}}}-\left(b+\alpha_{t-1}\left(p_{0}\right) \delta\right)\right]\left(p_{t}-p_{0}\right)
$$

A similar derivation follows for the derivative to the left of $p_{0}$, where we obtain

$$
\frac{e^{p_{0}}}{e^{p_{0}}-e^{c_{t}}}-\left[b-\alpha_{t-1}\left(p_{0}\right) \delta\right]
$$

and therefore the local approximation to the left of $p_{0}$ is simply

$$
\ln v^{*}\left(\varepsilon^{t-1}, c_{t}, p_{t}\right)-\ln v_{0}^{*}\left(\varepsilon^{t-1}, c_{t}, p_{0}\right) \approx\left[\frac{e^{p_{0}}}{e^{p_{0}}-e^{c_{t}}}-\left(b-\alpha_{t-1}\left(p_{0}\right) \delta\right)\right]\left(p_{t}-p_{0}\right)
$$

We obtain the result in Proposition 1 by putting together equations (35) and (36) and using the signum function to define $\delta^{*}=\delta \operatorname{sgn}\left(p_{t}-p_{0}\right)$.

Proposition 2. Let $\delta_{i}^{*} \equiv \delta \operatorname{sgn}\left(p_{t}-p_{i}\right)$ for all $p_{i} \in \varepsilon^{t-1}$. For a given realization of $c_{t}$, up to a first-order approximation around each such $p_{i} \in \varepsilon^{t-1}$ :

$$
\ln v^{*}\left(\varepsilon^{t-1}, c_{t}, p_{t}\right)-\ln v_{0}^{*}\left(\varepsilon^{t-1}, c_{t}, p_{i}\right) \approx\left[\frac{e^{p_{i}}}{e^{p_{i}}-e^{c_{t}}}-\left(b+\alpha_{t-1, i}\left(p_{i}\right) \delta^{*}+A_{i}\right)\right]\left(p_{t}-p_{i}\right) .
$$


Proof. The structure of the proof is very similar to the previous one. Consider $\ln v^{*}\left(\varepsilon^{t-1}, c_{t}, p_{t}\right)$ at some $p_{t} \in\left[p_{i}-\frac{2 \gamma}{\delta}, p_{i}+\frac{2 \gamma}{\delta}\right]$. Using $\delta_{i}^{*} \equiv \delta \operatorname{sgn}\left(p_{t}-p_{i}\right)$ we can write $\ln v^{*}\left(\varepsilon^{t-1}, c_{t}, p_{t}\right)$ as

$\ln \left(e^{p_{t}}-e^{c_{t}}\right)+\left\{-\gamma-b p_{t}+\sum_{p_{k} \in \varepsilon^{t-1}} \alpha_{t-1, k}\left(p_{t}\right)\left(\widehat{z}_{k}-\delta_{k}^{*}\left(p_{t}-p_{k}\right) \mathbb{1}\left(p_{t} \in\left(\underline{p}_{k}, \bar{p}_{k}\right)\right)\right)+.5 \widehat{\sigma}_{t-1}^{2}\left(p_{t}\right)+.5 \sigma_{z}^{2}\right\}$,

Fixing some $c_{t}$, take a first-order approximation of $\ln v^{*}\left(\varepsilon^{t-1}, c_{t}, p_{t}\right)$ with respect to $p_{t}$, evaluated at $p_{i}$. Since this function is not differentiable at $p_{0}$, we analyze its right and left derivative separately as before, Using the notation $\delta_{i}^{*} \equiv \delta \operatorname{sgn}\left(p_{t}-p_{i}\right)$, we can express both the right and left derivatives around one of the $p_{i} \in \varepsilon^{t-1}$ as

$$
\begin{aligned}
\frac{e^{p_{i}}}{e^{p_{i}}-e^{c_{t}}}-b & -\alpha_{t-1, i}\left(p_{i}\right) \delta_{i}^{*}+\frac{\partial \alpha_{t-1, i}\left(p_{i}\right)}{\partial p} \widehat{z}_{i}+.5 \frac{\partial \widehat{\sigma}_{t-1}^{2}\left(p_{i}\right)}{\partial p} \\
& +\sum_{p_{k} \in \varepsilon^{t-1} / p_{i}} \frac{\partial \alpha_{t-1, k}\left(p_{i}\right)}{\partial p}\left(\widehat{z}_{k}-\delta_{k}^{*}\left(p_{i}-p_{k}\right) \mathbb{1}\left(p_{i} \in\left(\underline{p}_{k}, \bar{p}_{k}\right)\right)\right)-\sum_{p_{k} \in \varepsilon^{t-1} / p_{i}} \alpha_{t-1, k}\left(p_{i}\right)\left(-\delta_{k}^{*} \mathbb{1}\left(p_{i} \in\left(\underline{p}_{k}, \bar{p}_{k}\right)\right)\right)
\end{aligned}
$$

The partial derivatives of the signal-to-noise ratios and the posterior variance are no longer zero, however they are not a function of the sign of $\left(p_{t}-p_{i}\right)$ hence when considering a local approximation around $p_{i}$ all of the additional terms (as compared to Proposition 1) can be treated as a constant. We call that constant $A_{i}$ :

$$
\begin{aligned}
A_{i} & =\frac{\partial \alpha_{t-1, i}\left(p_{i}\right)}{\partial p} \widehat{z}_{i}+.5 \frac{\partial \widehat{\sigma}_{t-1}^{2}\left(p_{i}\right)}{\partial p} \\
& +\sum_{p_{k} \in \varepsilon^{t-1} / p_{i}} \frac{\partial \alpha_{t-1, k}\left(p_{i}\right)}{\partial p}\left(\widehat{z}_{k}-\delta_{k}^{*}\left(p_{i}-p_{k}\right) \mathbb{1}\left(p_{i} \in\left(\underline{p}_{k}, \bar{p}_{k}\right)\right)\right)-\sum_{p_{k} \in \varepsilon^{t-1} / p_{i}} \alpha_{t-1, k}\left(p_{i}\right)\left(-\delta_{k}^{*} \mathbb{1}\left(p_{i} \in\left(\underline{p}_{k}, \bar{p}_{k}\right)\right)\right)
\end{aligned}
$$

Using the fact that the $A_{i}$ term is not a function of $p_{t}$, it just updates the coefficients in the first-order approximation of $\ln v^{*}\left(\varepsilon^{t-1}, c_{t}, p_{t}\right)$, but does not change the basic observation that there is a kink in the profit function at $p_{i}$, so that:

$$
\ln v^{*}\left(\varepsilon^{t-1}, c_{t}, p_{t}\right)-\ln v_{0}^{*}\left(\varepsilon^{t-1}, c_{t}, p_{i}\right) \approx\left[\frac{e^{p_{i}}}{e^{p_{i}}-e^{c_{t}}}-\left(b+\alpha_{t-1, i}\left(p_{i}\right) \delta^{*}+A_{i}\right)\right]\left(p_{t}-p_{i}\right)
$$

\section{A.3 Forward looking behavior}

We solve the recursive optimization problem in two steps. First, we compute the value function at time $t+1$. The key insight is that from this point onward the firm solves a series of static maximization problems because the endogenous state variable, the information set $\varepsilon^{t}$, remains the 
same from period to period. Still, the firm faces a dynamic, recursive problem because of the law of motion of the exogenous state variable, the cost shock $c_{t}$, which evolves according to its law of motion $g\left(c_{t+1} \mid c_{t}\right)$. Hence, the value function at $t+1$, which we label with $\tilde{V}($.) to differentiate from the time- $t$ value function $V($.$) , is given by$

$$
\tilde{V}\left(\varepsilon^{t}, c_{t+1}\right)=\max _{p_{t+1}} \min _{m(p) \in \Upsilon_{0}} E\left[\nu\left(\varepsilon_{t+1}, c_{t+1}\right)+\beta \int \tilde{V}\left(\varepsilon^{t}, c_{t+2}\right) g\left(c_{t+2} \mid c_{t+1}\right) d c_{t+2} \mid \varepsilon^{t}\right]
$$

Since the information set is not growing over time, the state space for this problem is finite and tractable. As a result, we can solve for $\tilde{V}\left(\varepsilon^{t}, c_{t+1}\right)$ through standard techniques and use it as the continuation value perceived by the firm at time $t$ :

$$
V\left(\varepsilon^{t-1}, c_{t}\right)=\max _{p_{t}} \min _{m(p) \in \Upsilon_{0}} E\left[\nu\left(\varepsilon_{t}, c_{t}\right)+\beta \int \tilde{V}\left(\varepsilon^{t}, c_{t+1}\right) g\left(c_{t+1} \mid c_{t}\right) d c_{t+1} \mid \varepsilon^{t-1}\right]
$$

s.t.

$$
\varepsilon^{t}=\left\{\varepsilon^{t-1}, p_{t}, y_{t}\right\}
$$

Thus, at time $t$ the firm fully takes into account that $p_{t}$, and the resulting new demand signal $y_{t}$, will serve as informative signals for future profit-maximization decisions. Importantly, this information is useful not only in the very next period, but propagates through the infinite future according to the law of motion of $c_{t}$.

For the following analytical results we work with the case where $\psi=\infty$ and the firm has perfect foresight on future costs, s.t. $c_{t+k}=c$ for all $k \geq 1$, for some constant $c$. In this case, the time $t+1$ value function is just the present discounted value of worst-case expected profits when the cost shock equals $c$ :

$$
\tilde{V}\left(\varepsilon^{t}, c\right)=\frac{\max _{p} \min _{m(p) \in \Upsilon_{0}} E\left[\nu\left(\varepsilon_{t+1}, c\right) \mid \varepsilon^{t}\right]}{1-\beta}
$$

Hence, the only remaining uncertainty in $\tilde{V}($.$) from the perspective of time t$ is the uncertainty about the realization of the time $t$ signal $y_{t}$. Next, we turn to characterizing the expectation of $\tilde{V}$, given the time $\mathrm{t}$ information set $\varepsilon^{t-1}$.

For all analytical results below, we assume that (i) $\psi \rightarrow \infty$ and (ii) there is perfect foresight on future costs so that $c_{t+k}=c$ for some $\mathrm{c}$.

\section{Exploration makes prices more flexible when $\varepsilon^{t-1}$ contains demand observations at} only one previous price $p_{0}$

We start with the case where the time $t$ information set, $\varepsilon^{t-1}$, contains only one price point, $p_{0}$, observed $N_{0}$ times with an average signal $y_{0}$. To be specific, call that information set $\varepsilon^{0}$. We will assume that the realization of the signal $y_{0}$ is good enough, so that when $c=c_{0}^{*}=p_{0}-\ln \left(\frac{b}{b-1}\right)$, 
$p_{0}$ is not just locally optimal (recall Corollary 1 ), but that it is the global maximizer conditional on $\varepsilon^{t-1}$. The relevant condition is

$$
\widehat{z}_{0}=y_{0}-\left(-\gamma-b p_{0}\right)>\frac{\sigma_{x}^{2}}{2}
$$

in which case

$$
p_{0}=\arg \max _{p} \min _{m(p) \in \Upsilon_{0}} E\left[\nu\left(\varepsilon_{t+1}, c_{0}^{*}\right) \mid \varepsilon^{0}, m(p)\right]
$$

Hence in the absence of any new information, in future periods the firm will optimally set $p_{0}$, since it essentially faces a static problem with marginal cost equal to $c_{0}^{*}$. The signal pair $\left\{p_{t}, y_{t}\right\}$ provides such new information and could lead to a different optimal action $p_{t+k}$.

Our first result is a characterization of the current price $p_{t}$ that maximizes the expected continuation value when $c=c_{0}^{*}$. It turns out that when the firm has collected prior information about demand only at $p_{0}$, then even even at that value of the cost the optimal exploration strategy is to deviate from $p_{0}$.

Proposition 3. The expected continuation value $E\left[\tilde{V}\left(\left\{\varepsilon^{0}, p_{t}, y_{t}\right\}, c_{0}^{*}\right) \mid \varepsilon^{0}, p_{t}\right]$ achieves its maximum at

$$
p_{t}^{*}=\arg \min _{p}\left(p-p_{0}\right)^{2} \text { s.t. } p \neq p_{0}
$$

Proof. In order to simplify notation, throughout the proofs we will use the standard expectation notation $E($.$) to define the worst-case expectation of the firm.$

The limiting case $\psi \rightarrow \infty$ simplifies the construction of the worst-case expected demand because $\operatorname{corr}\left(x(p), x\left(p^{\prime}\right)\right)=0$ for all $p \neq p^{\prime}$. Thus, when updating beliefs about demand at any price $p$, only past signals observed at that particular price $p$ matter. For future reference, it will be convenient to define the following notation for signal-to-noise ratios that will show up repeatedly

$$
\begin{gathered}
\alpha_{0} \equiv \alpha_{t-1}\left(p_{0} ; p_{0}\right)=\frac{\sigma_{x}^{2}}{\sigma_{x}^{2}+\sigma_{z}^{2} / N_{0}} \\
\alpha_{t \mid 0} \equiv \alpha_{t}\left(p_{0} ; p_{0} \mid p_{t}=p_{0}\right)=\frac{\sigma_{x}^{2}}{\sigma_{x}^{2}\left(N_{0}+1\right)+\sigma_{z}^{2}} \\
\alpha_{t} \equiv \alpha_{t-1}\left(p_{t} ; p_{t} \mid p_{t} \neq p_{0}\right)=\frac{\sigma_{x}^{2}}{\sigma_{x}^{2}+\sigma_{z}^{2}}
\end{gathered}
$$

where the first is the signal-to-noise ratio of the signal $y_{0}$ conditional on $\varepsilon^{0}$ information, $\alpha_{t \mid 0}$ and $\alpha_{t}$ are the (recursive) signal-to-noise ratios applicable to the new signal $y_{t}$ given the signal $y_{0}$, in the two cases where $p_{t}=p_{0}$ and $p_{t} \neq p_{0}$ respectively. Since $p_{0}=\ln \left(\frac{b}{b-1}\right)+c_{0}^{*}$, it is the optimal myopic price for $c_{t+k}=c_{0}^{*}$, which is the relevant case in the future. Thus, if its information set does not change, the firm will price $p_{t+k}=p_{0}$ in the future. The information set changes, of course, as a function of the current period pricing choice $p_{t}$ and the resulting new signal $y_{t}$. For convenience, 
define the perceived innovations in the existing signal $y_{0}$ and the new signal $y_{t}$ as

$$
\begin{gathered}
\widehat{z}_{0} \equiv y_{0}-\left(-\gamma-b p_{0}\right) \\
\widehat{z}_{t} \equiv y_{t}-\left(-\gamma-b p_{t}\right)
\end{gathered}
$$

and the variance adjusted innovation of $y_{0}$ as

$$
\widetilde{z}_{0} \equiv \widehat{z}_{0}-\frac{1}{2} \sigma_{x}^{2}
$$

Observe that since $c_{t+k}=c_{0}^{*}$ with probability one, the only uncertainty over future profits is in the innovation of the new signal $\widehat{z}_{t}$. Hence, the expected continuation value is simply the expected discounted value of a stream of worst-case static profits at $c_{t+k}=c_{0}^{*}$, after taking the expectation over the unknown $\widehat{z}_{t}: E\left[\tilde{V}\left(\left\{\varepsilon^{0}, p_{t}, y_{t}\right\}, c_{0}^{*}\right) \mid \varepsilon^{0}, p_{t}\right]=\frac{\beta}{1-\beta} E\left[E\left(\nu\left(p_{t+k}^{*}, c_{0}^{*}\right) \mid\left\{\varepsilon^{0}, p_{t}, y_{t}\right\}\right) \mid \varepsilon^{0}, p_{t}\right]=$ $\frac{\beta}{1-\beta} E\left[\nu_{t+k}^{*}\left(p_{t+k}^{*}, c_{0}^{*}\right) \mid \varepsilon^{0}, p_{t}\right]$, where $p_{t+k}^{*}$ is the resulting static optimal price, given the updated information set $\left\{\varepsilon^{0}, p_{t}, y_{t}\right\}$.

If $p_{t}=p_{0}$, this optimal price is still $p_{0}$ unless the information in the new signal $y_{t}$ is particularly bad and sufficiently erodes the firm's beliefs about profits at $p_{0}$, in which case the firm switches to the interior optimal price $p_{t+k}^{i n t}$ - the ex-ante second best option. To find this interior optimum, note that for all prices $p_{t+k} \neq p_{0}$ the worst-case demand is simply

$$
\widehat{x}_{t}^{*}\left(p_{t+k} ; m^{*}\left(p ; p_{t+k}\right)\right)=-\gamma-b p
$$

hence the interior optimal price is

$$
p_{t+k}^{\text {int }}=\min \left\{p \mid\left(p-p_{0}\right)^{2}>0\right\}
$$

which gets you as close as possible the to optimal markup $\frac{b}{b-1}$ while still staying on the smooth portion of the firm's demand curve (recall: there is a kink in the worst-case belief at $p_{0}$, but is smooth everywhere else). Thus, if $p_{t}=p_{0}$, optimal $p_{t+k}^{*}$ is equal to $p_{0}$ unless $\widehat{z}_{t}<\underline{z}_{0}$, where $\underline{z}_{0}$ is such that:

$$
\frac{E_{t-1}\left(\nu_{t+k}^{*}\left(p_{0}, c_{0}^{*}\right) \mid \varepsilon^{0}, p_{t}=p_{0}, \widehat{z}_{t}=\underline{\mathrm{z}}_{0}\right)}{\lim _{p \rightarrow p_{0}} E\left(\nu_{t+k}^{*}\left(p, c_{0}^{*}\right) \mid \varepsilon^{0}, p_{t}=p_{0}, \widehat{z}_{t}=\underline{\mathrm{z}}_{0}\right)}=1
$$

Substituting in the relevant expressions and simplifying, we can derive

$$
\underline{\mathrm{z}}_{0}=\frac{\sigma_{x}^{2}}{2}\left(1-\alpha_{0}\right)-\frac{\alpha\left(p_{0}\right)}{\alpha_{t \mid 0}} \widetilde{z}_{0} .
$$

Hence if $p_{t}=p_{0}$, the optimal $p_{t+k}^{*}$ is equal to $p_{0}$ as long as the innovation in the new signal is good enough - namely $\widehat{z}_{t} \geq \underline{z}_{0}$. 
If $p_{t} \neq p_{0}, p_{0}$ remains the optimal price at $t+k$ unless the new signal $y_{t}$ is good enough to convince the firm to deviate from its ex-ante optimum $p_{0}$ and move to the newly observed $p_{t}$ itself. In the limiting case $\psi \rightarrow \infty$ we know that the only potential alternative is $p_{t}$, because $y_{t}$ does not update beliefs anywhere else, and hence $p_{0}$ dominates all other prices. In particular, for every possible $p_{t}$ there is an upper threshold for the innovation in $y_{t}$, such that $p_{t+k}^{*}=p_{t}$ if and only if $\widehat{z}_{t}>\bar{z}\left(p_{t}\right)$. This threshold $\bar{z}\left(p_{t}\right)$ satisfies

$$
\frac{E\left(\nu_{t+k}^{*}\left(p_{t}, c_{0}^{*}\right) \mid \varepsilon^{0}, p_{t} \neq p_{0}, \widehat{z}_{t}=\bar{z}\left(p_{t}\right)\right)}{E\left(\nu_{t+k}^{*}\left(p_{0}, c_{0}^{*}\right) \mid \varepsilon^{0}, p_{t} \neq p_{0}, \widehat{z}_{t}=\bar{z}\left(p_{t}\right)\right)}=1
$$

Substituting in the respective expressions, and simplifying we can derive:

$$
\bar{z}\left(p_{t}\right)=\frac{\alpha_{0}}{\alpha_{t}} \widetilde{z}_{0}+\frac{\sigma_{x}^{2}}{2}-\frac{1}{\alpha_{t}}\left[\ln \left(\frac{\exp \left(p_{t}\right)-\exp \left(c_{0}^{*}\right)}{\exp \left(p_{0}\right)-\exp \left(c_{0}^{*}\right)}\right)+b\left(p_{0}-p_{t}\right)\right]
$$

With the two thresholds thusly characterized, we can conclude that the optimal pricing policy at time $t+k$ is given by:

$$
p_{t+k}^{*}= \begin{cases}p_{0} & \text { if } p_{t}=p_{0} \text { and } \widehat{z}_{t} \geq \underline{z}_{0} \text { or } p_{t} \neq p_{0} \text { and } \widehat{z}\left(p_{t}\right) \leq \bar{z}\left(p_{t}\right) \\ p_{t} & \text { if } p_{t} \neq p_{0} \text { and } \widehat{z}_{t}>\bar{z}\left(p_{t}\right) \\ p_{t+k}^{\text {int }} & \text { if } p_{t}=p_{0} \text { and } \widehat{z}_{t}<\underline{z}_{0}\end{cases}
$$

We can then evaluate the expected continuation value $E\left[\tilde{V}\left(\left\{\varepsilon^{0}, p_{t}, y_{t}\right\}, c_{0}^{*}\right) \mid \varepsilon^{0}, p_{t}\right]$ - we do so separately for the cases $p_{t}=p_{0}$ and $p_{t} \neq p_{0}$, since the expected continuation value (which we will denote by the short-hand $E_{t-1}(\tilde{V})$ to save space) is potentially discontinuous at $p_{t}=p_{0}$, so that $E_{t-1}\left(\tilde{V} \mid p_{t}=p_{0}\right)=$

$$
\begin{aligned}
& =\Phi\left(\frac{\underline{\mathrm{z}}_{0}}{\sqrt{\sigma_{x}^{2}\left(1-\alpha_{0}\right)+\sigma_{z}^{2}}}\right)\left(\exp \left(p_{0}\right)-\exp \left(c_{0}^{*}\right)\right) \exp \left(-\gamma-b p_{0}+\frac{1}{2}\left(\sigma_{x}^{2}+\sigma_{z}^{2}\right)\right) \\
& +\left(1-\Phi\left(\frac{\underline{\mathrm{z}}_{0}}{\sqrt{\sigma_{x}^{2}\left(1-\alpha_{0}\right)+\sigma_{z}^{2}}}\right)\right)\left(\exp \left(p_{0}\right)-\exp \left(c_{0}^{*}\right)\right) \exp \left(-\gamma-b p_{0}+\alpha_{0} \widehat{z}_{0}+\frac{1}{2}\left(\sigma_{x}^{2}\left(1-\alpha_{0}\right)+\sigma_{z}^{2}\right)\right) \frac{\Phi\left(\frac{\alpha_{t \mid 0}\left(\sigma_{x}^{2}\left(1-\alpha_{0}\right)+\sigma_{z}^{2}\right)-\underline{\mathrm{z}}_{0}}{\sqrt{\sigma_{x}^{2}\left(1-\alpha_{0}\right)+\sigma_{z}^{2}}}\right)}{1-\Phi\left(\frac{\underline{\underline{z}}_{0}}{\sqrt{\sigma_{x}^{2}\left(1-\alpha_{0}\right)+\sigma_{z}^{2}}}\right.} \\
& =\left(\exp \left(p_{0}\right)-\exp \left(c_{0}^{*}\right)\right) \exp \left(-\gamma-b p_{0} \frac{1}{2}\left(\sigma_{x}^{2}+\sigma_{z}^{2}\right)\right)\left(\Phi\left(\frac{\alpha_{t \mid 0}\left(\sigma_{x}^{2}\left(1-\alpha_{0}\right)+\sigma_{z}^{2}\right)-\underline{\mathrm{z}}_{0}}{\sqrt{\sigma_{x}^{2}\left(1-\alpha_{0}\right)+\sigma_{z}^{2}}}\right) \exp \left(\alpha_{0} \widetilde{z}_{0}\right)+\Phi\left(\frac{\underline{\mathrm{z}}_{0}}{\sqrt{\sigma_{x}^{2}\left(1-\alpha_{0}\right)+\sigma_{z}^{2}}}\right)\right)
\end{aligned}
$$


while $E_{t-1}\left(\tilde{V} \mid p_{t} \neq p_{0}\right)=$

$$
\begin{aligned}
& =P\left(\widehat{z}_{t}<\bar{z}\left(p_{t}\right)\right)\left(\exp \left(p_{0}\right)-\exp \left(c_{0}^{*}\right)\right) \exp \left(-\gamma-b p_{0}+\alpha_{0} \widehat{z}_{0}+\frac{1}{2}\left(\sigma_{x}^{2}\left(1-\alpha_{0}\right)+\sigma_{z}^{2}\right)\right) \\
& +P\left(\widehat{z}_{t} \geq \bar{z}\left(p_{t}\right)\right)\left(\exp \left(p_{t}\right)-\exp \left(c_{0}^{*}\right)\right) \exp \left(-\gamma-b p_{t}+\frac{1}{2}\left(\sigma_{x}^{2}\left(1-\alpha_{t}\right)+\sigma_{z}^{2}\right)\right) E\left(\exp \left(\alpha_{t} \widehat{z}_{t}\right) \mid \widehat{z}_{t}>\bar{z}\left(p_{t}\right)\right) \\
& =\Phi\left(\frac{\bar{z}\left(p_{t}\right)}{\sqrt{\left(\sigma_{x}^{2}+\sigma_{z}^{2}\right)}}\right)\left(\exp \left(p_{0}\right)-\exp \left(c_{0}^{*}\right)\right) \exp \left(-\gamma-b p_{0}+\alpha_{0} \widehat{z}_{0}+\frac{1}{2}\left(\sigma_{x}^{2}\left(1-\alpha_{0}\right)+\sigma_{z}^{2}\right)\right) \\
& \left.+\Phi\left(\frac{\alpha_{t}\left(\sigma_{x}^{2}+\sigma_{z}^{2}\right)-\bar{z}\left(p_{t}\right)}{\sqrt{\left(\sigma_{x}^{2}+\sigma_{z}^{2}\right)}}\right)\left(\exp \left(p_{t}\right)-\exp \left(c_{0}^{*}\right)\right) \exp \left(-\gamma-b p_{t}+\frac{1}{2}\left(\sigma_{x}^{2}+\sigma_{z}^{2}\right)\right)\right)
\end{aligned}
$$

where we use the fact that the firm perceives $\widehat{z}_{t} \sim N\left(0, \hat{\sigma}_{t-1}^{2}\left(p_{t}\right)+\sigma_{z}^{2}\right)$, and $\Phi($.$) denotes the CDF$ of the standard normal distribution.

The first question of interest is if and when the expected continuation value is discontinuous at $p_{t}=p_{0}$. To answer this question, we evaluate the ratio $\frac{E_{t-1}\left(\tilde{V} \mid p_{1}=p_{0}\right)}{\lim _{p_{1} \rightarrow p_{0}} E_{t-1}\left(\tilde{V} \mid p_{1} \neq p_{0}\right)}$. It is useful to first evaluate the denominator and collect terms, concluding that $\lim _{p_{t} \rightarrow p_{0}} E_{t-1}\left(\tilde{V} \mid p_{t} \neq p_{0}\right)=$

$$
=\left(\exp \left(p_{0}\right)-\exp \left(c_{0}^{*}\right)\right) \exp \left(-\gamma-b p_{0}+\frac{1}{2}\left(\sigma_{x}^{2}+\sigma_{z}^{2}\right)\right)\left(\Phi\left(\frac{\bar{z}\left(p_{t}\right)}{\sqrt{\left(\sigma_{x}^{2}+\sigma_{z}^{2}\right)}}\right) \exp \left(\alpha_{0} \widetilde{z}_{0}\right)+\Phi\left(\frac{\alpha_{t}\left(\sigma_{x}^{2}+\sigma_{z}^{2}\right)-\bar{z}\left(p_{t}\right)}{\sqrt{\left(\sigma_{x}^{2}+\sigma_{z}^{2}\right)}}\right)\right)
$$

It then follows that the ratio $\frac{E_{t-1}\left(\tilde{V} \mid p_{t}=p_{0}\right)}{\lim _{p_{t} \rightarrow p_{0}} E_{t-1}\left(\tilde{V} \mid p_{t} \neq p_{0}\right)}=$

$$
=\frac{\Phi\left(\frac{\frac{\sigma_{x}^{2}}{2}\left(1-\alpha_{0}\right)+\frac{\alpha_{0}}{\alpha_{t \mid 0}} \widetilde{z}_{0}}{\sqrt{\sigma_{x}^{2}\left(1-\alpha_{0}\right)+\sigma_{z}^{2}}}\right) \exp \left(\alpha_{0} \widetilde{z}_{0}\right)+\Phi\left(\frac{\left(1-\alpha_{0}\right) \frac{\sigma_{x}^{2}}{2}-\frac{\alpha_{0}}{\alpha_{t \mid 0}} \widetilde{z}_{0}}{\sqrt{\sigma_{x}^{2}\left(1-\alpha_{0}\right)+\sigma_{z}^{2}}}\right)}{\Phi\left(\frac{\frac{\alpha_{0}}{\alpha_{t}} \widetilde{z}_{0}+\frac{\sigma_{x}^{2}}{2}}{\sqrt{\left(\sigma_{x}^{2}+\sigma_{z}^{2}\right)}}\right) \exp \left(\alpha_{0} \widetilde{z}_{0}\right)+\Phi\left(\frac{\frac{\sigma_{x}^{2}}{2}-\frac{\alpha_{0}}{\alpha_{t}} \widetilde{z}_{0}}{\sqrt{\left(\sigma_{x}^{2}+\sigma_{z}^{2}\right)}}\right)}
$$

where we have substituted in the respective values of the thresholds $\underline{\mathrm{z}}_{0}$ and $\bar{z}\left(p_{t}\right)$. The ratio limits to 1 as $\widetilde{z}_{0} \rightarrow \infty$, and it is below 1 at $\widetilde{z}_{0}=0$, as in this case

$$
\frac{E_{t-1}\left(\tilde{V} \mid p_{t}=p_{0}\right)}{\lim _{p_{t} \rightarrow p_{0}} E_{t-1}\left(\tilde{V} \mid p_{t} \neq p_{0}\right)}=\frac{\Phi\left(\frac{\frac{\sigma_{x}^{2}}{2}\left(1-\alpha_{0}\right)}{\sqrt{\sigma_{x}^{2}\left(1-\alpha_{0}\right)+\sigma_{z}^{2}}}\right)}{\Phi\left(\frac{\sigma_{x}^{2}}{2 \sqrt{\sigma_{x}^{2}+\sigma_{z}^{2}}}\right)}<1
$$

Next, we show that the derivative of the ratio in respect to $\widetilde{z}_{0}$ is positive for the relevant values $\widetilde{z}_{0} \geq 0$, which is enough to conclude that $\frac{E_{t-1}\left(\tilde{V} \mid p_{t}=p_{0}\right)}{\lim _{p_{t} \rightarrow p_{0}} E_{t-1}\left(\tilde{V} \mid p_{t} \neq p_{0}\right)}$ converges to 1 from below and hence is less than one for all finite $\widetilde{z}_{0} \geq 0$. The needed derivative,

$$
\frac{\partial \frac{E_{t-1}\left(\tilde{V} \mid p_{t}=p_{0}\right)}{\lim _{p_{t} \rightarrow p_{0}} E\left(\tilde{V} \mid p_{t} \neq p_{0}\right)}}{\partial \widetilde{z}_{0}}
$$


it is proportional to

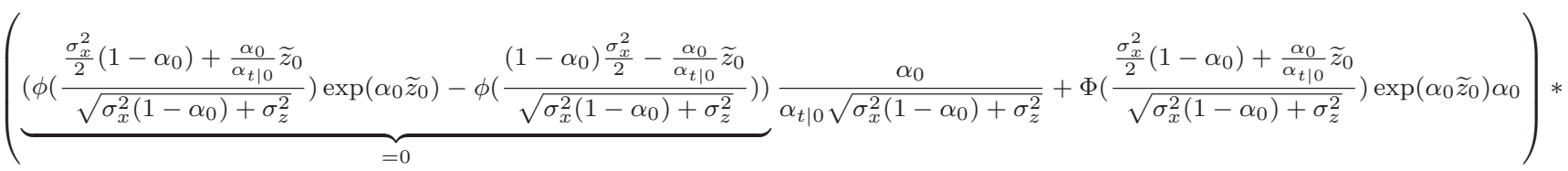

$$
\begin{aligned}
& \left(\Phi\left(\frac{\frac{\alpha_{0}}{\alpha_{t}} \widetilde{z}_{0}+\frac{\sigma_{x}^{2}}{2}}{\sqrt{\left(\sigma_{x}^{2}+\sigma_{z}^{2}\right)}}\right) \exp \left(\alpha_{0} \widetilde{z}_{0}\right)+\Phi\left(\frac{\frac{\sigma_{x}^{2}}{2}-\frac{\alpha_{0}}{\alpha_{t}} \widetilde{z}_{0}}{\sqrt{\left(\sigma_{x}^{2}+\sigma_{z}^{2}\right)}}\right)\right)-\left(\Phi\left(\frac{\frac{\sigma_{x}^{2}}{2}\left(1-\alpha_{0}\right)+\frac{\alpha_{0}}{\alpha_{t \mid 0}} \widetilde{z}_{0}}{\sqrt{\sigma_{x}^{2}\left(1-\alpha_{0}\right)+\sigma_{z}^{2}}}\right) \exp \left(\alpha_{0} \widetilde{z}_{0}\right)+\Phi\left(\frac{\left(1-\alpha_{0}\right) \frac{\sigma_{x}^{2}}{2}-\frac{\alpha_{0}}{\alpha_{t \mid 0}} \widetilde{z}_{0}}{\sqrt{\sigma_{x}^{2}\left(1-\alpha_{0}\right)+\sigma_{z}^{2}}}\right)\right) * \\
& (\underbrace{\left(\phi\left(\frac{\sigma_{x}^{2}}{\sqrt{\sigma_{x}^{2}+\sigma_{z}^{2}}}\right) \exp \left(\alpha_{0} \widetilde{z}_{0}\right)-\phi\left(\frac{\frac{\sigma_{x}^{2}}{2}-\frac{\alpha_{0}}{\alpha_{t}} \widetilde{z}_{0}}{\sqrt{\sigma_{x}^{2}+\sigma_{z}^{2}}}\right)\right) \frac{\alpha_{0}}{\alpha_{1} \sqrt{\sigma_{x}^{2}+\sigma_{z}^{2}}}}_{=0}+\Phi\left(\frac{\frac{\sigma_{x}^{2}}{2}+\frac{\alpha_{0}}{\alpha_{1}} \widetilde{z}_{0}}{\sqrt{\sigma_{x}^{2}+\sigma_{z}^{2}}}\right) \exp \left(\alpha_{0} \widetilde{z}_{0}\right) \alpha_{0}) \\
& =\alpha_{0} \exp \left(\alpha_{0} \widetilde{z}_{0}\right)\left[\Phi\left(\frac{\frac{\sigma_{x}^{2}}{2}\left(1-\alpha_{0}\right)+\frac{\alpha_{0}}{\alpha_{t \mid 0}} \widetilde{z}_{0}}{\sqrt{\sigma_{x}^{2}\left(1-\alpha_{0}\right)+\sigma_{z}^{2}}}\right) \Phi\left(\frac{\frac{\sigma_{x}^{2}}{2}-\frac{\alpha_{0}}{\alpha_{t}} \widetilde{z}_{0}}{\sqrt{\sigma_{x}^{2}+\sigma_{z}^{2}}}\right)-\Phi\left(\frac{\frac{\sigma_{x}^{2}}{2}\left(1-\alpha_{0}\right)-\frac{\alpha_{0}}{\alpha_{t \mid 0}} \widetilde{z}_{0}}{\sqrt{\sigma_{x}^{2}\left(1-\alpha_{0}\right)+\sigma_{z}^{2}}}\right) \Phi\left(\frac{\frac{\sigma_{x}^{2}}{2}+\frac{\alpha_{0}}{\alpha_{t}} \widetilde{z}_{0}}{\sqrt{\sigma_{x}^{2}+\sigma_{z}^{2}}}\right)\right]
\end{aligned}
$$

Thus, the derivative is positive if and only if

$$
\frac{\Phi\left(\frac{\frac{\sigma_{x}^{2}}{2}-\frac{\alpha_{0}}{\alpha_{t}} \widetilde{z}_{0}}{\sqrt{\sigma_{x}^{2}+\sigma_{z}^{2}}}\right)}{\Phi\left(\frac{\frac{\sigma_{x}^{2}}{2}+\frac{\alpha_{0}}{\alpha_{t}} \widetilde{z}_{0}}{\sqrt{\sigma_{x}^{2}+\sigma_{z}^{2}}}\right)}>\frac{\Phi\left(\frac{\frac{\sigma_{x}^{2}}{2}\left(1-\alpha_{0}\right)-\frac{\alpha_{0}}{\alpha_{t \mid 0}} \widetilde{z}_{0}}{\sqrt{\sigma_{x}^{2}\left(1-\alpha_{0}\right)+\sigma_{z}^{2}}}\right)}{\Phi\left(\frac{\frac{\sigma_{x}^{2}}{2}\left(1-\alpha_{0}\right)+\frac{\alpha_{0}}{\alpha_{t \mid 0}} \widetilde{z}_{0}}{\sqrt{\sigma_{x}^{2}\left(1-\alpha_{0}\right)+\sigma_{z}^{2}}}\right)}
$$

This inequality holds since

$$
\frac{\Phi\left(\frac{\frac{\sigma_{x}^{2}}{2}-\frac{\alpha_{0}}{\alpha_{t}} \widetilde{z}_{0}}{\sqrt{\sigma_{x}^{2}+\sigma_{z}^{2}}}\right)}{\Phi\left(\frac{\frac{\sigma_{x}^{2}}{2}+\frac{\alpha_{0}}{\alpha_{t}} \widetilde{z}_{0}}{\sqrt{\sigma_{x}^{2}+\sigma_{z}^{2}}}\right)}>\frac{\left.\frac{\frac{\sigma_{x}^{2}}{2}-\frac{\alpha_{0}}{\alpha_{t \mid 0}} \widetilde{z}_{0}}{\sqrt{\sigma_{x}^{2}+\sigma_{z}^{2}}}\right)}{\Phi\left(\frac{\frac{\sigma_{x}^{2}}{2}+\frac{\alpha_{0}}{\alpha_{t \mid 0}} \widetilde{z}_{0}}{\sqrt{\sigma_{x}^{2}+\sigma_{z}^{2}}}\right)}>\frac{\Phi\left(\frac{\frac{\sigma_{x}^{2}}{2}\left(1-\alpha_{0}\right)-\frac{\alpha_{0}}{\alpha_{t \mid 0}} \widetilde{z}_{0}}{\sqrt{\sigma_{x}^{2}\left(1-\alpha_{0}\right)+\sigma_{z}^{2}}}\right)}{\Phi\left(\frac{\frac{\sigma_{x}^{2}}{2}\left(1-\alpha_{0}\right)+\frac{\alpha_{0}}{\alpha_{t \mid 0}} \widetilde{z}_{0}}{\sqrt{\sigma_{x}^{2}\left(1-\alpha_{0}\right)+\sigma_{z}^{2}}}\right)}
$$

where the first inequality follows from $\alpha_{t \mid 0}<\alpha_{t}$, and the second from the fact that

$$
\frac{\partial \frac{\frac{\sigma_{x}^{2}}{2}\left(1-\tilde{\alpha}_{0}\right)-\frac{\alpha_{0}}{\alpha_{t \mid 0}} \widetilde{z}_{0}}{\sqrt{\sigma_{x}^{2}\left(1-\tilde{\alpha}_{0}\right)+\sigma_{z}^{2}}}}{\partial \tilde{\alpha}_{0}}<\frac{\partial \frac{\frac{\sigma_{x}^{2}}{2}\left(1-\tilde{\alpha}_{0}\right)+\frac{\alpha_{0}}{\alpha_{t \mid 0}} \widetilde{z}_{0}}{\sqrt{\sigma_{x}^{2}\left(1-\tilde{\alpha}_{0}\right)+\sigma_{z}^{2}}}}{\partial \tilde{\alpha}_{0}}
$$

and the fact that the term

$$
\frac{\partial\left(\frac{\Phi\left(\frac{\frac{\sigma_{x}^{2}}{2}\left(1-\tilde{\alpha}_{0}\right)-\frac{\alpha_{0}}{\alpha_{t \mid 0}} \tilde{z}_{0}}{\sqrt{\sigma_{x}^{2}\left(1-\tilde{\alpha}_{0}\right)+\sigma_{z}^{2}}}\right)}{\Phi\left(\frac{\frac{\sigma_{x}^{2}}{2}\left(1-\tilde{\alpha}_{0}\right)+\frac{\alpha_{0}}{\alpha_{t \mid 0}} \tilde{z}_{0}}{\sqrt{\sigma_{x}^{2}\left(1-\tilde{\alpha}_{0}\right)+\sigma_{z}^{2}}}\right)}\right)}{\partial \tilde{\alpha}_{0}}
$$


equals

$\phi\left(\frac{\frac{\sigma_{x}^{2}}{2}\left(1-\alpha_{0}\right)-\frac{\alpha_{0}}{\alpha_{t \mid 0}} \widetilde{z}_{0}}{\sqrt{\sigma_{x}^{2}\left(1-\alpha_{0}\right)+\sigma_{z}^{2}}}\right) \Phi\left(\frac{\frac{\sigma_{x}^{2}}{2}\left(1-\alpha_{0}\right)+\frac{\alpha_{0}}{\alpha_{t \mid 0}} \widetilde{z}_{0}}{\sqrt{\sigma_{x}^{2}\left(1-\alpha_{0}\right)+\sigma_{z}^{2}}}\right) \frac{\partial \frac{\frac{\sigma_{x}^{2}}{2}\left(1-\tilde{\alpha}_{0}\right)-\frac{\alpha_{0}}{\alpha_{t \mid 0}} \widetilde{z}_{0}}{\sqrt{\sigma_{x}^{2}\left(1-\tilde{\alpha}_{0}\right)+\sigma_{z}^{2}}}}{\partial \tilde{\alpha}_{0}}-\Phi\left(\frac{\frac{\sigma_{x}^{2}}{2}\left(1-\alpha_{0}\right)-\frac{\alpha_{0}}{\alpha_{t \mid 0}} \widetilde{z}_{0}}{\sqrt{\sigma_{x}^{2}\left(1-\alpha_{0}\right)+\sigma_{z}^{2}}}\right) \phi\left(\frac{\frac{\sigma_{x}^{2}}{2}\left(1-\alpha_{0}\right)+\frac{\alpha_{0}}{\alpha_{t \mid 0}} \widetilde{z}_{0}}{\sqrt{\sigma_{x}^{2}\left(1-\alpha_{0}\right)+\sigma_{z}^{2}}}\right) \frac{\partial \frac{\frac{\sigma_{x}^{2}}{2}\left(1-\tilde{\alpha}_{0}\right)+\frac{\alpha_{0}}{\alpha_{t \mid 0}} \widetilde{z}_{0}}{\sqrt{\sigma_{x}^{2}\left(1-\tilde{\alpha}_{0}\right)+\sigma_{z}^{2}}}}{\partial \tilde{\alpha}_{0}}$
$<0$

Thus, we can conclude that

$$
\frac{E_{t-1}\left(\tilde{V} \mid p_{t}=p_{0}\right)}{\lim _{p_{t} \rightarrow p_{0}} E_{t-1}\left(\tilde{V} \mid p_{t} \neq p_{0}\right)}<1
$$

for all $\widetilde{z}_{0} \geq 0$ meaning that there is discontinuous jump down in the continuation value at $p_{t}=p_{0}$.

Lastly, consider what value of $p_{t}$ optimizes the expected continuation value. Since the discontinuity at $p_{0}$ (the only potential corner solution) is a jump down, the maximizing $p_{t}$ must be the interior maximum, which satisfies the FOC condition that $\frac{\partial E_{t-1}\left(\tilde{V} \mid p_{t} \neq p_{0}\right)}{\partial p_{t}}=0$. Taking the derivative, $\frac{\partial E_{t-1}\left(\tilde{V} \mid p_{t} \neq p_{0}\right)}{\partial p_{t}}=$

$$
\begin{aligned}
& =\phi\left(\frac{\bar{z}\left(p_{t}\right)}{\sqrt{\sigma_{x}^{2}+\sigma_{z}^{2}}}\right)\left(e^{p_{0}}-e^{c_{0}^{*}}\right) \exp \left(-\gamma-b p_{0}+\alpha_{0} \widehat{z}_{0}+\frac{1}{2}\left(\sigma_{x}^{2}\left(1-\alpha_{0}\right)+\sigma_{z}^{2}\right)\right) \frac{\frac{\partial \bar{z}\left(p_{t}\right)}{\partial p_{t}}}{\sqrt{\sigma_{x}^{2}+\sigma_{z}^{2}}} \\
& -\phi\left(\frac{\alpha_{t}\left(\sigma_{x}^{2}+\sigma_{z}^{2}\right)-\bar{z}\left(p_{t}\right)}{\sqrt{\left(\sigma_{x}^{2}+\sigma_{z}^{2}\right)}}\right)\left(e^{p_{t}}-e^{c_{0}^{*}}\right) \exp \left(-\gamma-b p_{t}+\frac{1}{2}\left(\sigma_{x}^{2}\left(1-\alpha_{t}\right)+\sigma_{z}^{2}+\alpha_{t}^{2}\left(\sigma_{x}^{2}+\sigma_{z}^{2}\right)\right)\right) \frac{\frac{\partial \bar{z}\left(p_{t}\right)}{\partial p_{t}}}{\sqrt{\sigma_{x}^{2}+\sigma_{z}^{2}}} \\
& +\Phi\left(\frac{\alpha_{t}\left(\sigma_{x}^{2}+\sigma_{z}^{2}\right)-\bar{z}\left(p_{t}\right)}{\sqrt{\left(\sigma_{x}^{2}+\sigma_{z}^{2}\right)}}\right) \exp \left(-\gamma-b p_{t}+\frac{1}{2}\left(\sigma_{x}^{2}\left(1-\alpha_{t}\right)+\sigma_{z}^{2}+\alpha_{t}^{2}\left(\sigma_{x}^{2}+\sigma_{z}^{2}\right)\right)\right)\left(e^{p_{t}}-b\left(e^{p_{t}}-e^{c_{0}^{*}}\right)\right)
\end{aligned}
$$

The above expression limits to zero as $p_{t} \rightarrow p_{0}$. To see that, note that $\lim _{p_{t} \rightarrow p_{0}} \frac{\partial \bar{z}\left(p_{t}\right)}{\partial p_{t}}=0$, thus the first 2 terms of the FOC expression above fall out. For the last term, using $p_{0}=\ln \left(\frac{b}{b-1}\right)+c_{0}$ it follows that

$$
\left(e^{p_{0}}-b\left(e^{p_{0}}-e^{c_{0}^{*}}\right)\right)=\frac{b}{b-1} e^{c_{0}^{*}}-\frac{b}{b-1} e^{c_{0}^{*}}=0
$$

Therefore, we can conclude that $\lim _{p_{t} \rightarrow p_{0}} \frac{\partial E_{t-1}\left(\tilde{V} \mid p_{t} \neq p_{0}\right)}{\partial p_{t}}=0$, and thus the interior maximum of the expected continuation value is $p_{t} \rightarrow p_{0}$.

Intuitively, $p_{t}^{*}=\arg \min _{p}\left(p-p_{0}\right)^{2}$ s.t. $p \neq p_{0}$, ensures that the new signal $y_{t}$ will be informative about a price as close as possible to the ex-ante expected optimal $p_{0}$, and thus achieves almost the same markup - this makes the new information highly relevant. As a result, if the realization of $\widehat{z}_{t}$ happens to be good enough, i.e. $\widehat{z}_{t}$ is above a threshold $\bar{z}_{t}\left(p_{t}^{*}\right)$ that is characterized in the proof above, then the firm will stick with this price in the future, set $p_{t+k}=p_{t}^{*}$, and take advantage of the unexpectedly high demand at that price. On the other hand, if the signal realization happens to be bad, the firm can safely switch back to the ex-ante optimal $p_{0}$, where the belief about demand is not affected by $\widehat{z}_{t}$, and still offers lower uncertainty and a good perceived markup. 
The reason for not picking $p_{t}=p_{0}$ is that a bad signal realization at $p_{0}$ erodes the ex-ante best available pricing option, $p_{0}$, and at the same time the firm does not have a good fall-back alternative, as it has no observations of demand at other prices. If in that case the realization of $\widehat{z}_{t}$ falls below the threshold $\underline{z}_{0}$, the news about $x\left(p_{0}\right)$ is bad enough to incentivize the firm to set $p_{t+k}$ to a previously unvisited price. Due to this downside risk at $p_{0}$, there is a first-order gain of obtaining information at a new price, which manifests in the discontinuous jump down in the expected continuation value at $p_{0}$.

As shown in Proposition 3, the best forward-looking strategy is therefore to experiment by posting a new price. This exploration incentive could potentially overturn the rigidity result implied by the static maximization pricing choice analyzed earlier, but as we show next it turns out that this results is specific to the firm having seen only one price in the past. In more general situations, when the firm has seen more than one distinct price point in the past, forward-looking behavior can in fact reinforce the static rigidity incentives.

\section{Exploration makes prices stickier, when $\varepsilon^{t}$ contains observations at multiple prices}

Proposition 4. There is a non-singleton interval of costs $(\underline{c}, \bar{c})$ around $c_{0}^{*}$, and a threshold $\chi>0$, such that if $\widehat{z}>\chi$, then for any $c \in(\underline{c}, \bar{c})$ :

$$
p_{0}=\arg \max _{p_{t}} E\left[\tilde{V}\left(\left\{\varepsilon^{1}, p_{t}, y_{t}\right\}, c\right) \mid \varepsilon^{1}, p_{t}\right] .
$$

Moreover, the threshold $\chi$ is decreasing in $\left|p_{1}-p_{0}\right|$.

Proof. The proof follows a similar logic as the previous one. First, we characterize the optimal $p_{t+k}$ for $c=c_{0}^{*}$, but now conditional on $\varepsilon^{1}$, and then use it to compute the expected continuation value and show that it is maximized at $p_{t}=p_{0}$. Lastly, we appeal to continuity to conclude that $p_{t}=p_{0}$ is optimal for an interval of cost values around $c_{0}^{*}$. In addition to the signal-to-noise ratio notation $\alpha_{0}, \alpha_{t \mid 0}, \alpha_{t}$ defined in the previous proof, we define

$$
\begin{gathered}
\alpha_{1} \equiv \alpha_{t-1}\left(p_{1} ; p_{1}\right)=\frac{\sigma_{x}^{2}}{\sigma_{x}^{2}+\sigma_{z}^{2} / N_{1}} \\
\alpha_{t \mid 1} \equiv \alpha_{t}\left(p_{1} ; p_{1} \mid p_{t}=p_{1}\right)=\frac{\sigma_{x}^{2}}{\sigma_{x}^{2}\left(N_{1}+1\right)+\sigma_{z}^{2}}
\end{gathered}
$$

Similarly, we define the (variance corrected) innovation in the signal at $p_{1}$ as

$$
\widetilde{z}_{1} \equiv \widehat{z}_{1}-\frac{1}{2} \sigma_{x}^{2}=y_{1}-\left(-\gamma-b p_{1}\right)-\frac{1}{2} \sigma_{x}^{2}
$$

The optimal policy at $t+k$ follows a similar structure to the one described in the previous proof. Conditional on just $\varepsilon^{1}$ the optimal $p_{t+k}$ is equal to $p_{0}$, and the way the new information contained in $y_{t}$ affects the optimal $p_{t+k}$ depends on the position of $p_{t}$. If $p_{t}=p_{0}$, then the firm stays at $p_{0}$ 
unless the new signal is too bad $\left(\widehat{z}_{t}<\underline{z}_{0}\right)$. If $p_{t}=p_{1}$, then the firm moves to $p_{1}$ if the signal is $\operatorname{good}$ enough $\left(\widehat{z}_{t}>\bar{z}_{1}\right)$ otherwise stays at $p_{0}$. And if $p_{t} \notin\left\{p_{0}, p_{1}\right\}$, then the firm again stays at $p_{0}$ unless the signal is too good, but compared to a different threshold: $\widehat{z}_{t}>\bar{z}\left(p_{t}\right)$. The key difference from the previous proof is what happens if $p_{t}=p_{0}$ and the signal is sufficiently bad to prompt a move $\left(\widehat{z}_{t}<\underline{z}_{0}\right)$. There exists a $\chi_{1}>0$ such that if $\widehat{z}_{1}>\chi_{1}$, then the firm does not move to the interior optimum $p^{i n t}$, but rather to $p_{1}$, which as another relatively good price at which the firm has built some information capital is a better option than the brand new $p^{\text {int }}$ where the firm has not accumulated any information. To see this, note that

$$
\frac{E\left(\nu_{t+k}^{*}\left(p_{1}, c_{0}^{*}\right) \mid \varepsilon^{1}, p_{t}=p_{0}\right)}{\lim _{p \rightarrow p_{0}} E\left(\nu_{t+k}^{*}\left(p, c_{0}^{*}\right) \mid \varepsilon^{1}, p_{t}=p_{0}\right)}=\left(b \exp \left(p_{1}-p_{0}\right)-b+1\right) \exp \left(-b\left(p_{1}-p_{0}\right)+\alpha_{1} \widetilde{z}_{1}\right)>1
$$

Note that the RHS is increasing in $\widetilde{z}_{1}$, and thus in $\widehat{z}_{1}$ and limits to infinity as $\widehat{z}_{1} \rightarrow \infty$, hence there exists a constant $\chi_{1}>0$ such that the above ratio is strictly greater than one when $\widehat{z}>\chi_{1}$. For the rest of the proof we assume that $\widehat{z}_{1}>\chi_{1}$ so that the above inequality holds. The relevant thresholds $\underline{\mathrm{z}}_{0}, \bar{z}_{1}, \bar{z}\left(p_{t}\right)$ can be computed as before, by finding the value of the signal at which the firm is indifferent between $p_{0}$ and the respective alternative option:

$$
\begin{aligned}
\underline{z}_{0} & =\frac{\sigma_{x}^{2}}{2}\left(1-\alpha_{0}\right)-\frac{1}{\alpha_{t \mid 0}}\left(b\left(p_{1}-p_{0}\right)-\ln \left(b e^{\left(p_{1}-p_{0}\right)}-b+1\right)\right) \\
\bar{z}_{1} & =\frac{\sigma_{x}^{2}}{2}\left(1-\alpha_{1}\right)+\frac{1}{\alpha_{t \mid 1}}\left(b\left(p_{1}-p_{0}\right)-\ln \left(b e^{\left(p_{1}-p_{0}\right)}-b+1\right)\right) \\
\bar{z}\left(p_{t}\right) & =\frac{\alpha_{0}}{\alpha_{t}} \widetilde{z}_{0}+\frac{\sigma_{x}^{2}}{2}-\frac{1}{\alpha_{t}}\left[\ln \left(\frac{\exp \left(p_{t}\right)-\exp \left(c_{0}^{*}\right)}{\exp \left(p_{0}\right)-\exp \left(c_{0}^{*}\right)}\right)+b\left(p_{0}-p_{t}\right)\right]
\end{aligned}
$$

So the $t+k$ optimal pricing policy is:

$$
p_{t+1}^{*}= \begin{cases}p_{0} & \text { if } p_{t}=p_{0} \text { and } \widehat{z}_{t} \geq \underline{z}_{0}, \text { or } p_{t}=p_{1} \text { and } \widehat{z}_{t} \leq \bar{z}_{1} \text { or } p_{t} \notin\left\{p_{0}, p_{1}\right\} \text { and } \widehat{z}_{t} \leq \bar{z}\left(p_{t}\right) \\ p_{1} & \text { if } p_{t}=p_{1} \text { and } \widehat{z}_{t}>\bar{z}_{1} \text { or } p_{t}=p_{0} \text { and } \widehat{z}_{t}<\underline{z}_{0} \\ p_{t} & \text { if } p_{t} \notin\left\{p_{0}, p_{1}\right\} \text { and } \widehat{z}_{t}>\bar{z}\left(p_{t}\right)\end{cases}
$$

We can now use this result to characterize the expected continuation value and find its maximizer. Note that the value of $p_{t}$ that maximizes $E\left(\left[\tilde{V}\left(\left\{\varepsilon^{1}, p_{t}, y_{t}\right\}, c_{0}^{*}\right)\left|\varepsilon^{1}, p_{t}\right|\right]\right)$ is either one of the two corner solutions $p_{0}$ and $p_{1}$, or the interior maximum. Moreover, we can appeal to the proof of Proposition 3 for the result that the expected continuation value achieves its interior maximum at the limit of $p_{t} \rightarrow p_{0}$. This follows because under $\psi \rightarrow \infty$ the additional signal $y_{1}$ only matters when updating beliefs at $p_{1}$ itself, hence at $p \neq p_{1}$ the expected continuation value is equivalent to the one conditional on $\varepsilon^{0}$, that we analyzed above. We proceed in two steps. First we show that the two corner solutions are in fact equivalent to each other, and then we conclude by showing that 
$p_{0}$ also dominates the interior solution $p^{i n t}$. The expected value $E\left(\left[\tilde{V}\left(\left\{\varepsilon^{1}, p_{t}, y_{t}\right\}, c_{0}^{*} \mid \varepsilon^{1}, p_{t}=p_{0}\right)\right]\right)$ is slightly different than before, because the fall back option (in case of a bad new signal $y_{t}$ ) is now $p_{1}$. Now, $E_{t-1}\left(\tilde{V} \mid p_{t}=p_{0}\right)=$

$$
\begin{aligned}
& =\Phi\left(\frac{\underline{\mathrm{z}}_{0}}{\sqrt{\sigma_{x}^{2}\left(1-\alpha_{0}\right)+\sigma_{z}^{2}}}\right)\left(\exp \left(p_{1}\right)-\exp \left(c_{0}^{*}\right)\right) \exp \left(-\gamma-b p_{1}+\alpha_{1} \widehat{z}_{1}+\frac{1}{2}\left(\sigma_{x}^{2}\left(1-\alpha_{1}\right)+\sigma_{z}^{2}\right)\right) \\
& +\left(1-\Phi\left(\frac{\underline{\mathrm{z}}_{0}}{\sqrt{\sigma_{x}^{2}\left(1-\alpha_{0}\right)+\sigma_{z}^{2}}}\right)\right)\left(\exp \left(p_{0}\right)-\exp \left(c_{0}^{*}\right)\right) \exp \left(-\gamma-b p_{0}+\alpha_{0} \widehat{z}_{0}+\frac{1}{2}\left(\sigma_{x}^{2}\left(1-\alpha_{0}\right)+\sigma_{z}^{2}\right)\right) \frac{\Phi\left(\frac{\alpha_{t \mid 0}\left(\sigma_{x}^{2}\left(1-\alpha_{0}\right)+\sigma_{z}^{2}\right)-\underline{\mathrm{z}}_{0}}{\sqrt{\sigma_{x}^{2}\left(1-\alpha_{0}\right)+\sigma_{z}^{2}}}\right)}{1-\Phi\left(\frac{\underline{\underline{z}}_{0}}{\sqrt{\sigma_{x}^{2}\left(1-\alpha_{0}\right)+\sigma_{z}^{2}}}\right.} \\
& =\frac{1}{b-1} \exp \left(c_{0}^{*}-\gamma-b p_{0}+\alpha_{0} \widetilde{z}_{0}+\frac{1}{2}\left(\sigma_{x}^{2}+\sigma_{z}^{2}\right)\right)\left(\Phi\left(\frac{\alpha_{t \mid 0}\left(\sigma_{x}^{2}\left(1-\alpha_{0}\right)+\sigma_{z}^{2}\right)-\underline{\mathrm{z}}_{0}}{\sqrt{\sigma_{x}^{2}\left(1-\alpha_{0}\right)+\sigma_{z}^{2}}}\right)+\Phi\left(\frac{\underline{\mathrm{z}}_{0}}{\sqrt{\sigma_{x}^{2}\left(1-\alpha_{0}\right)+\sigma_{z}^{2}}}\right)\left(b e^{p_{1}-p_{0}}-b+1\right) e^{-b\left(p_{1}-p_{0}\right)}\right)
\end{aligned}
$$

Similarly, $E\left(\left[\tilde{V}\left(\left\{\varepsilon^{1}, p_{t}, y_{t}\right\}, c_{0}^{*} \mid \varepsilon^{1}, p_{t}=p_{1}\right)\right]\right)$ can be computed as $E_{t-1}\left(\tilde{V} \mid p_{t}=p_{1}\right)=$

$$
\begin{aligned}
& =P\left(\widehat{z}_{t} \leq \bar{z}_{1}\right)\left(\exp \left(p_{0}\right)-\exp \left(c_{0}^{*}\right)\right) \exp \left(-\gamma-b p_{0}+\alpha_{0} \widehat{z}_{0}+\frac{1}{2}\left(\sigma_{x}^{2}\left(1-\alpha_{0}\right)+\sigma_{z}^{2}\right)\right) \\
& +P\left(\widehat{z}_{t}>\bar{z}_{1}\right)\left(\exp \left(p_{1}\right)-\exp \left(c_{0}^{*}\right)\right) \exp \left(-\gamma-b p_{1}+\alpha_{1} \widehat{z}_{1}+\frac{1}{2}\left(\sigma_{x}^{2}\left(1-\alpha_{1}\right)\left(1-\alpha_{t \mid 1}\right)+\sigma_{z}^{2}\right)\right) E\left(\exp \left(\alpha_{t \mid 1} \widehat{z}_{t}\right) \mid \widehat{z}_{t}>\bar{z}_{1}\right) \\
& =\frac{1}{b-1} \exp \left(c_{0}^{*}-\gamma-b p_{0}+\alpha_{0} \widetilde{z}_{0}+\frac{1}{2}\left(\sigma_{x}^{2}+\sigma_{z}^{2}\right)\right)\left[\Phi\left(\frac{\bar{z}_{1}}{\sqrt{\left(\sigma_{x}^{2}\left(1-\alpha_{1}\right)+\sigma_{z}^{2}\right)}}\right)+\Phi\left(\frac{\alpha_{t \mid 1}\left(\sigma_{x}^{2}\left(1-\alpha_{1}\right)+\sigma_{z}^{2}\right)-\bar{z}_{1}}{\sqrt{\left(\sigma_{x}^{2}\left(1-\alpha_{1}\right)+\sigma_{z}^{2}\right)}}\right)\left(b e^{p_{1}-p_{0}}-b+1\right) e^{-b\left(p_{1}-p_{0}\right)}\right]
\end{aligned}
$$

Substituting in the expressions for $\underline{z}_{0}$ and $\bar{z}_{1}$ we obtain

$$
E_{t-1}\left(\tilde{V} \mid p_{t}=p_{0}\right)=E_{t-1}\left(\tilde{V} \mid p_{t}=p_{1}\right)
$$

Lastly, note that for $p_{t} \notin\left\{p_{0}, p_{1}\right\}, E\left(\left[\tilde{V}\left(c_{0},\left\{\varepsilon^{t-1}, p_{t}, y_{t}\right\} \mid \varepsilon^{1}, p_{t}\right)\right]\right)$ is the same as computed in the proof of Proposition 3 above. As a result, the interior maximum is achieved at $\lim p_{t} \rightarrow p_{0}$, hence to conclude our argument we need to compare $E_{t-1}\left(\tilde{V} \mid p_{t}=p_{0}\right)$ against $\lim _{p_{t} \rightarrow p_{0}} E_{t-1}\left(\tilde{V} \mid p_{t} \notin\left\{p_{0}, p_{1}\right\}\right)$, which in turn equals

$$
\left(\exp \left(p_{0}\right)-\exp \left(c_{0}^{*}\right)\right) \exp \left(-\gamma-b p_{0}+\frac{1}{2}\left(\sigma_{x}^{2}+\sigma_{z}^{2}\right)\right)\left(\Phi\left(\frac{\bar{z}\left(p_{t}\right)}{\sqrt{\left(\sigma_{x}^{2}+\sigma_{z}^{2}\right)}}\right) \exp \left(\alpha_{0} \widetilde{z}_{0}\right)+\Phi\left(\frac{\alpha_{t}\left(\sigma_{x}^{2}+\sigma_{z}^{2}\right)-\bar{z}\left(p_{t}\right)}{\sqrt{\left(\sigma_{x}^{2}+\sigma_{z}^{2}\right)}}\right)\right)
$$

Let $\hat{\theta}=\left(b\left(p_{1}-p_{0}\right)-\ln \left(b e^{\left(p_{1}-p_{0}\right)}-b+1\right)\right)>0$, then after substituting the expressions for $\underline{\mathrm{z}}_{0}$ and $\bar{z}\left(p_{t}\right)$ and simplifying, the ratio of the two expected continuation values simplifies to:

$$
\frac{E_{t-1}\left(\tilde{V} \mid p_{t}=p_{0}\right)}{\lim _{p_{t} \rightarrow p_{0}} E_{t-1}\left(\tilde{V} \mid p_{t} \notin\left\{p_{0}, p_{1}\right\}\right)}=\frac{\Phi\left(\frac{\frac{\sigma_{x}^{2}}{2}\left(1-\alpha_{0}\right)+\frac{\hat{\theta}}{\alpha_{t \mid 0}}}{\sqrt{\sigma_{x}^{2}\left(1-\alpha_{0}\right)+\sigma_{z}^{2}}}\right)+\Phi\left(\frac{\frac{\sigma_{x}^{2}}{2}\left(1-\alpha_{0}\right)-\frac{\hat{\theta}}{\alpha_{t \mid 0}}}{\sqrt{\sigma_{x}^{2}\left(1-\alpha_{0}\right)+\sigma_{z}^{2}}}\right) \exp (-\hat{\theta})}{\Phi\left(\frac{\frac{\alpha_{0}}{\alpha_{t}} \widetilde{z}_{0}+\frac{\sigma_{x}^{2}}{2}}{\sqrt{\left(\sigma_{x}^{2}+\sigma_{z}^{2}\right)}}\right)+\Phi\left(\frac{\frac{\sigma_{x}^{2}}{2}-\frac{\alpha_{0}}{\alpha_{t}} \widetilde{z}_{0}}{\sqrt{\left(\sigma_{x}^{2}+\sigma_{z}^{2}\right)}}\right) \exp \left(-\alpha_{0} \widetilde{z}_{0}\right)}
$$

The denominator is decreasing in $\widetilde{z}_{0}$ and thus also in $\widehat{z}_{0}$, hence for every $\hat{\theta}$ there is a $\widehat{z}_{0}$ big enough such that the above ratio is strictly greater than 0 . As a result, there exists a finite constant $\chi_{0}>0$ such that when $\widehat{z}_{0}>\chi_{0}$ it follows that $p_{t}=p_{0}$ maximizes the expected continuation value. 
Finally, let $\chi=\max \left\{\chi_{0}, \chi_{1}\right\}$, then if $\widehat{z}_{1}=\widehat{z}_{0}>\chi$,

$$
p_{0}=\arg \max _{p_{t}} E\left[\tilde{V}\left(\left\{\varepsilon^{1}, p_{t}, y_{t}\right\}, c_{0}^{*}\right) \mid \varepsilon^{1}, p_{t}\right]
$$

Since $\tilde{V}$ is continuous in the cost shock $c$, it follows that there exists a non-singleton interval $(\underline{\mathrm{c}}, \bar{c})$ around $c_{0}^{*}$, such that if $c \in(\underline{\mathrm{c}}, \bar{c})$, then

$$
p_{0}=\arg \max _{p_{t}} E\left[\tilde{V}\left(\left\{\varepsilon^{1}, p_{t}, y_{t}\right\}, c\right) \mid \varepsilon^{1}, p_{t}\right]
$$

Lastly, we want to show that $\frac{\partial \chi}{\partial\left|p_{0}-p_{1}\right|}<0$. This follows directly form the facts that (i) the numerator of (37) is decreasing in $\hat{\theta}$, and that (ii) $\hat{\theta}$ is increasing in $\left(p_{1}-p_{0}\right)$. Hence, as we decrease the distance between $p_{0}$ and $p_{1}$, we increase the RHS of (37), and thus we require a smaller $\widehat{z}=\widehat{z}_{0}=\widehat{z}$ to make the ratio bigger than 1 .

\section{A.4 Household problem}

The representative household consumes and works according to

$$
\max _{c_{t+k}, L_{i, t+k}} \sum_{k=0}^{\infty} E_{t}\left(\beta^{t+k}\left[c_{t+k}-\int L_{i, t+k} d i\right]\right)
$$

where $c_{t}$ denotes log consumption of the aggregate good, subject to the budget constraint

$$
\int e^{p_{j, t}+c_{j, t}} d j+E_{t} Q_{t+1} D_{t+1}=D_{t}+e^{p_{t}+w_{t}} \int L_{i, t} d i+\int v_{i, t} d i
$$

where $Q_{t+1}$ is the stochastic discount factor, $D_{t}$ are state contingent claims on the aggregate shocks, $v_{i, t}$ is the profit from the monopolistic intermediaries and $w_{t}$ is the log real wage. The optimal labor supply condition is simply $w_{t}=c_{t}$, while the market clearing states that $c_{t}=y_{t}$. Substituting the wage into the firm's profit we obtain equation (21).

\section{A.5 Proofs on learning and nominal rigidity}

Proposition A1. The nominal price $p_{i, 1}=\widetilde{p}_{j, 1}+\widetilde{r}_{i, 0}$ is a local maximizer of the worst-case expected profits for any aggregate price $p_{1} \in\left(\bar{p}_{1}+\ln \left(\frac{b}{b-1} \frac{b-\alpha \delta-1}{b-\alpha \delta}\right), \bar{p}_{1}+\ln \left(\frac{b}{b-1} \frac{b+\alpha \delta-1}{b+\alpha \delta}\right)\right)$.

Proof. Let $v^{*}\left(\varepsilon^{0}, s_{1}, p_{i, 1}\right)$ denote the worst-case expected profit, conditional on the history $\varepsilon^{0}$ and the current state $s_{1}=\left\{\omega_{i, 1}, p_{1}, y_{1}, \widetilde{p}_{j, 1}\right\}$, evaluated at some nominal price $p_{i, 1}$. Conditional on $p_{i, 1}-$ $\widetilde{p}_{j, 1}$, the worst-case beliefs are given by equations (25) and (26). Take a first-order approximation of the change in profits, $v^{*}\left(\varepsilon^{0}, s_{1}, p_{i, 1}\right)-v^{*}\left(\varepsilon^{0}, s_{1}, \widetilde{p}_{j, 1}+\widetilde{r}_{i, 0}\right)$, evaluated around $p_{i, 1}=\widetilde{p}_{j, 1}+\widetilde{r}_{i, 0}$. 
This equals

$$
\left[\frac{e^{\widetilde{p}_{j, 1}+\widetilde{r}_{i, 0}-p_{1}}}{e^{\widetilde{p}_{j, 1}+\widetilde{r}_{i, 0}-p_{1}}-e^{y_{1}-\omega_{i, 1}}}-\left(b+\alpha \delta^{*}\right)\right]\left(p_{i, 1}-\widetilde{p}_{j, 1}-\widetilde{r}_{i, 0}\right),
$$

where $\delta^{*}=\delta \operatorname{sgn}\left(p_{i, 1}-\widetilde{p}_{j, 1}-\widetilde{r}_{i, 0}\right)$.

It then follows that for any $p_{1} \in(\underline{p}, \bar{p})$, where we define

$$
\underline{p}=\bar{p}_{1}+\ln \left(\frac{b}{b-1} \frac{b-\alpha \delta-1}{b-\alpha \delta}\right) ; \bar{p}=\bar{p}_{1}+\ln \left(\frac{b}{b-1} \frac{b+\alpha \delta-1}{b+\alpha \delta}\right),
$$

we have

$$
\frac{e^{\widetilde{p}_{j, 1}+\widetilde{r}_{i, 0}-p_{1}}}{e^{\widetilde{p}_{j, 1}+\widetilde{r}_{i, 0}-p_{1}}-e^{y_{1}-\omega_{i, 1}}} \in(b-\alpha \delta, b+\alpha \delta),
$$

which makes the first-order derivative of the change in profits negative to the right of $\widetilde{p}_{j, 1}+\widetilde{r}_{i, 0}$ and positive to its left. This gives the necessary and sufficient conditions for $\widetilde{p}_{j, 1}+\widetilde{r}_{i, 0}$ to be a local maximizer.

Proposition A2. Let $\delta^{\text {index }}=\delta \operatorname{sgn}\left(p_{1}-\widetilde{p}_{j, 1}\right)$. Up to a first-order approximation around $p_{1}=\widetilde{p}_{j, 1}$, the difference $\ln v^{*}\left(\varepsilon^{0}, s_{1}, \widetilde{r}_{i, 0}+p_{1}\right)-\ln v^{*}\left(\varepsilon^{0}, s_{1}, \widetilde{r}_{i, 0}+\widetilde{p}_{j, 1}\right)$ equals

$$
\left[\frac{e^{\widetilde{r}_{i, 0}}}{e^{\widetilde{r}_{i, 0}}-e^{y_{1}-\omega_{i, 1}}}-b-\alpha \delta^{\text {index }}\right]\left(p_{1}-\widetilde{p}_{j, 1}\right)<0 .
$$

Proof. First, analyze the worst-case expected profit under a policy rule that implements indexation, i.e. $p_{i, 1}^{\text {index }}=\widetilde{r}_{i, 0}+p_{1}$, given by

$$
v^{*}\left(\varepsilon^{0}, s_{1}, p_{i, 1}^{\text {index }}\right)=\left(e^{\widetilde{r}_{i, 0}}-e^{y_{1}-\omega_{i, 1}}\right) e^{\widehat{x}_{0}^{*}\left(p_{i, 1}^{\text {index }}, y_{1}, p_{1}, \widetilde{p}_{j, 1}\right)}
$$

where $\widehat{x}_{0}^{*}\left(p_{i, 1}^{\text {index }}, y_{1}, p_{1}, \widetilde{p}_{j, 1}\right)$ equals $.5\left(\widehat{\sigma}_{0}^{2}+\sigma_{z}^{2}\right)+c_{t}-b \widetilde{r}_{i, 0}-\gamma+\alpha\left[y_{0}-\left(-\gamma-b \widetilde{r}_{i, 0}\right)\right]$ plus

$$
\min _{\delta^{\prime} \in[-\delta, \delta]} \min _{\phi\left(p_{t}-\widetilde{p}_{j, t}\right) \in\left[-\gamma_{p}, \gamma_{p}\right]}-\alpha \delta^{\prime}\left(p_{1}-\widetilde{p}_{j, 1}\right)+\alpha \delta^{\prime}\left[\phi\left(p_{1}-\widetilde{p}_{j, 1}\right)-\phi\left(p_{0}-\widetilde{p}_{j, 0}\right)\right]
$$

The joint worst-case demand shape and co-integrating relationship are given by

$$
\delta^{\text {index }}=\delta \operatorname{sgn}\left(p_{1}-\widetilde{p}_{j, 1}\right) ; \phi^{\text {index }}\left(p_{1}-\widetilde{p}_{j, 1}\right)-\phi^{\text {index }}\left(p_{0}-\widetilde{p}_{j, 0}\right)=-2 \gamma_{p} \operatorname{sgn}\left(p_{1}-\widetilde{p}_{j, 1}\right) .
$$

Given the presence of the kink we compute a $\log$-linear approximation of $v^{*}\left(\varepsilon^{0}, s_{1}, p_{i, 1}^{\text {index }}\right)$ around $p_{1}=\widetilde{p}_{j, 1}$. At its right we have

$$
\frac{d \ln v^{*}\left(\varepsilon^{0}, s_{1}, p_{i, 1}^{\text {index }}\right)}{d p_{1}}=-\alpha \delta
$$

while at its left, the derivative is

$$
\frac{d \ln v^{*}\left(\varepsilon^{0}, s_{1}, p_{i, 1}^{\text {index }}\right)}{d p_{1}}=\alpha \delta
$$


The constant term in the approximation is given by evaluating $\ln v^{*}\left(\varepsilon^{0}, s_{1}, p_{i, 1}^{\text {index }}\right)$ at $p_{1}=\widetilde{p}_{j, 1}$ :

$$
\ln \left(e^{\widetilde{r}_{i, 1}^{*}}-e^{y_{1}-\omega_{i, 1}}\right)+c_{t}-b \widetilde{r}_{i, 0}-\gamma+\alpha\left[y_{0}-\left(-\gamma-b \widetilde{r}_{i, 0}\right)\right]-2 \alpha \delta \gamma_{p}
$$

Second, let us analyze the worst-case expected profit under the original policy, $p_{i, 1}^{*}=\widetilde{r}_{i, 0}+\widetilde{p}_{j, 1}$, which targets the same $\widetilde{r}_{i, 0}$ but by adjusting the nominal price to the review signal $\widetilde{p}_{j, 1}$. We have

$$
v^{*}\left(\varepsilon^{0}, s_{1}, p_{i, 1}^{*}\right)=\left(e^{\widetilde{r}_{i, 0}+\widetilde{p}_{j, 1}-p_{1}}-e^{y_{1}-\omega_{i, 1}}\right) e^{\widehat{x}_{0}^{*}\left(p_{i, 1}^{*}, y_{1}, p_{1}, \widetilde{p}_{j, 1}\right)}
$$

where $\widehat{x}_{0}^{*}\left(p_{i, 1}^{*}, y_{1}, p_{1}, \widetilde{p}_{j, 1}\right)$ equals $.5\left(\widehat{\sigma}_{0}^{2}+\sigma_{z}^{2}\right)+c_{t}-b\left(\widetilde{r}_{i, 0}+\widetilde{p}_{j, 1}-p_{1}\right)-\gamma+\alpha\left[y_{0}-\left(-\gamma-b \widetilde{r}_{i, 0}\right)\right]$ plus

$$
\min _{\delta^{\prime} \in[-\delta, \delta]} \min _{\phi\left(p_{t}-\widetilde{p}_{j, t}\right) \in\left[-\gamma_{p}, \gamma_{p}\right]} \alpha \delta^{\prime}\left[\phi\left(p_{1}-\widetilde{p}_{j, 1}\right)-\phi\left(p_{0}-\widetilde{p}_{j, 0}\right)\right]=-2 \alpha \delta \gamma_{p}
$$

Note that $v^{*}\left(\varepsilon^{0}, s_{1}, p_{i, 1}^{*}\right)$ does not have a kink in the $p_{1}$ space. Approximate around $p_{1}=\widetilde{p}_{j, 1}$ to obtain a derivative is:

$$
\frac{d \ln v^{*}\left(\varepsilon^{0}, s_{1}, p_{i, 1}^{*}\right)}{d p_{1}}=-\frac{e^{\widetilde{r}_{i, 0}}}{e^{\widetilde{r}_{i, 0}}-e^{m y_{1}}}+b
$$

The constant term is given by evaluating $\ln v^{*}\left(\varepsilon^{0}, s_{1}, p_{i, 1}^{*}\right)$ at $p_{1}=\widetilde{p}_{j, 1}$, as:

$$
\ln \left(e^{\widetilde{r}_{i, 0}}-e^{y_{1}-\omega_{i, 1}}\right)+c_{t}-b \widetilde{r}_{i, 0}-\gamma+\alpha\left[y_{0}-\left(-\gamma-b \widetilde{r}_{i, 0}\right)\right]-2 \alpha \delta \gamma_{p}
$$

We now compute the difference $\ln v^{*}\left(\varepsilon^{0}, s_{1}, p_{i, 1}^{\text {index }}\right)-\ln v^{*}\left(\varepsilon^{0}, s_{1}, p_{i, 1}^{*}\right)$, up to their first-order approximation:

$$
\left(\frac{e^{\widetilde{r}_{i, 0}}}{e^{\widetilde{r}_{i, 0}}-e^{y_{1}-\omega_{i, 1}}}-b-\alpha \delta^{i n d e x}\right)\left(p_{1}-\widetilde{p}_{j, 1}\right)<0
$$

using the worst-case demand shape $\delta^{\text {index }}=\delta \operatorname{sgn}\left(p_{1}-\widetilde{p}_{j, 1}\right)$ and Proposition A2. The latter shows that the condition for having the optimal price $\widetilde{r}_{i, 1}$ be at the kink $\widetilde{r}_{i, 0}$ is that the derivatives at the right, based on demand elasticity $-b-\delta$, and at the left, using the elasticity $-b+\delta$, are negative and, respectively, positive.

\section{A.6 Dispersion of forecasts}

Here we detail how we use empirical evidence from Gaur et al. (2007) on survey data to evaluate the size of our calibrated ambiguity parameter $\gamma$. Gaur et al. (2007) use item-level forecasts of demand data from a skiwear manufacturer, called the Sport Obermeyer dataset. The dataset contains style-color level forecasts for 248 short lifecycle items for a selling season of about three months. The forecasts are done by members of a committee specifically constituted to forecast demand, consisting of: the president, a vice president, two designers, and the managers of marketing, production, and customer service. Raman et al. (2001) provides details on the forecasting procedures and on the dataset. 
Our model connects to the data in Gaur et al. (2007) as follows. They observe forecasts made prior to the product being introduced. Their statistic for the dispersion of these forecasts is reported as a coefficient of variation. Our model relates to this measure through the set of multiple priors. Indeed, in our model, prior to observing any realized demand signals, the firm entertains a set of forecasts about quantity sold. We connect this set to the dispersion of forecasts made by the committee described above. In particular, in our model the firm entertains the following time-zero set of forecasts on the level of demand

$$
\left[\exp \left(-\gamma-b p+0.5 \sigma_{z}^{2}\right), \exp \left(\gamma-b p+0.5 \sigma_{z}^{2}\right)\right]
$$

While in the data the set consists of only seven forecasters, we have a continuum. But we can compute the coefficient of variation $(\mathrm{CV})$ of these forecasts and compare it against the reported statistic. In particular, using a uniform distribution over the forecasts in the set above, the CV, normalized by the average forecast, equals

$$
C V=\frac{1}{\sqrt{3}} \frac{e^{\gamma}-e^{-\gamma}}{\left(e^{\gamma}+e^{-\gamma}\right)}
$$

Gaur et al. (2007) report in their Table 4 that the average level of coefficient of variation, scaled by the average forecast, across the products in the dataset equals $37.6 \%$. Plugging in the calibrated value of our ambiguity parameter $\gamma=0.614$, we obtain a CV equal to $31.58 \%$.

\section{A.7 Empirical link between aggregate and industry prices}

In this section, we use US CPI data to show that the relationship between aggregate and industry prices is time-varying and unstable over short-horizons. In particular, an econometrician would generally have very little confidence that short-run aggregate inflation is related to industry-level inflation, even though he can be confident that the two are cointegrated in the long-run. Thus, our assumption on the uncertainty over $\phi($.$) puts the firm on an equal footing with an econometrician$ outside of the model.

Our analysis uses the Bureau of Labor Statistics' most disaggregated 130 CPI indices as well as aggregate CPI inflation. The empirical exercise consists of the following regression method. For a specific industry $j$, we define its inflation rate between $t-k$ and $t$ as $\pi_{j, t, k}$ and similarly $\pi_{t, k}^{a}$ for aggregate CPI inflation. For each industry $j$, we run the rolling regressions:

$$
\pi_{j, t, k}=\beta_{j, k, t} \pi_{t, k}^{a}+u_{t}
$$

over three-year windows starting in 1995 and ending in 2010, and note that results are very similar if we use windows of 2 or 5 years instead. We repeat this exercise for $k$ equal to 1, 3, 6, 12 and 24 months. Finally, for each of these horizons we compute the fraction of regression coefficients $\beta_{j, k, t}$ 
(across industries and 3-year regression windows) that are statistically different from zero at the $95 \%$ level.

We find that for 1-month inflation rates, only $11.4 \%$ of the relationships between sectoral and aggregate inflation are statistically significant. For longer horizons $k$, these fractions generally remain weak but do rise over time: $26.4 \%, 40.6 \%, 58.5 \%$ and $69.1 \%$ for the $3-$, 6-, $12-$ and $24-$ month horizons respectively. This supports our assumption that while disaggregate and aggregate price indices might be cointegrated in the long run, their short-run relationship is weak.

In fact, not only is the relationship statistically weak in general, but it is highly unstable. This can be seen in Figure A.1, which shows the evolution of the coefficient $\beta_{j, k, t}$ for $k=3$ for 3 -yearwindow regressions starting in each month between 1995 and 2010, for four industries. Not only are there large fluctuations in the value of this coefficient over our sample, but sign reversals are common. In general, at any given date, there is little confidence that the near-future short-horizon industry-level inflation would be highly correlated with aggregate inflation, even though the data is quite clear that the two are tightly linked over the long-run.
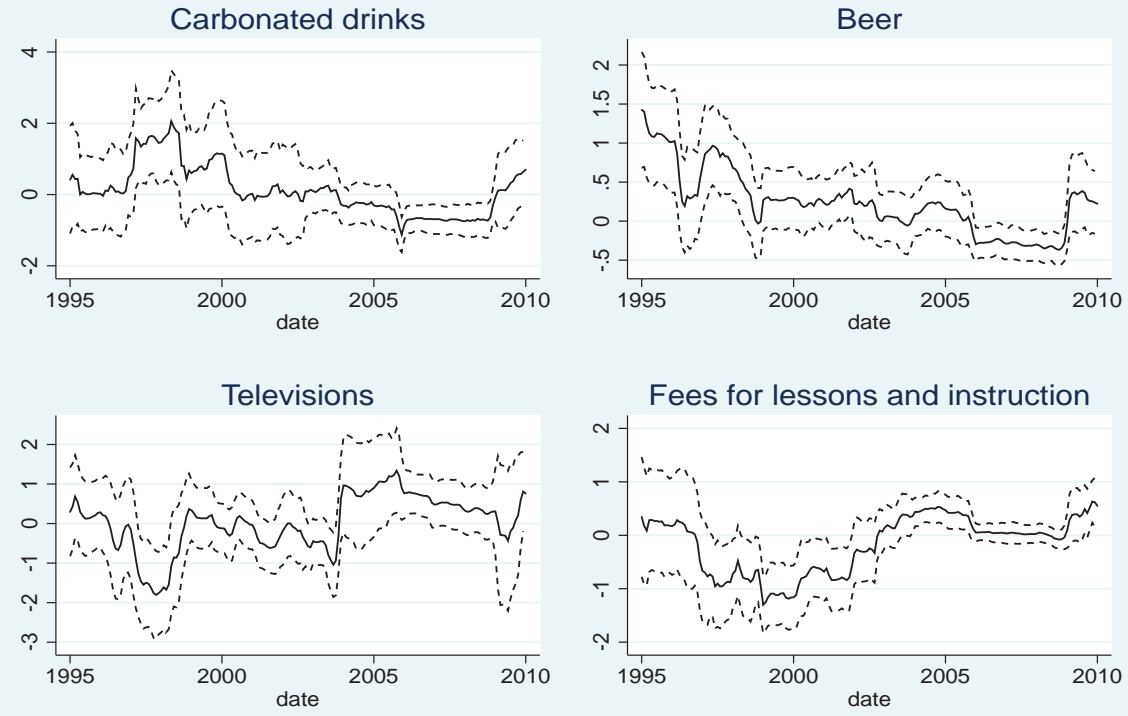

Figure A.1. 3-year rolling regressions of 3-month industry inflation on 3-month aggregate inflation for four categories. The solid line plots the point estimate of regression coefficient on aggregate inflation. The dotted lines plot the $95 \%$ confidence intervals.

\section{A.8 The typical information set at the stochastic steady state}

In this section we analyze in more depth the typical information set at the stochastic steady state. In the model, the price histories and demand realizations differ across firms. One reason is the idiosyncratic noise in demand realizations, but more importantly, the position of the demand signals is endogenous, because it depends on the past pricing decisions of the firm. With 
idiosyncratic productivity shocks, firms take different pricing decisions, and thus their information sets evolve differently. Let

$$
\mathcal{I}_{i t}=\left[\tilde{\mathbf{r}}_{i t}^{u n i q}, \mathbf{N}_{i t}, \hat{\mathbf{y}}_{i t}\right]
$$

be the 3-column matrix that characterizes the information set of firm $i$ at time $t$, where $\tilde{\mathbf{r}}_{i t}^{u n i q}$ is the vector of unique unambiguously estimated relative price points in the history of past price decisions, $\tilde{r}_{i}^{t}$, of firm $i$; $\mathbf{N}_{i t}$ is the associated vector of the number of times each of those unique price points has been chosen in the past; and $\hat{\mathbf{y}}_{i t}$ is the average, demeaned demand realization that the firm has seen at those unique price points. So each row of $\tilde{\mathbf{r}}_{i t}^{u n i q}$ is one of the unique price levels the firm has posted in the past, the corresponding row of $\mathbf{N}_{i t}$ is the number of times this price has been seen in the past, and the corresponding row of $\hat{\mathbf{y}}_{i t}$ is the average demeaned demand realizations the firm has experienced when choosing that price. The matrix $\mathcal{I}_{i t}$ fully described the information set of the firm, and is the sufficient statistic needed to compute the worst-case expected demand $\hat{x}_{i t}(\tilde{r})$.

The most striking characteristic of $\mathcal{I}_{i t}$ is that the average cardinality of $\tilde{\mathbf{r}}_{i t}^{\text {uniq }}$ is just six. Thus even though the average life span of firms in our model is 133 periods, the histories contain only 6 unique estimated relative prices on average. Another interesting characteristic, is that the average firm has not seen each of those six price points equally often, but in fact the most often posted price accounts for $74 \%$ of all observations, on average. Moreover, the second most often chosen price accounts for another $19 \%$ of all observations.

These features of the typical information set can be helpful in understanding the pricing moments the model generates. First, the sparse nature of the information set implies that the typical firm faces substantial amount of residual demand uncertainty even in the long run. The reason behind this substantial residual demand uncertainty is that the history of observations is endogenously sparse. In particular, the optimal policy leads the firm to often repeat estimated relative prices, resulting in a history of observations that provides a lot of information about the average level of demand at those select prices, but leaves the firm uncertain about the shape of its demand in between the observed price points. Hence our mechanism, which operates specifically through the uncertainty about the local shape of demand, has a strong bite even at the steady state of the model, when firms have seen long histories of demand observations. In fact, because of the local nature of learning and the endogenous location of demand signals, learning proceeds so slowly that the mechanism survives even if firms live for thousands of periods. We explore this implication further in Online Appendix B.6 (on the authors' website) by setting $\lambda_{\phi}=0$. In the same appendix we also show that the accumulation of new information could in fact change the optimal position of some of the reference prices.

Second, the typical information set still contains multiple distinct price points at which the firm has reduced demand uncertainty. Thus, the optimal pricing action does not only lead to price stickiness (i.e. reluctance to leave one of the price points with low residual demand uncertainty), 
but also to significant memory in prices since conditional on a move, the firm is likely to move to one of the other price points it has learned about in the past, rather than to a brand new price.

Lastly, the typical information set also tends to feature one "dominant" price at which the firm has accumulated most of its past signals. This gives rise to pricing patterns where the firm has a clear "modal" or "reference" price point that it tends to stay at for prolonged periods of time and return to often.

\section{A.9 Cell-based evidence on hazard functions}
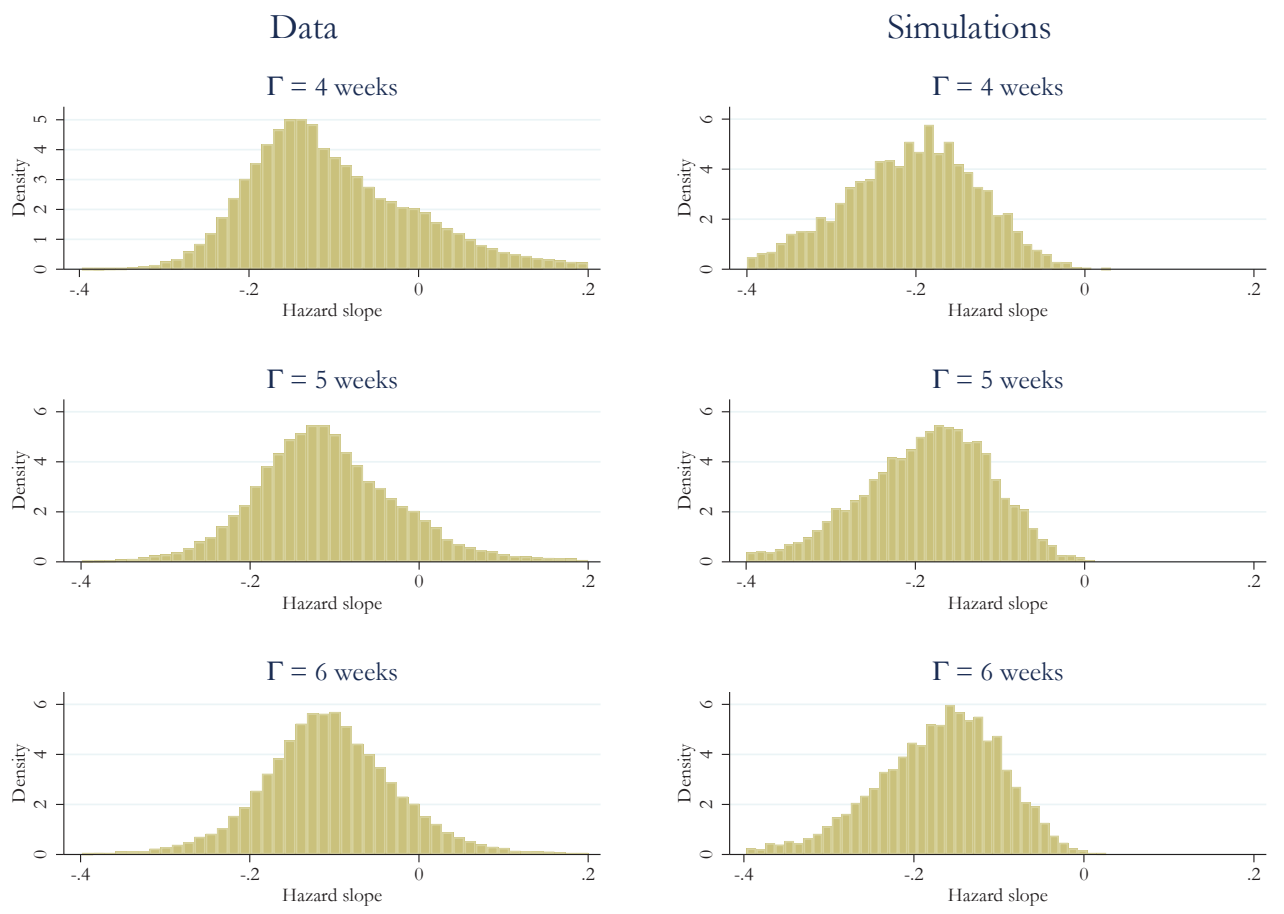

Figure A.2. Distributions of the cell-based hazard slopes. A slope is defined as the difference between the price change frequencies of old $(\tau \geq \Gamma)$ and young $(\tau<\Gamma)$ prices. Empirical (left) and simulated (right) distributions.

In Figure A.2, we plot the distributions of cell-based slopes obtained using the approach of Campbell and Eden (2014). A cell is a specific product sold in a given store, while the slope is computed as the difference between the price change frequencies of older and younger prices. An "old" price is one that has survived at least $\Gamma$ weeks. In order to obtain a more complete comparison between the data and the model simulations than just the average slope, we plot both the empirical (left column) and simulated (right) distributions of the cell-based hazard slopes, for $\Gamma=4,5,6$. 